\title{
Points of infinite multiplicity of planar Brownian motion: measures and local times
}

\author{
by
}

Elie Aïdékon, Yueyun Hu, and Zhan Shi

\begin{abstract}
Summary. It is well-known (see Dvoretzky, Erdős and Kakutani [7] and Le Gall [13]) that a planar Brownian motion $\left(B_{t}\right)_{t \geq 0}$ has points of infinite multiplicity, and these points form a dense set on the range. Our main result is the construction of a family of random measures, denoted by $\left\{\mathcal{M}_{\infty}^{\alpha}\right\}_{0<\alpha<2}$, that are supported by the set of the points of infinite multiplicity. We prove that for any $\alpha \in(0,2)$, almost surely the Hausdorff dimension of $\mathcal{M}_{\infty}^{\alpha}$ equals $2-\alpha$, and $\mathcal{M}_{\infty}^{\alpha}$ is supported by the set of thick points defined in Bass, Burdzy and Khoshnevisan [1] as well as by that defined in Dembo, Peres, Rosen and Zeitouni [5].
\end{abstract}

Keywords. Planar Brownian motion, infinite multiplicity, thick points.

2010 Mathematics Subject Classification. 60J65.

\section{Introduction}

\subsection{Problem and results}

It is well-known that a planar Brownian motion has points of infinite multiplicity, and these points form a dense set on the range (Dvoretzky, Erdős and Kakutani [7] and Le Gall [13]); see Le Gall ([14], p. 204) for comments on the proof.

In this paper, we restrict our attention to the thick points which constitute a dense subset of points of infinite multiplicity. In the literature, there are two important ways to define thick points, one by Bass, Burdzy and Khoshnevisan [1] through the number of crossings and another one by Dembo, Peres, Rosen and Zeitouni [5] through the occupation times.

LPSM, Sorbonne Université Paris VI \& IUF, 4 place Jussieu, F-75252 Paris Cedex 05, France, elie.aidekon@upmc.fr LAGA, Université Paris XIII, 99 avenue J-B Clément, F-93430 Villetaneuse, France, yueyun@math.univ-paris13.fr LPSM, Sorbonne Université Paris VI, 4 place Jussieu, F-75252 Paris Cedex 05, France, zhan.shi@upmc.fr 
Let $\left(B_{t}\right)_{t \geq 0}$ be the standard planar Brownian motion started at 0 defined on a complete probability space $\left(\Omega, \mathscr{F},\left(\mathscr{F}_{t}\right)_{t \geq 0}, \mathbb{P}\right)$, where $\mathscr{F}_{t}:=\sigma\left\{B_{s}, s \in[0, t]\right\}, t \geq 0$, is the natural filtration of $B$. For any $x \in \mathbb{R}^{2}$ and $r>0$, denote by $\mathcal{C}(x, r)$ (resp: $\mathcal{B}(x, r)$ ) the circle (resp: open disc) centered at $x$ and with radius $r$. Let $N_{x}(r)$ be the number of crossings from $x$ to $\mathcal{C}(x, r)$ by $\left(B_{t}\right)$ till $T_{\mathcal{C}(0,1)}$, where $T_{\mathcal{C}(0,1)}:=\inf \left\{t \geq 0: B_{t} \in \mathcal{C}(0,1)\right\}$ is the first hitting time of the unit circle $\mathcal{C}(0,1)$. Fix $\alpha \in(0,2)$. Bass, Burdzy and Khoshnevisan [1] studied the following set:

$$
\mathcal{A}_{\alpha}:=\left\{x \in \mathcal{B}(0,1): \lim _{r \rightarrow 0^{+}} \frac{N_{x}(r)}{\log 1 / r}=\alpha\right\} .
$$

For any Borel measure $\beta$ on $\mathbb{R}^{2}$, let $\operatorname{Dim}(\beta)$ be the Hausdorff dimension of $\beta$ :

$$
\operatorname{Dim}(\beta):=\inf \left\{r: \exists \text { Borel set } A \text { such that } \beta\left(A^{c}\right)=0 \text { and } \operatorname{dim}_{\mathrm{H}}(A)=r\right\},
$$

where $\operatorname{dim}_{\mathrm{H}}(A)$ denotes the Hausdorff dimension of the set $A$. The main result in [1] can be stated as follows:

Theorem A (Bass, Burdzy and Khoshnevisan [1]). Let $\alpha \in\left(0, \frac{1}{2}\right)$. Almost surely there exists a measure $\beta_{\alpha}$ carried by $\mathcal{A}_{\alpha}$. Moreover,

$$
\operatorname{Dim}\left(\beta_{\alpha}\right)=2-\alpha, \quad \text { a.s. }
$$

The random measure $\beta_{\alpha}$ in Theorem A gives important information on the set of points to which planar Brownian motion makes "many visits". For example, it plays a crucial role in Cammarota and Mörters [4] who gave a characterization of the gauge functions for the level sets of planar Brownian motion, confirming a conjecture of Taylor [21].

Another definition of thick points was given by Dembo, Peres, Rosen and Zeitouni [5]: A point $x \in \mathbb{R}^{2}$ is called an $\alpha$-thick point (called perfectly thick point in [5]) if

$$
\lim _{r \rightarrow 0^{+}} \frac{1}{r^{2}(\log r)^{2}} \int_{0}^{T_{\mathcal{C}(0,1)}} 1_{\left\{B_{s} \in \mathcal{B}(x, r)\right\}} \mathrm{d} s=\alpha,
$$

where, as pointed out in [5], $T_{\mathcal{C}(0,1)}$ can be replaced by any positive and finite $\left(\mathscr{F}_{t}\right)$-stopping time.

The fractal measure of the $\alpha$-thick points was studied in depth in [5]; in particular the following result holds:

Theorem B (Dembo, Peres, Rosen and Zeitouni [5]) For $0 \leq \alpha \leq 2$,

$$
\operatorname{dim}_{\mathrm{H}}\{\alpha \text {-thick points }\}=2-\alpha, \quad \text { a.s. }
$$


To the best of our best knowledge, it is still an open question whether the two ways of defining thick points are equivalent or not. For instance, we have not been able to determine the Hausdorff dimension of $\mathcal{A}_{\alpha}$.

The starting point of our study is to generalize Theorem A to all parameters $\alpha \in(0,2)$. Our construction of random measures, different from that in [1] where the authors utilized the local times on circles, relies on a change of measures involving the excursions around points with infinite multiplicity. This change of measures is given by [1], Theorem 5.2, where it plays a crucial role.

We consider Brownian motion inside a domain. By a domain in $\mathbb{R}^{2}$ we mean an open, connected and bounded subset of $\mathbb{R}^{2}$. Given a domain $D$, a boundary point $z \in \partial D$ is said to be nice if there exists a one-to-one analytic function $f$ from the unit disc such that $f(0)=z$ and the image of the set of points of the unit disc with positive imaginary part is the intersection of $D$ with the image of the unit disc by $f$. A domain $D$ is nice if every boundary point, except perhaps a finite number of them, is nice (in Lawler [10], p.48, $\partial D$ is said to be piecewise analytic). Let $\bar{D}:=D \cup \partial D$. We say that a point $z \in \bar{D}$ is nice if either $z \in D$ or $z$ is a nice boundary point.

Denote by $\mathbb{P}_{D}^{z, z^{\prime}}$ the probability law of a Brownian excursion inside $D$ from $z$ to $z^{\prime}$, whose definition is given in Section 2.1.

The main result of this paper reads as follows:

Theorem 1.1 Let $D$ be a simply connected nice domain. Let $z$ and $z^{\prime}$ be distinct nice points of $\bar{D}$. Fix $\alpha \in(0,2)$. With $\mathbb{P}_{D}^{z, z^{\prime}}$-probability one, there exists a random finite measure $\mathcal{M}_{\infty}^{\alpha}$ carried by $\mathcal{A}_{\alpha}$ as well as by the set of $\alpha$-thick points; moreover,

$$
\operatorname{Dim}\left(\mathcal{M}_{\infty}^{\alpha}\right)=2-\alpha, \quad \mathbb{P}_{D}^{z, z^{\prime}} \text {-a.s. }
$$

We mention that $\mathcal{M}_{\infty}^{\alpha}$ is uniquely determined by the forthcoming formula (5.1), the latter being analogous to [1], Theorem 5.2. Since $\mathcal{M}_{\infty}^{\alpha}$ is supported by the set of $\alpha$-thick points, (1.3) yields that almost surely, $\operatorname{dim}_{H}\{\alpha$-thick points $\} \geq 2-\alpha$, giving a new proof of the lower bound in Theorem B.

We will see that equation (5.1) also yields the existence, ${ }^{4}$ for $\mathcal{M}_{\infty}^{\alpha}(\mathrm{d} x)$-almost every $x$, of a continuous and non-decreasing additive functional $\left(\mathfrak{L}_{t}^{x}\right)_{t \geq 0}$, called local times at the point $x$. Define

$$
T_{x}:=\inf \left\{t>0: B_{t}=x\right\}, \quad x \in \mathbb{R}^{2} .
$$

\footnotetext{
${ }^{4}$ This was a private question by Chris Burdzy.
} 
Theorem 1.2 Let $D$ be a simply connected nice domain. Let $z$ and $z^{\prime}$ be distinct nice points of $\bar{D}$. Fix $\alpha \in(0,2)$. With $\mathbb{P}_{D}^{z, z^{\prime}}$-probability one, $\mathcal{M}_{\infty}^{\alpha}(\mathrm{d} x)$-almost everywhere, there exists a continuous Brownian additive functional $\left(\mathfrak{L}_{t}^{x}\right)_{t \geq 0}$ such that

$$
\mathfrak{L}_{t}^{x}=\lim _{r \rightarrow 0^{+}} \frac{1}{r^{2}(\log r)^{2}} \int_{0}^{t} 1_{\left\{\left|B_{s}-x\right|<r\right\}} \mathrm{d} s, \quad \forall t \geq 0 ;
$$

moreover the support of $\mathrm{d} \mathfrak{L}_{t}^{x}$ is identified as the level set $\left\{t \geq 0: B_{t}=x\right\}$.

In Theorem 1.2, under $\mathbb{P}_{D}^{z, z^{\prime}}$, the process is killed upon first hitting $z^{\prime}$, i.e., at time $T_{z^{\prime}}:=\inf \left\{t>0: B_{t}=z^{\prime}\right\}$. So the local time $\mathfrak{L}_{t}^{x}$ is defined for $t \in\left[0, T_{z^{\prime}}\right]$. We are going to see that $\mathfrak{L}_{T_{z^{\prime}}}^{x}=\alpha$ (with $\mathbb{P}_{D}^{z, z^{\prime}}$-probability one, $\mathcal{M}_{\infty}^{\alpha}(\mathrm{d} x)$-almost everywhere).

\subsection{A brief description of the construction of $\mathcal{M}_{\infty}^{\alpha}$}

Let $D$ be a nice domain, and let $x \in D$ and $z, z^{\prime}$ distinct nice points of $\bar{D}$, different from $x$. Let as before $T_{x}:=\inf \left\{t>0: B_{t}=x\right\}$. We consider a probability measure $\mathbb{Q}_{x, D}^{z, z^{\prime}, \alpha}$ similar to the measure $\mathbf{Q}_{\alpha}^{x}$ introduced in Bass, Burdzy and Khoshnevisan ([1], p. 606): Under $\mathbb{Q}_{x, D}^{z, z^{\prime}, \alpha},\left(B_{t}\right)_{t \geq 0}$ is split into three parts:

1. Until time $T_{x}, B$ is a Brownian motion starting from $z$ and conditioned at hitting $x$ before $\partial D$, whose law $\mathbb{P}_{D}^{z, x}$ is defined in Notation 2.1 .

2. After $T_{x}$, the trajectory is a concatenation of Brownian loops generated by a Poisson point process $\left(\mathfrak{e}_{s}\right)_{s \geq 0}$ with intensity $1_{[0, \alpha]} \mathrm{d} t$ in time and $\nu_{D}(x, x)$ in space, where $\nu_{D}(x, x)$ denotes the law of Brownian loops in $D$ at $x$ (see (2.12) for the definition). ${ }^{5}$

3. The last part of the trajectory is a standard Brownian motion in $D$, started from $x$ and conditioned to hit $z^{\prime}$ if $z^{\prime} \in D$ or to exit $D$ at $z^{\prime}$ if $z^{\prime} \in \partial D$. The law $\mathbb{P}_{D}^{x, z^{\prime}}$ of this process is defined in $(2.7)$.

Consider $D_{1} \subset D$ a nice domain containing $x$. The baseline in our construction of $\mathcal{M}_{\infty}^{\alpha}$, stated as Corollary 3.6 , is the absolute continuity of $\mathbb{Q}_{x, D}^{z, z^{\prime}, \alpha}$ with respect to $\mathbb{P}_{D}^{z, z^{\prime}}$ considered at $\mathscr{F}_{D_{1}}^{+}$, where $\mathscr{F}_{D_{1}}^{+}$denotes the sigma-algebra generated by the excursions outside $D_{1}$ together with the order of their appearances (see Notation 3.4). Denote

\footnotetext{
${ }^{5}$ When $\alpha=0$, the second part is reduced to the single point $\{x\}$.

Let us say a few words on the concatenation of the loops $\left(\mathfrak{e}_{s}\right)$. Denote by $\zeta(\mathfrak{e})$ the lifetime of a loop $\mathfrak{e}$. Remark that $\sum_{s<\alpha} \zeta\left(\mathfrak{e}_{s}\right)<\infty$, a.s., see (6.2) for the law of $\zeta$. For $T_{x}<t \leq T_{x}+\sum_{s<\alpha} \zeta\left(\mathfrak{e}_{s}\right)$, let $s \in(0, \alpha]$ be such that $t-T_{x} \in\left[\sum_{u<s} \zeta\left(\mathfrak{e}_{u}\right), \sum_{u \leq s} \zeta\left(\mathfrak{e}_{u}\right)\right)$. We define $B_{t}:=\mathfrak{e}_{s}\left(t-T_{x}-\sum_{u<s} \zeta\left(\mathfrak{e}_{u}\right)\right)$.
} 
by $M_{D_{1}}(x, \alpha)$ the Radon-Nikodym derivative (up to a renormalization factor $M_{D}(x, \alpha)$ ). Then $M_{D_{1}}(x, \alpha)$ satisfies a certain restriction property (see Corollary 3.7).

Now we construct $\mathcal{M}_{\infty}^{\alpha}$ as follows: Let $D$ be a simply connected nice domain and let $\mathscr{D}_{n}$ be the connected components of $D$ minus a grid of mesh size $2^{-n}$. By using Corollary 3.7, we may construct a sequence of random measures $\mathcal{M}_{\mathscr{D}_{n}}^{\alpha}$ (defined in (4.1)). The measure $\mathcal{M}_{\infty}^{\alpha}$ is nothing but the (nonnegative martingale) limit of $\mathcal{M}_{\mathscr{D}_{n}}^{\alpha}$ as $n \rightarrow \infty$, and the limit is not trivial and defines a (finite) measure thanks to the uniform integrability of $\left(\mathcal{M}_{\mathscr{D}_{n}}^{\alpha}\right)_{n \geq 1}$ (see Theorem 4.1). This gives the construction of $\mathcal{M}_{\infty}^{\alpha}$.

\subsection{Comparison with previous works}

In Dembo, Peres, Rosen and Zeitouni [5], the main interest was focused on the size of the set of the thick points; as a tool, the authors constructed a measure on the thick points as a limit along subsequences of a tight family of measures, for all $\alpha \in(0,2)$. In Bass, Burdzy and Khoshnevisan [1], a measure (in fact, the measure $\beta_{\alpha}$ recalled in Theorem A) on the thick points was constructed by means of an $L^{2}$-limit, for all $\alpha \in\left(0, \frac{1}{2}\right)$; a formula characterizing the measure $\beta_{\alpha}$ will be recalled in our paper (see Proposition 5.1).

Our work makes a link between [1] and [5]. Inspired by the conceptual approach of Lyons [15] and Lyons, Pemantle and Peres [16] for branching processes, we extend the measure $\beta_{\alpha}$ in [1] to all $\alpha \in(0,2)$, relying on the martingale structure instead of computations of moments. It is to the measure $\beta_{\alpha}$ in a similar spirit as Lyons [15] to the Biggins martingale convergence theorem for spatial branching processes originally established by Biggins [2].

After this work was completed, a paper of Jego [8] managed to construct the measure $\beta_{\alpha}$ for all $\alpha \in(0,2)$ by approximating it via the thick points, as in [1], relying on a truncated second moment method.

We close this introduction by describing the organization of this paper:

- Section 2: We collect some results on the Brownian measure $\mathbb{P}_{D}^{z, z^{\prime}}$ on the paths inside a nice domain $D$ from $z$ to $z^{\prime}$ and on the $\sigma$-finite measure $\nu_{D}(x, x)$ on the Brownian loops at $x$.

- Section 3: We characterize the law of the macroscopic excursions at $x$ under $\mathbb{Q}_{x, D}^{z}$ (Proposition 3.2) and get Corollary 3.6. We also establish Proposition 3.9 which plays a key role in the proof of Theorem 4.1. 
- Section 4: A standard argument says that the proof of Theorem 4.1 boils down to the study of $\mathcal{M}_{\infty}^{\alpha}$ under $\mathbb{Q}_{x, D}^{z}$ (Proposition 4.2). The proof of Proposition 4.2 is based on a truncation argument in the computation of moments under $\mathbb{Q}_{x, D}^{z}$, which also provides the needed capacity estimates (see (4.18)) in the proof of (1.3).

- Section 5: The measure $\mathcal{M}_{\infty}^{\alpha}$ satisfies a certain conformal invariance, up to a multiplicative factor, which implies its almost sure positivity.

- Section 6: We prove that $\mathcal{M}_{\infty}^{\alpha}$ is supported by $\mathcal{A}_{\alpha}$ as well as by the set of $\alpha$-thick points (Corollary 6.1 and Theorem 6.2) and complete the proof of Theorem 1.1. The proof of Theorem 1.2 is also given in Section 6 .

- Section 7: We identify the measure $\mathcal{M}_{\infty}^{\alpha}$ in the case $\alpha=0$ and discuss its relationship with the intersection local times of independent Brownian motions.

For notational convenience, we write $\mathbb{E}[X, A]:=\mathbb{E}\left[X 1_{A}\right]$ when $X$ is a random variable and $A$ is an event (and we write $\mathbb{E}\left[X, A_{1}, A_{2}\right]$ for $\mathbb{E}[X, A]$ if $A=A_{1} \cap A_{2}$ ). When $\nu$ is a positive measure, we write $\nu(X):=\int X \mathrm{~d} \nu$ and use the similar notation $\nu(X, A)$ for $\int_{A} X \mathrm{~d} \nu$. Finally, by $f(x) \sim g(x)$ as $x \rightarrow x_{0}$ we mean that $\lim _{x \rightarrow x_{0}} \frac{f(x)}{g(x)}=1$.

\section{Preliminaries on Brownian excursions}

At first we recall some facts, taken from Lawler ([10], Chapter 2), on the Green function and the Poisson kernel. Let $D$ be a nice domain and consider $x \in D$. The harmonic measure $\mathbb{P}^{x}\left(B_{T_{\partial D}} \in \bullet\right)$ is absolutely continuous with respect to the one-dimensional Lebesgue measure, whose density is called the Poisson kernel and is denoted by $H_{D}(x, y), y \in \partial D$. For any nice point $y \in \partial D, H_{D}(\bullet, y)$ is harmonic in $D$.

Let $p_{D}(t, x, y), x \in D, y \in D$ be the density (in $y$ ) of the transition probabilities of $B_{t \wedge T_{D^{c}}}$ under $\mathbb{P}^{x}$. We define the Green function $G_{D}$ by

$$
G_{D}(x, y):=\pi \int_{0}^{\infty} p_{D}(t, x, y) \mathrm{d} t, \quad x, y \in D .
$$

Then

$$
G_{D}(x, y)=\log \frac{1}{|x-y|}+O(1), \quad y \rightarrow x .
$$

For any nice point $y \in \partial D$, denote by $\mathbf{n}_{y}$ the inward normal at $y$. We have (see Lawler $[10]$, p. 55) that

$$
\lim _{\varepsilon \rightarrow 0^{+}} \frac{1}{\varepsilon} G_{D}\left(x, y+\varepsilon \mathbf{n}_{y}\right)=2 \pi H_{D}(x, y)
$$


Following Lawler and Werner [11] and Lawler [10], Section 5.2, we introduce the boundary Poisson kernel: for $y^{\prime} \neq y$ distinct nice points of $\partial D$, define

$$
H_{D}\left(y, y^{\prime}\right):=\lim _{\varepsilon \rightarrow 0^{+}} \frac{1}{\varepsilon} H_{D}\left(y+\varepsilon \mathbf{n}_{y}, y^{\prime}\right),
$$

For sake of concision, we will use the following notation: if $z, z^{\prime}$ are distinct points of $D$, we define

$$
H_{D}\left(z, z^{\prime}\right):=\frac{1}{2 \pi} G_{D}\left(z, z^{\prime}\right) .
$$

Finally, we define $H_{D}\left(z, z^{\prime}\right):=H_{D}\left(z^{\prime}, z\right)$ when $z \in \partial D$ and $z^{\prime} \in D$. The function $H_{D}$ is symmetric and has the following property (see Lawler [10], Section 5.2): if $D, D^{\prime}$ are two nice domains, $z, z^{\prime}$ nice points of $\bar{D}$ (recall that by $y$ nice point of $\bar{D}$, we mean $y \in D$ or $y$ nice point of $\partial D), \Psi: D \rightarrow D^{\prime}$ a conformal transformation such that $\Psi(z)$ and $\Psi\left(z^{\prime}\right)$ are nice points of $\overline{D^{\prime}}$, then

- if $z, z^{\prime} \in D, H_{D^{\prime}}\left(\Psi(z), \Psi\left(z^{\prime}\right)\right)=H_{D}\left(z, z^{\prime}\right)$,

- if $z \in D$ and $z^{\prime} \in \partial D, H_{D^{\prime}}\left(\Psi(z), \Psi\left(z^{\prime}\right)\right)=\left|\Psi^{\prime}\left(z^{\prime}\right)\right|^{-1} H_{D}\left(z, z^{\prime}\right)$,

- if $z, z^{\prime} \in \partial D, H_{D^{\prime}}\left(\Psi(z), \Psi\left(z^{\prime}\right)\right)=\left|\Psi^{\prime}(z)\right|^{-1}\left|\Psi^{\prime}\left(z^{\prime}\right)\right|^{-1} H_{D}\left(z, z^{\prime}\right)$.

Beware that with this definition, $H_{D}$ is not continuous at the boundary of $D$. Indeed, from (2.2), we see that for $z \in D$ and $z^{\prime}$ nice point of $\partial D$,

$$
\lim _{\varepsilon \rightarrow 0^{+}} \frac{1}{\varepsilon} H_{D}\left(z, z^{\prime}+\varepsilon \mathbf{n}_{z^{\prime}}\right)=H_{D}\left(z, z^{\prime}\right) .
$$

With this notation, we may give a unified presentation on the forthcoming $h$-transform (2.7).

As in Lawler ([10], Chapter 5), we consider $\mathcal{K}$ the set of all parametrized continuous planar curves $\gamma$ defined on a finite time-interval $\left[0, t_{\gamma}\right]$ with $t_{\gamma} \in(0, \infty)$. For any closed subset $A \subset \mathbb{R}^{2}$, we call $\gamma$ an excursion away from $A$ if

$$
\gamma(0) \in A, \quad \gamma\left(t_{\gamma}\right) \in A, \quad \gamma(s) \notin A, \forall s \in\left(0, t_{\gamma}\right) .
$$

More generally, for any $0 \leq s<t<\infty$, we call $(\gamma(u), u \in[s, t])$ an excursion away from $A$ if $u \mapsto \gamma(u+s), u \in[0, t-s]$, is an excursion away from $A$.

The space $\mathcal{K}$ is endowed with the natural filtration of the canonical coordinate process $\left(\mathfrak{e}_{t}\right)_{t \geq 0}$. We denote by

$$
T_{A}:=\inf \left\{t>0: \mathfrak{e}_{t} \in A\right\},
$$


the first hitting time of a set $A$ by $\left(\mathfrak{e}_{t}\right)$. We will write $\left(B_{t}\right)$ in place of $\left(\mathfrak{e}_{t}\right)$ when the underlying measure is a probability measure (such as $\mathbb{P}_{D}^{z}$ and $\mathbb{P}_{D}^{z, z^{\prime}}$ ).

For any $0 \leq s<t<\infty$, we denote by $B_{[s, t]}$ the trajectory $u \in[0, t-s] \mapsto B_{u+s}$, an element in $\mathcal{K}$. We define $\mathfrak{e}_{[s, t]}$ in the same way.

\subsection{Excursion measures inside D}

Recall that for any Borel set $A \subset \mathbb{R}^{2}$,

$$
T_{A}:=\inf \left\{t>0: B_{t} \in A\right\}
$$

Let $D$ be a nice domain. For $x \in D$, denote by $\mathbb{P}_{D}^{x}$ the probability measure under which $\left(B_{t}, t \geq 0\right)$ is a Brownian motion starting from $x$ and killed at time $T_{\partial D}$. We introduce the probability measure $\mathbb{P}_{D}^{z, z^{\prime}}$ (which is the normalized excursion measure denoted by $\mu_{D}^{\#}\left(z, z^{\prime}\right)$ in Lawler [10], Section 5.2) as follows; the measure is supported by trajectories from $z$ to $z^{\prime}$ in $D$.

Notation 2.1 Let $D$ be a nice domain. Let $z$ and $z^{\prime}$ be distinct nice points of $\bar{D}$. We define the probability measure $\mathbb{P}_{D}^{z, z^{\prime}}$ as follows.

(i) If $z \in D$ and $z^{\prime} \in \bar{D}$, we let $\mathbb{P}_{D}^{z, z^{\prime}}$ be the law of the Brownian motion starting at $z$, conditioned to hit $z^{\prime}$ before $\partial D$ if $z^{\prime} \in D$, and conditioned to exit $D$ at $z^{\prime}$ if $z^{\prime} \in \partial D$. It is given by the h-transform of the Brownian motion: for any $0<r<\left|z-z^{\prime}\right|$,

$$
\left.\frac{\mathrm{d} \mathbb{P}_{D}^{z, z^{\prime}}}{\mathrm{d} \mathbb{P}_{D}^{z}}\right|_{\mathscr{F}_{T_{\mathcal{C}\left(z^{\prime}, r\right)}}}=\frac{H_{D}\left(B_{\left.T_{\mathcal{C}\left(z^{\prime}, r\right)}, z^{\prime}\right)}\right.}{H_{D}\left(z, z^{\prime}\right)} 1_{\left\{T_{\mathcal{C}\left(z^{\prime}, r\right)}<T_{\partial D}\right\}}
$$

(ii) If $z, z^{\prime} \in \partial D$, we define $\mathbb{P}_{D}^{z, z^{\prime}}$ as the limit of $\mathbb{P}_{D}^{y, z^{\prime}}$ as $y \rightarrow z$ and $y \in D$ in the sense of (2.8) below. It is the excursion measure at $z$ conditioned to exit $D$ at $z^{\prime}$.

(iii) If $z \in \partial D$ and $z^{\prime} \in D$, we define $\mathbb{P}_{D}^{z, z^{\prime}}$ as the limit of $\mathbb{P}_{D}^{y, z^{\prime}}$ as $y \rightarrow z$ and $y \in D$ in the sense of (2.8). It is the excursion measure at $z$ conditioned to hit the interior point $z^{\prime} \in D$.

For any $z \in \partial D$ nice point, the limit of $\mathbb{P}_{D}^{y, z^{\prime}}$ when $y \rightarrow z$ is understood in the following sense: for any $0<r<\left|z-z^{\prime}\right|$, any $\mathbf{A} \in \sigma\left\{B_{T_{D \cap \mathcal{C}(z, r)+t}}, t \geq 0\right\}$,

$$
\mathbb{P}_{D}^{z, z^{\prime}}(\mathbf{A})=\lim _{\varepsilon \rightarrow 0^{+}} \mathbb{P}_{D}^{z+\varepsilon \mathbf{n}_{z}, z^{\prime}}(\mathbf{A})
$$

See Appendix A.1 for a justification of (2.8). 
There is a time-reversal relationship between $\mathbb{P}_{D}^{z, z^{\prime}}$ and $\mathbb{P}_{D}^{z^{\prime}, z}$ (see Lawler [10], Section $5.2)$ which can also be checked by applying the general theory on the time-reversal of Markov processes at cooptional times (see Revuz and Yor [20], Theorem VII.4.5): for any $z, z^{\prime}$ distinct nice points of $\bar{D}$,

$$
\left(\left(B_{T_{z^{\prime}}-t}, 0 \leq t \leq T_{z^{\prime}}\right) \text { under } \mathbb{P}_{D}^{z, z^{\prime}}\right) \stackrel{\text { (law) }}{=}\left(\left(B_{t}, 0 \leq t \leq T_{z}\right) \text { under } \mathbb{P}_{D}^{z^{\prime}, z}\right)
$$

If $D, D^{\prime}$ are two nice domains, $z, z^{\prime}$ nice points of $\bar{D}, \Psi: D \rightarrow D^{\prime}$ a conformal transformation such that $\Psi(z)$ and $\Psi\left(z^{\prime}\right)$ are nice points of $\overline{D^{\prime}}$, then the image measure of $\mathbb{P}_{D}^{z, z^{\prime}}$ by $\Psi$ is $\mathbb{P}_{D^{\prime}}^{\Psi(z), \Psi\left(z^{\prime}\right)}$.

We introduce the following notation: for $D_{1} \subset D$ two nice domains, define

$$
\begin{aligned}
\mathcal{J}\left(D, D_{1}\right):= & \left\{z \in \partial D: \text { nice point such that } z \in \partial D_{1}\right. \\
& \text { and } \left.\exists r>0 \text { such that } D \cap \mathcal{B}(z, r)=D_{1} \cap \mathcal{B}(z, r)\right\} .
\end{aligned}
$$

Lemma 2.2 Let $D_{1} \subset D$ be two nice domains.

(i) For $z^{\prime}$ nice point of $\bar{D}$ and $z \in D_{1}$ with $z \neq z^{\prime}$, for any nice point $y \in \partial D_{1} \backslash \partial D$,

$$
\mathbb{P}_{D}^{z, z^{\prime}}\left(T_{z^{\prime}}>T_{\partial D_{1}}, B_{T_{\partial D_{1}}} \in \mathrm{d} y\right)=\frac{H_{D}\left(y, z^{\prime}\right)}{H_{D}\left(z, z^{\prime}\right)} H_{D_{1}}(z, y) \mathrm{d} y .
$$

(ii) For $z, z^{\prime}$ distinct points of $D_{1} \cup \mathcal{J}\left(D, D_{1}\right)$, we have

$$
\mathbb{P}_{D}^{z, z^{\prime}}\left(T_{z^{\prime}} \leq T_{\partial D_{1}}\right)=\frac{H_{D_{1}}\left(z, z^{\prime}\right)}{H_{D}\left(z, z^{\prime}\right)} .
$$

Proof. (i) For any nonnegative measurable function $F$ on $\mathcal{K}$, we deduce from the monotone convergence theorem that

$$
\mathbb{E}_{D}^{z, z^{\prime}}\left(F\left(B_{\left[0, T_{\partial D_{1}}\right]}\right), T_{z^{\prime}}>T_{\partial D_{1}}\right)=\lim _{r \rightarrow 0^{+}} \mathbb{E}_{D}^{z, z^{\prime}}\left(F\left(B_{\left[0, T_{\partial D_{1}}\right]}\right), T_{\mathcal{C}\left(z^{\prime}, r\right)}>T_{\partial D_{1}}\right) .
$$

Applying the change of measures in (2.7) to $T_{\mathcal{C}\left(z^{\prime}, r\right)} \wedge T_{\partial D_{1}}$ in place of $T_{\mathcal{C}\left(z^{\prime}, r\right)}$, we have

$$
\begin{aligned}
& \mathbb{E}_{D}^{z, z^{\prime}}\left(F\left(B_{\left[0, T_{\partial D_{1}}\right]}\right), T_{\mathcal{C}\left(z^{\prime}, r\right)}>T_{\partial D_{1}}\right) \\
= & \frac{1}{H_{D}\left(z, z^{\prime}\right)} \mathbb{E}_{D}^{z}\left[H_{D}\left(B_{T_{\partial D_{1}}}, z^{\prime}\right) F\left(B_{\left[0, T_{\partial D_{1}}\right]}\right), T_{\partial D} \wedge T_{\mathcal{C}\left(z^{\prime}, r\right)}>T_{\partial D_{1}}\right] .
\end{aligned}
$$

Hence, taking the limit $r \rightarrow 0^{+}$,

$$
\begin{aligned}
& \mathbb{E}_{D}^{z, z^{\prime}}\left(F\left(B_{\left[0, T_{\partial D_{1}}\right]}\right), T_{z^{\prime}}>T_{\partial D_{1}}\right) \\
= & \frac{1}{H_{D}\left(z, z^{\prime}\right)} \mathbb{E}_{D}^{z}\left[H_{D}\left(B_{T_{\partial D_{1}}}, z^{\prime}\right) F\left(B_{\left[0, T_{\partial D_{1}}\right]}\right), T_{\partial D}>T_{\partial D_{1}}\right],
\end{aligned}
$$


which readily gives (i).

(ii) First we suppose that $z$ and $z^{\prime}$ are distinct points of $D_{1}$. By (2.11),

$$
\begin{aligned}
\mathbb{P}_{D}^{z, z^{\prime}}\left(T_{z^{\prime}}>T_{\partial D_{1}}\right) & =\frac{\mathbb{E}_{D}^{z}\left[H_{D}\left(B_{T_{\partial D_{1}}}, z^{\prime}\right), T_{\partial D}>T_{\partial D_{1}}\right]}{H_{D}\left(z, z^{\prime}\right)} \\
& =\frac{\mathbb{E}^{z}\left[G_{D}\left(B_{T_{\partial D_{1}}}, z^{\prime}\right)\right]}{G_{D}\left(z, z^{\prime}\right)} .
\end{aligned}
$$

Note that the function $g$ defined by $g(y):=G_{D}\left(y, z^{\prime}\right)-G_{D_{1}}\left(y, z^{\prime}\right), y \in D_{1} \backslash\left\{z^{\prime}\right\}$, is harmonic and bounded. Then $g$ can be continuously extended to $\left\{z^{\prime}\right\}$ so that $g$ is harmonic on $D_{1}{ }^{6}$ By the optional stopping theorem for the bounded martingale $g\left(B_{t}\right)$, we get that

$$
\mathbb{E}^{z}\left[G_{D}\left(B_{T_{\partial D_{1}}}, z^{\prime}\right)\right]=G_{D}\left(z, z^{\prime}\right)-G_{D_{1}}\left(z, z^{\prime}\right),
$$

proving (ii) in this case. Making $z$ go to $\partial D \cap \partial D_{1}$, using (2.2) and (2.8) yields the equality for $z^{\prime} \in D_{1}$ and $z \in \mathcal{J}\left(D, D_{1}\right)$. Using the time-reversal (2.9) yields the equality for $z \in D_{1}$ and $z^{\prime} \in \mathcal{J}\left(D, D_{1}\right)$. Making $z$ go to $\partial D \cap \partial D_{1}$ and using (2.3) and (2.8) gives the equality for $z, z^{\prime} \in \mathcal{J}\left(D, D_{1}\right)$.

\subsection{Brownian loops}

Let $x \in D$. We consider an infinite measure $\nu_{D}(x, x)$ on the set of Brownian loops in $D$ that start and end at $x$. Let us briefly recall the definition in Lawler ([10], Chapter $5)$. For each $t>0$, the measure $\mu_{D}(x, x ; t)$ of loops in $D$ of length $t$ is such that for any bounded measurable function $\Phi$ on $\mathcal{K}$ :

$$
\mu_{D}(x, x ; t)[\Phi(\mathfrak{e})]=\lim _{\varepsilon \rightarrow 0^{+}} \frac{1}{\pi \varepsilon^{2}} \mathbb{E}^{x}\left[\Phi\left(B_{[0, t]}\right), t<T_{\partial D},\left|B_{t}-x\right|<\varepsilon\right] .
$$

We define the $\sigma$-finite measure $\nu_{D}(x, x)$ by

$$
\nu_{D}(x, x):=\pi \int_{0}^{\infty} \mu_{D}(x, x ; t) \mathrm{d} t .
$$

With the notation $\mu_{D}(x, x)$ of the loop measure in Lawler ([10], Chapter 5), we have $\nu_{D}(x, x)=\pi \mu_{D}(x, x)$.

\footnotetext{
${ }^{6}$ We give here a probabilistic argument on this known continuous extension. Let $z \in D_{1} \backslash\left\{z^{\prime}\right\}$ and let $\varepsilon>0$ be small such that $\mathcal{B}(z, \varepsilon) \subset D_{1}$. By the optional stopping theorem for the bounded martingale $g\left(B_{\left.t \wedge T_{\mathcal{C}\left(z^{\prime}, \varepsilon\right)}\right)}\right)$ at $T_{\partial D_{1}}$ and by letting $\varepsilon \rightarrow 0^{+}$, we get that $g(z)=\mathbb{E}^{z}\left(g\left(B_{T_{\partial D_{1}}}\right)\right)=\int_{\partial D_{1}} H_{D_{1}}(z, y) g(y) \mathrm{d} y$ which can be continuously extended to $z^{\prime}$ and the extension is harmonic on $D_{1}$.
} 
Furthermore, $\nu_{D}(x, x)$ can also be viewed as the excursion measure at $x$ of the Brownian motion conditioned to hit $x$ before hitting $\partial D$. We claim that

$$
\nu_{D}(x, x)=\lim _{z \rightarrow x} \log \left(\frac{1}{|z-x|}\right) \mathbb{P}_{D}^{z, x}
$$

in the sense that for any $r>0$ such that $\mathcal{B}(x, r) \subset D$, for any $\mathbf{A} \in \sigma\left\{\mathfrak{e}_{\mathcal{C}(x, r)+t}, t \geq 0\right\}$,

$$
\nu_{D}(x, x)\left(\mathbf{A} \cap\left\{T_{\mathcal{C}(x, r)}<T_{x}\right\}\right)=\lim _{z \rightarrow x} \log \left(\frac{1}{|z-x|}\right) \mathbb{P}_{D}^{z, x}\left(\mathbf{A} \cap\left\{T_{\mathcal{C}(x, r)}<T_{x}\right\}\right) .
$$

See Appendix A.2 for a justification of (2.14). Equation (2.13) implies that the measure $\nu$ is conformally invariant: if $D$ and $D^{\prime}$ are two nice domains, $\Psi: D \rightarrow D^{\prime}$ a conformal transformation and $x \in D$, the image by $\Psi$ of a loop under $\nu_{D}(x, x)$ is "distributed" as $\nu_{D^{\prime}}(\Psi(x), \Psi(x))$.

We summarize some quantitative results on $\nu_{D}(x, x)$ in the following Lemma:

Lemma 2.3 Let $D_{1} \subset D$ be two nice domains and $x \in D_{1}$.

(i) For any nice point $y \in \partial D_{1}$,

$$
\nu_{D}(x, x)\left(T_{\partial D_{1}}<T_{x}, \mathfrak{e}_{T_{\partial D_{1}}} \in \mathrm{d} y\right)=G_{D}(x, y) H_{D_{1}}(x, y) \mathrm{d} y .
$$

Moreover for any nonnegative measurable function $F$ on $\mathcal{K}$,

$$
\nu_{D}(x, x)\left(F\left(\mathfrak{e}_{\left[0, T_{\partial D_{1}}\right]}\right), T_{\partial D_{1}}<T_{x}\right)=\mathbb{E}^{x}\left[G_{D}\left(x, B_{T_{\partial D_{1}}}\right) F\left(B_{\left[0, T_{\partial D_{1}}\right]}\right)\right] .
$$

(ii) We have

$$
\begin{aligned}
C_{D, D_{1}}(x) & :=\nu_{D}(x, x)\left(T_{\partial D_{1}}<T_{x}\right) \\
& =\int_{\partial D_{1}} G_{D}(x, y) H_{D_{1}}(x, y) \mathrm{d} y \\
& =C_{D_{1}}(x)-C_{D}(x),
\end{aligned}
$$

where $C_{S}(x):=-\int_{\partial S} \log (|x-y|) H_{S}(x, y) \mathrm{d} y$ for any nice domain $S$. Moreover, $C_{D, D_{1}}(x)=\log \left(R_{x, D}\right)-\log \left(R_{x, D_{1}}\right)$ in the case that $D_{1}, D$ are simply connected, where $R_{x, D}$ (resp. $R_{x, D_{1}}$ ) is the conformal radius of $D$ (resp. $D_{1}$ ) seen from $x$.

(iii) For any nonnegative measurable function $F$ on $\mathcal{K}$,

$$
\begin{aligned}
& \nu_{D}(x, x)\left(F\left(\mathfrak{e}_{\left[T_{\partial D_{1}}, T_{x}\right]}\right), T_{\partial D_{1}}<T_{x}\right) \\
= & \int_{\partial D_{1}} \mathbb{E}_{D}^{y, x}\left[F\left(B_{\left[0, T_{x}\right]}\right)\right] G_{D}(x, y) H_{D_{1}}(x, y) \mathrm{d} y .
\end{aligned}
$$

Consequently,

$$
\nu_{D}(x, x)\left(F\left(\mathfrak{e}_{\left[T_{\partial D_{1}}, T_{x}\right]}\right) \mid T_{\partial D_{1}}<T_{x}, \mathfrak{e}_{T_{\partial D_{1}}}=y\right)=\mathbb{E}_{D}^{y, x}\left[F\left(B_{\left[0, T_{x}\right]}\right)\right] .
$$


Proof. (i) By (2.14) and (2.11), for any $r>0$ such that $\mathcal{B}(x, r) \subset D_{1}$,

$$
\begin{aligned}
& \nu_{D}(x, x)\left(F\left(\mathfrak{e}_{\left[T_{\mathcal{C}(x, r)}, T_{\partial D_{1}}\right]}\right), T_{\partial D_{1}}<T_{x}\right) \\
= & \lim _{z \rightarrow x} \frac{\log (1 /|z-x|)}{G_{D}(x, z)} \mathbb{E}^{z}\left[G_{D}\left(x, B_{T_{\partial D_{1}}}\right) F\left(B_{\left[T_{\mathcal{C}(x, r)}, T_{\partial D_{1}}\right]}\right)\right] \\
= & \mathbb{E}^{x}\left[G_{D}\left(x, B_{T_{\partial D_{1}}}\right) F\left(B_{\left[T_{\mathcal{C}(x, r)}, T_{\partial D_{1}}\right]}\right)\right]
\end{aligned}
$$

by (2.1). Under $\mathbb{P}^{x}, B_{T_{\partial D_{1}}}$ is distributed as $H_{D_{1}}(x, y) \mathrm{d} y$, which implies the first equality in (i) by taking $F\left(B_{\left[T_{\mathcal{C}(x, r)}, T_{\partial D_{1}}\right]}\right)$ a function of $B_{T_{\partial D_{1}}}$. By considering $F$ a continuous and bounded function on $\mathcal{K}$, and then letting $r \rightarrow 0^{+}$, we get the second equality in (i).

(ii) The first equality in (2.15) readily follows from (i). Let $g(y):=G_{D}(x, y)-G_{D_{1}}(x, y)$ for $y \in D_{1} \backslash\{x\}\left[x \in D_{1}\right.$ being fixed]. We have already observed that $g$ can be continuously extended to $x$. Then $g$ is harmonic and bounded on $D_{1}$, and by the mean property,

$$
g(x)=\int_{\partial D_{1}} g(y) H_{D_{1}}(x, y) \mathrm{d} y=\int_{\partial D_{1}} G_{D}(x, y) H_{D_{1}}(x, y) \mathrm{d} y .
$$

The same argument shows that, for $S=D$ or $S=D_{1}$,

$$
\int_{\partial S} \log (|x-y|) H_{S}(x, y) d y=\lim _{y \rightarrow x}\left(\log (|x-y|)+G_{S}(x, y)\right),
$$

which implies that $C_{D, D_{1}}(x)=C_{D_{1}}(x)-C_{D}(x)$, as stated in (2.15).

Now we suppose that $D$ is simply connected. It suffices to check that

$$
C_{D}(x)=-\log \left(R_{x, D}\right) .
$$

Let $\Phi_{D}$ be a conformal map from $D$ (resp. $D_{1}$ ) to $\mathcal{B}(0,1)$ which sends $x$ to 0 and satisfies that $\Phi_{D}^{\prime}(x)>0$. By the conformal invariance, $G_{D}(x, y)=\log \frac{1}{\left|\Phi_{D}(y)\right|}$. It follows that

$$
\lim _{y \rightarrow x}\left(\log (|x-y|)+G_{D}(x, y)\right)=\log \frac{1}{\left|\Phi_{D}^{\prime}(x)\right|}=\log R_{x, D}
$$

by definition of the conformal radius.

(iii) It comes from a straightforward application of the strong Markov property together with the first equality in (i).

\section{A change of measures}

Let $\alpha \geq 0$ be fixed. Let $D$ be a nice domain. We consider $D_{1} \subset D$ a nice domain containing $x$. We define for any distinct nice points $y, z \in \bar{D}_{1}$, different from $x$,

$$
\xi_{D_{1}}(x, y, z):=\frac{2 \pi H_{D_{1}}(x, y) H_{D_{1}}(x, z)}{H_{D_{1}}(y, z)} .
$$


[Taking $D_{1}=D$, we get the definition of $\xi_{D}(x, y, z)$ for any $x \in D$ and distinct nice points $y, z \in \bar{D}$, different from $x]$.

Fix two nice points $z \neq z^{\prime}$ of $\bar{D}$. Let $x \in D$ distinct of $z$ and $z^{\prime}$. Let us take a close look at the probability measure $\mathbb{Q}_{x, D}^{z, z^{\prime}, \alpha}$ defined in the Introduction. Under $\mathbb{Q}_{x, D}^{z, z^{\prime}, \alpha}$, the Brownian motion $B$ starts from $z$ and is conditioned to hit $x$. After $T_{x}$, the trajectory is a concatenation of Brownian loops generated by a Poisson point process $\left(\mathfrak{e}_{s}\right)_{s \geq 0}$ with intensity $1_{[0, \alpha]} \mathrm{d} t \times \nu_{D}(x, x)$. The last part of trajectory is a Brownian motion in $D$ starting from $x$ and conditioned to hit $z^{\prime}$.

The purpose of this section is to study the absolute continuity of $\mathbb{Q}_{x, D}^{z, z^{\prime}, \alpha}$ with respect to $\mathbb{P}_{D}^{z, z^{\prime}}$, both restricted to the sigma-algebra generated by the excursions outside a domain $D_{1}$ containing $x$.

As a first step, we look at events of crossings. We introduce some notation. Let $\mathcal{K}$ denote as before the set of all parametrized continuous planar curves $\gamma$ defined on a finite time-interval $\left[0, t_{\gamma}\right]$ with $t_{\gamma} \in(0, \infty)$.

Notation 3.1 Let $D_{2} \subset D_{1} \subset D$ be nice domains with $d\left(D_{2}, \partial D_{1}\right)>0$. Let $\gamma \in \mathcal{K}$.

(i) Define $\mathbf{s}_{0}:=0$ and for all $i \geq 0$ (with inf $\varnothing:=\infty$ ),

$$
\begin{aligned}
\mathbf{t}_{i+1} & :=\inf \left\{t>\mathbf{s}_{i}: \gamma(t) \in \partial D_{2}\right\}, \\
\mathbf{s}_{i+1} & :=\inf \left\{t>\mathbf{t}_{i+1}: \gamma(t) \in \partial D_{1} \cup\left\{\gamma\left(t_{\gamma}\right)\right\}\right\} .
\end{aligned}
$$

(ii) Let $x \in D_{2}$. For any integer $\ell \geq 1$, denote, if it exists, by $\mathbf{i}_{\ell}$ the $\ell$-th smallest index $i \geq 1$ such that $\gamma$ hits $x$ during the time interval $\left[\mathbf{t}_{i}, \mathbf{s}_{i}\right]$ and set $\mathbf{U}_{\ell}:=\mathbf{t}_{\mathbf{i}_{\ell}}, \mathbf{V}_{\ell}:=\mathbf{s}_{\mathbf{i}_{\ell}}$.

Let $D_{2} \subset D_{1} \subset D$ be nice domains with $d\left(D_{2}, \partial D_{1}\right)>0$. Let $x \in D_{2}$ and $z \neq z^{\prime}$ be nice points of $\bar{D}$ different from $x$, neither in $\partial D_{1} \backslash \mathcal{J}\left(D, D_{1}\right)$ nor in $\overline{D_{2}} 7$.

Let

$$
\mathbf{L}:=1+\#\left\{\text { loops from } x \text { which hit } \partial D_{1}\right\}
$$

By Lemma 2.3,

$$
\mathbb{Q}_{x, D}^{z, z^{\prime}, \alpha}(\mathbf{L}=L)=\mathrm{e}^{-\alpha C_{D, D_{1}}(x)} \frac{\left(\alpha C_{D, D_{1}}(x)\right)^{L-1}}{(L-1) !} .
$$

Notice that $\mathbf{i}_{\ell}$ is well-defined for any $1 \leq \ell \leq \mathbf{L}$. The next proposition gives the law of the points $\left(B_{\mathbf{U}_{\ell}}, B_{\mathbf{V}_{\ell}}\right)_{\ell \leq \mathbf{L}}$ under $\mathbb{Q}_{x, D}^{z, z^{\prime}, \alpha}$. Notice that we may have $B_{\mathbf{V}_{\mathbf{L}}}=z^{\prime}$ in the case where $z^{\prime} \in \overline{D_{1}}$ and the excursion from $x$ to $z^{\prime}$ does not hit $\partial D_{1}$ (except, of course, possibly at the ending point).

\footnotetext{
${ }^{7}$ In other words, $z, z^{\prime} \in \mathcal{J}\left(D, D_{1}\right) \cup\left(\bar{D} \backslash\left(\{x\} \cup \partial D_{1} \cup \overline{D_{2}}\right)\right)$. Note that $z \notin \partial D_{1} \backslash \mathcal{J}\left(D, D_{1}\right)$ means that $z$ does not belong to $\partial D_{1}$ unless $z \in \mathcal{J}\left(D, D_{1}\right)$.
} 


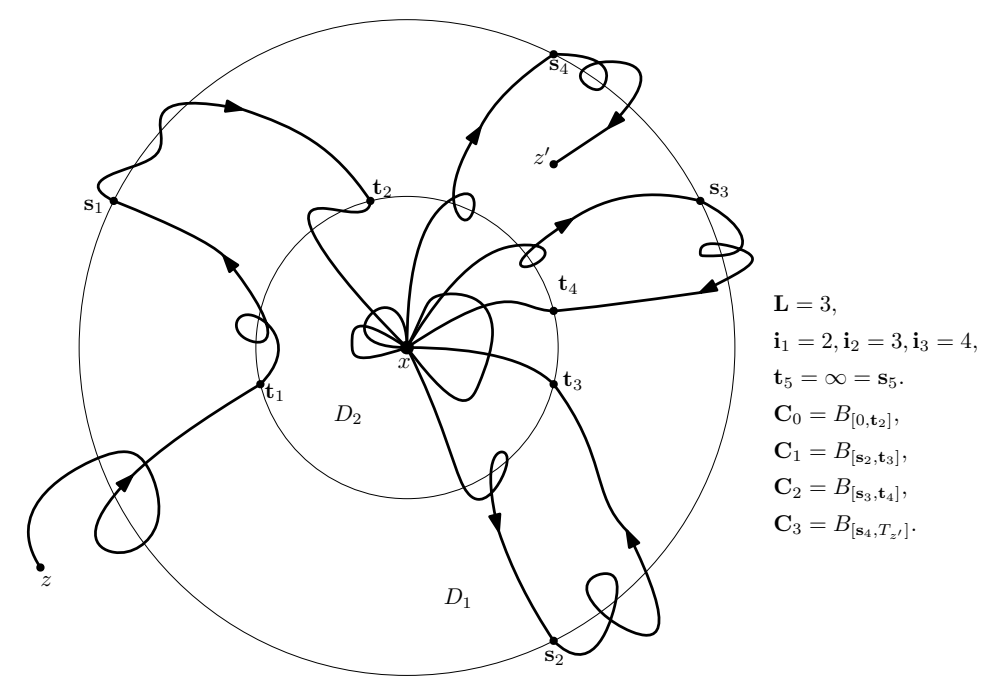

Proposition 3.2 Let $D_{2} \subset D_{1} \subset D$ nice domains with $d\left(D_{2}, \partial D_{1}\right)>0$. Let $x \in D_{2}$. Let $z \neq z^{\prime}$ be nice points of $\bar{D}$ different from $x$, neither in $\partial D_{1} \backslash \mathcal{J}\left(D, D_{1}\right)$ nor in $\overline{D_{2}} .8$ Let $L \geq 1$ be an integer. Let $y_{1}, \ldots, y_{L}$ be nice points of $\partial D_{2}$. Let $y_{1}^{\prime}, \ldots, y_{L}^{\prime}$ be nice points of $\partial D_{1} \backslash \partial D$. We can take as well $y_{L}^{\prime}=z^{\prime}$ in the case $z^{\prime} \in D_{1} \cup \mathcal{J}\left(D, D_{1}\right)$. Let $1 \leq i_{1}<\ldots<i_{L}$ be integers. Then

$$
\begin{aligned}
& \mathbb{Q}_{x, D}^{z, z^{\prime}, \alpha}\left(\mathbf{i}_{j}=i_{j}, B_{\mathbf{U}_{j}} \in \mathrm{d} y_{j}, B_{\mathbf{V}_{j}} \in \mathrm{d} y_{j}^{\prime}, 1 \leq j \leq L \mid \mathbf{L}=L\right) \\
= & \frac{C_{D, D_{1}}(x)^{-(L-1)}}{\xi_{D}\left(x, z, z^{\prime}\right)} \times \\
& \prod_{j=1}^{L} \xi_{D_{1}}\left(x, y_{j}, y_{j}^{\prime}\right) \mathbb{P}_{D}^{z, z^{\prime}}\left(B_{\mathbf{t}_{i_{j}}} \in \mathrm{d} y_{j}, B_{\mathbf{s}_{i_{j}}} \in \mathrm{d} y_{j}^{\prime}, 1 \leq j \leq L, \mathbf{t}_{i_{L}}<T_{z^{\prime}}\right) .
\end{aligned}
$$

In this equation, we mean, in the case $y_{L}^{\prime}=z^{\prime}, B_{\mathbf{V}_{L}}=z^{\prime}$ and $B_{\mathbf{s}_{i_{L}}}=z^{\prime}$ when writing $B_{\mathbf{V}_{L}} \in \mathrm{d} y_{L}^{\prime}$ and $B_{\mathbf{s}_{L}} \in \mathrm{d} y_{L}^{\prime}$.

Taking $L=1$ and integrating over $y_{j}$ and $y_{j}^{\prime}$ yields the following equation which will be used in (3.12):

$$
\xi_{D}\left(x, z, z^{\prime}\right)=\mathbb{E}_{D}^{z, z^{\prime}}\left[\sum_{i \geq 1} \xi_{D_{1}}\left(x, B_{\mathbf{t}_{i}}, B_{\mathbf{s}_{i}}\right) 1_{\left\{\mathbf{t}_{i}<T_{z^{\prime}}\right\}}\right] .
$$

Proof of Proposition 3.2. To deal with the case $z \in \partial D$, we extend the $h$-transform representation (2.7) to $z \in \partial D$. To this end, we abusively write, when $z \in \partial D, \mathbb{P}_{D}^{z}$ for

\footnotetext{
${ }^{8}$ The assumption $z, z^{\prime} \notin \partial D_{1} \backslash \mathcal{J}\left(D, D_{1}\right)$, which may look somehow uncomfortable, will be automatically satisfied in the applications in the forthcoming sections. The same remark applies to other propositions and corollaries in this section.
} 
$\nu_{D}(z)$ defined in (A.2) (which is not a finite measure). With this notation, (2.7) still holds for nice point $z$ of $\partial D$ : this comes from (2.8), (2.7) (replacing $z$ there by $z+\varepsilon \mathbf{n}_{z}$ ), and (A.2).

Denote by $Q$ the probability expression $\mathbb{Q}_{x, D}^{z, z^{\prime}, \alpha}(\ldots)$ in the proposition. For simplicity, we suppose first that $y_{L}^{\prime} \neq z^{\prime}$. We notice that for each $1 \leq \ell \leq L-1$, the trajectory between $\mathbf{V}_{\ell}$ and the next hitting time of $x$ is, conditionally on $B_{\mathbf{V}_{\ell}}=y^{\prime}$, distributed as $\mathbb{P}_{D}^{y^{\prime}, x}$. Moreover, conditionally on $\mathbf{L}=L, B_{\mathbf{V}_{\ell}}$ is distributed as $\mathfrak{e}_{T_{\partial D_{1}}}$ under $\nu_{D}(x, x)(\cdot \mid$ $T_{\partial D_{1}}<T_{x}$ ) when $\ell<L$, and as $B_{T_{\partial D_{1} \cup\left\{z^{\prime}\right\}}}$ under $\mathbb{P}_{D}^{x, z^{\prime}}$ when $\ell=L$. Using the strong Markov property at the hitting times of $x$ and stopping times $\mathbf{V}_{1}, \ldots, \mathbf{V}_{L-1}$, the probability expression $Q$ is

$$
\begin{aligned}
& \prod_{j=1}^{L-1} \mathbb{P}_{D}^{y_{j-1}^{\prime}, x}\left(\mathbf{i}_{1}=i_{j}-i_{j-1}, B_{\mathbf{U}_{1}} \in \mathrm{d} y_{j}\right) \nu_{D}(x, x)\left(\mathfrak{e}_{T_{\partial D_{1}}} \in \mathrm{d} y_{j}^{\prime} \mid B_{T_{\partial D_{1}}}<T_{x}\right) \\
& \times \mathbb{P}_{D}^{y_{L-1}^{\prime}, x}\left(\mathbf{i}_{1}=i_{L}-i_{L-1}, B_{\mathbf{U}_{1}} \in \mathrm{d} y_{L}\right) \mathbb{P}_{D}^{x, z^{\prime}}\left(B_{T_{\partial D_{1}} \cup\left\{z^{\prime}\right\}} \in \mathrm{d} y_{L}^{\prime}\right)
\end{aligned}
$$

where we set $y_{0}^{\prime}=z$ and $i_{0}=0$. We can write it as

$$
Q=\Pi_{1} \Pi_{2}
$$

where

$$
\begin{aligned}
\Pi_{1} & :=\prod_{j=1}^{L} \mathbb{P}_{D}^{y_{j-1}^{\prime}, x}\left(\mathbf{i}_{1}=i_{j}-i_{j-1}, B_{\mathbf{U}_{1}} \in \mathrm{d} y_{j}\right), \\
\Pi_{2} & :=\prod_{j=1}^{L-1} \nu_{D}(x, x)\left(\mathfrak{e}_{T_{\partial D_{1}}} \in \mathrm{d} y_{j}^{\prime} \mid T_{\partial D_{1}}<T_{x}\right) \mathbb{P}_{D}^{x, z^{\prime}}\left(B_{T_{\partial D_{1}} \cup\left\{z^{\prime}\right\}} \in \mathrm{d} y_{L}^{\prime}\right) .
\end{aligned}
$$

By the strong Markov property at the stopping time $\mathbf{t}_{i}$, we have for any $y^{\prime} \in \partial D_{1} \cup\{z\}$, $y \in \partial D_{2}, \mathbb{P}_{D}^{y^{\prime}, x}\left(\mathbf{i}_{1}=i, B_{\mathbf{U}_{1}} \in \mathrm{d} y\right)=\mathbb{P}_{D}^{y^{\prime}, x}\left(B_{\mathbf{t}_{i}} \in \mathrm{d} y, \mathbf{t}_{i}<T_{x}\right) \mathbb{P}_{D}^{y, x}\left(T_{x}<T_{\partial D_{1}}\right)$ and by Lemma 2.2 (ii) (with $z=y, z^{\prime}=x$ there), $\mathbb{P}_{D}^{y, x}\left(T_{x}<T_{\partial D_{1}}\right)=\frac{H_{D_{1}}(y, x)}{H_{D}(y, x)}$. We get that

$$
\mathbb{P}_{D}^{y^{\prime}, x}\left(\mathbf{i}_{1}=i, B_{\mathbf{U}_{1}} \in \mathrm{d} y\right)=\mathbb{P}_{D}^{y^{\prime}, x}\left(B_{\mathbf{t}_{i}} \in \mathrm{d} y, \mathbf{t}_{i}<T_{x}\right) \frac{H_{D_{1}}(y, x)}{H_{D}(y, x)}
$$

which is, using the $h$-transform (2.7),

$$
\mathbb{P}_{D}^{y^{\prime}}\left(B_{\mathbf{t}_{i}} \in \mathrm{d} y, \mathbf{t}_{i}<T_{\partial D}\right) \frac{H_{D_{1}}(y, x)}{H_{D}\left(y^{\prime}, x\right)} .
$$

Hence,

$$
\Pi_{1}=\prod_{j=1}^{L} \mathbb{P}_{D}^{y_{j-1}^{\prime}}\left(B_{\mathbf{t}_{i_{j}-i_{j-1}}} \in \mathrm{d} y_{j}, \mathbf{t}_{i_{j}-i_{j-1}}<T_{\partial D}\right) \frac{H_{D_{1}}\left(y_{j}, x\right)}{H_{D}\left(y_{j-1}^{\prime}, x\right)}
$$


On the other hand, the law of $\mathfrak{e}_{T_{\partial D_{1}}}$ under $\nu_{D}(x, x)\left(\cdot \mid T_{\partial D_{1}}<T_{x}\right)$ has, by Lemma 2.3, density on $\partial D_{1}$

$$
\frac{1}{C_{D, D_{1}}(x)} G_{D}\left(x, y^{\prime}\right) H_{D_{1}}\left(x, y^{\prime}\right) \mathrm{d} y^{\prime},
$$

whereas, by Lemma 2.2 (i) (with $z=x, z^{\prime}=z^{\prime}$ there),

$$
\mathbb{P}_{D}^{x, z^{\prime}}\left(B_{T_{\partial D_{1}}} \in \mathrm{d} y^{\prime}, T_{\partial D_{1}}<T_{z^{\prime}}\right)=\frac{H_{D_{1}}\left(x, y^{\prime}\right) H_{D}\left(y^{\prime}, z^{\prime}\right)}{H_{D}\left(x, z^{\prime}\right)} \mathrm{d} y^{\prime} .
$$

Recalling $y_{L}^{\prime} \neq z^{\prime}$, we obtain that

$$
\Pi_{2}=C_{D, D_{1}}(x)^{-(L-1)} \frac{H_{D_{1}}\left(x, y_{L}^{\prime}\right) H_{D}\left(y_{L}^{\prime}, z^{\prime}\right)}{H_{D}\left(x, z^{\prime}\right)} \mathrm{d} y_{L}^{\prime} \prod_{j=1}^{L-1} G_{D}\left(x, y_{j}^{\prime}\right) H_{D_{1}}\left(x, y_{j}^{\prime}\right) \mathrm{d} y_{j}^{\prime} .
$$

Recall that $G_{D}\left(y, y^{\prime}\right)=2 \pi H_{D}\left(y, y^{\prime}\right)$ when $y, y^{\prime} \in D$. By (3.3), (3.4) and (3.6), we get that

$$
\begin{aligned}
Q= & (2 \pi)^{L-1} C_{D, D_{1}}(x)^{-(L-1)} \frac{H_{D}\left(y_{L}^{\prime}, z^{\prime}\right)}{H_{D}(z, x) H_{D}\left(x, z^{\prime}\right)} \times \\
& \prod_{j=1}^{L} H_{D_{1}}\left(x, y_{j}\right) H_{D_{1}}\left(x, y_{j}^{\prime}\right) \mathrm{d} y_{j}^{\prime} \prod_{j=1}^{L} \mathbb{P}_{D}^{y_{j-1}^{\prime}\left(B_{\mathbf{t}_{i_{j}-i_{j-1}}} \in \mathrm{d} y_{j}, \mathbf{t}_{i_{j}-i_{j-1}}<T_{\partial D}\right) .}
\end{aligned}
$$

In view of the definition of $\xi_{D_{1}}$ in $(3.1)$, we have

$$
\begin{aligned}
& (2 \pi)^{L-1} \frac{H_{D}\left(y_{L}^{\prime}, z^{\prime}\right)}{H_{D}(z, x) H_{D}\left(x, z^{\prime}\right)} \prod_{j=1}^{L} H_{D_{1}}\left(x, y_{j}\right) H_{D_{1}}\left(x, y_{j}^{\prime}\right) \\
= & \frac{H_{D}\left(y_{L}^{\prime}, z^{\prime}\right)}{H_{D}\left(z, z^{\prime}\right) \xi_{D}\left(x, z, z^{\prime}\right)} \prod_{j=1}^{L} \xi_{D_{1}}\left(x, y_{j}, y_{j}^{\prime}\right) \prod_{j=1}^{L} H_{D_{1}}\left(y_{j}, y_{j}^{\prime}\right) .
\end{aligned}
$$

Comparing with the statement of the Proposition, we see that it remains to prove that

$$
\begin{aligned}
& H_{D}\left(y_{L}^{\prime}, z^{\prime}\right) \prod_{j=1}^{L} \mathbb{P}_{D}^{y_{j-1}^{\prime}}\left(B_{\mathbf{t}_{i_{j}-i_{j-1}}} \in \mathrm{d} y_{j}, \mathbf{t}_{i_{j}-i_{j-1}}<T_{\partial D}\right) H_{D_{1}}\left(y_{j}, y_{j}^{\prime}\right) \mathrm{d} y_{j}^{\prime} \\
= & H_{D}\left(z, z^{\prime}\right) \mathbb{P}_{D}^{z, z^{\prime}}\left(B_{\mathbf{t}_{i_{j}}} \in \mathrm{d} y_{j}, B_{\mathbf{s}_{i_{j}}} \in \mathrm{d} y_{j}^{\prime}, 1 \leq j \leq L, \mathbf{t}_{i_{L}}<T_{z^{\prime}}\right) .
\end{aligned}
$$

Consider the right-hand side. By the $h$-transform (2.7) (we recall that $y_{L}^{\prime} \neq z^{\prime}$ hence $\left.\mathbf{s}_{i_{L}}<T_{z^{\prime}}\right)$, it is equal to

$$
\mathbb{P}_{D}^{z}\left(B_{\mathbf{t}_{i_{j}}} \in \mathrm{d} y_{j}, B_{\mathbf{s}_{i_{j}}} \in \mathrm{d} y_{j}^{\prime}, 1 \leq j \leq L, \mathbf{s}_{i_{L}}<T_{\partial D}\right) H_{D}\left(y_{L}^{\prime}, z^{\prime}\right)
$$


Recall that $H_{D_{1}}\left(y, y^{\prime}\right)$ for $y \in D_{1}$ and $y^{\prime} \in \partial D_{1}$ is the density at $y^{\prime}$ of the harmonic measure of the Brownian motion starting at $y$. It follows that, using the strong Markov property at the hitting times $\mathbf{s}_{i_{j-1}}, j \geq 2$, and $\mathbf{t}_{i_{j}}, j \geq 1$,

$$
\begin{aligned}
& \mathbb{P}_{D}^{z}\left(B_{\mathbf{t}_{i_{j}}} \in \mathrm{d} y_{j}, B_{\mathbf{s}_{i_{j}}} \in \mathrm{d} y_{j}^{\prime}, 1 \leq j \leq L, \mathbf{s}_{i_{L}}<T_{\partial D}\right) \\
= & \prod_{j=1}^{L} \mathbb{P}_{D}^{y_{j-1}^{\prime}}\left(B_{\mathbf{t}_{i_{j}-i_{j-1}}} \in \mathrm{d} y_{j}, \mathbf{t}_{i_{j}-i_{j-1}}<T_{\partial D}\right) H_{D_{1}}\left(y_{j}, y_{j}^{\prime}\right) \mathrm{d} y_{j}^{\prime}
\end{aligned}
$$

which completes the proof in the case $y_{L}^{\prime} \neq z^{\prime}$. In the remaining case $y_{L}^{\prime}=z^{\prime}$, the same proof applies by replacing (3.5) by

$$
\mathbb{P}_{D}^{x, z^{\prime}}\left(T_{z^{\prime}} \leq T_{\partial D_{1}}\right)=\frac{H_{D_{1}}\left(x, z^{\prime}\right)}{H_{D}\left(x, z^{\prime}\right)}
$$

which holds by Lemma 2.2 (ii) (with $z=x$ and $z^{\prime}=z^{\prime}$ there), then replacing (3.7) by

$$
\begin{aligned}
& \prod_{j=1}^{L} \mathbb{P}_{D}^{y_{j-1}^{\prime}}\left(B_{\mathbf{t}_{i_{j}-i_{j-1}}} \in \mathrm{d} y_{j}, \mathbf{t}_{i_{j}-i_{j-1}}<T_{\partial D}\right) H_{D_{1}}\left(y_{j}, y_{j}^{\prime}\right) \prod_{j=1}^{L-1} \mathrm{~d} y_{j}^{\prime} \\
= & H_{D}\left(z, z^{\prime}\right) \mathbb{P}_{D}^{z, z^{\prime}}\left(B_{\mathbf{t}_{i_{j}}} \in \mathrm{d} y_{j}, B_{\mathbf{s}_{i_{j}}} \in \mathrm{d} y_{j}^{\prime}, 1 \leq j \leq L, \mathbf{t}_{i_{L}}<T_{z^{\prime}}\right)
\end{aligned}
$$

where we write $B_{\mathbf{s}_{i_{L}}} \in \mathrm{d} y_{L}^{\prime}$ for $B_{\mathbf{s}_{i_{L}}}=z^{\prime}$.

As in the case $y_{L}^{\prime} \neq z^{\prime}$, it remains to check (3.8). By the strong Markov property at time $\mathbf{t}_{i_{L}}$ and Lemma 2.2 (ii) (with $z=y_{L}$ and $z^{\prime}=z^{\prime}$ there), the right-hand side in (3.8) is

$$
\begin{aligned}
& H_{D}\left(z, z^{\prime}\right) \times \\
& \mathbb{P}_{D}^{z, z^{\prime}}\left(B_{\mathbf{t}_{i_{j}}} \in \mathrm{d} y_{j}, B_{\mathbf{s}_{i_{j}}} \in \mathrm{d} y_{j}^{\prime}, 1 \leq j \leq L-1, B_{\mathbf{t}_{i_{L}}} \in \mathrm{d} y_{L}, \mathbf{t}_{i_{L}}<T_{z^{\prime}}\right) \times \\
& \frac{H_{D_{1}}\left(y_{L}, z^{\prime}\right)}{H_{D}\left(y_{L}, z\right)}
\end{aligned}
$$

which, in view of the $h$-transform (2.7), is

$$
\begin{aligned}
& \mathbb{P}_{D}^{z}\left(B_{\mathbf{t}_{i_{j}}} \in \mathrm{d} y_{j}, B_{\mathbf{s}_{i_{j}}} \in \mathrm{d} y_{j}^{\prime}, 1 \leq j \leq L-1, B_{\mathbf{t}_{i_{L}}} \in \mathrm{d} y_{L}, \mathbf{t}_{i_{L}}<T_{\partial D}\right) \times \\
& H_{D_{1}}\left(y_{L}, z^{\prime}\right) .
\end{aligned}
$$

We use, as before, the strong Markov property at times $\mathbf{s}_{i_{j-1}}, j \geq 2$ and $\mathbf{t}_{i_{j}}, j \geq 1$ to get (3.8). This completes the proof of Proposition 3.2.

Our next aim is to compute the Radon-Nikodym derivative of $\mathbb{Q}_{x, D}^{z, z^{\prime}, \alpha}$ with respect to $\mathbb{P}_{D}^{z, z^{\prime}}$ when both measures are restricted to an appropriate sigma-algebra denoted by $\mathscr{F}_{D_{1}}^{+}$ 
(defined in Notation 3.4 below). We start by computing the conditional law of $\mathbb{Q}_{x, D}^{z, z^{\prime}, \alpha}$ given $\mathbf{L}$.

Take the notation of Proposition 3.2. Fix an arbitrary $L \geq 1$ as well as arbitrary integers $1 \leq i_{1}<i_{2}<\cdots<i_{L}$. On the event $\left\{\mathbf{t}_{i_{L}}<T_{z^{\prime}}\right\}$, let $\mathbf{C}_{j}$ be the trajectory from $\mathbf{s}_{i_{j}}$ to $\mathbf{t}_{i_{j+1}}$ for $1 \leq j \leq L-1, \mathbf{C}_{0}$ the trajectory from (time) 0 to $\mathbf{t}_{i_{1}}$, and $\mathbf{C}_{L}$ the trajectory from $\mathbf{s}_{i_{L}}$ to $T_{z^{\prime}}$. [In case $\mathbf{t}_{i_{L}} \geq T_{z^{\prime}}, \mathbf{C}_{j}$ will play no role, and can be defined as any trajectories.]

Observe that, under $\mathbb{Q}_{x, D}^{z, z^{\prime}, \alpha}$, conditionally on $\mathbf{L}=L$ and on $\mathbf{i}_{j}=i_{j}, B_{\mathbf{U}_{j}} \in \mathrm{d} y_{j}$, $B_{\mathbf{V}_{j}} \in \mathrm{d} y_{j}^{\prime}, 1 \leq j \leq L$ (in particular, $\mathbf{t}_{i_{L}}<T_{z^{\prime}}$ ), the trajectories $\left(\mathbf{C}_{j}, 0 \leq j \leq L\right.$ ) are independent; for $0 \leq j \leq L-1, \mathbf{C}_{j}$ is distributed as a Brownian excursion in $D$ from $y_{j}^{\prime}$ to $y_{j+1}$ conditioned on $\mathbf{t}_{i_{j+1}-i_{j}}=T_{y_{j+1}}$ (with $i_{0}:=0$ and $y_{0}^{\prime}:=z$ ); $\mathbf{C}_{L}$ is a Brownian excursion in $D$ from $y_{L}^{\prime}$ to $z^{\prime}$. [Beware of the degenerate situation that $\mathbf{C}_{L}=\left\{z^{\prime}\right\}$ if $y_{L}^{\prime}=z^{\prime}$.] In other words, $\left(\mathbf{C}_{j}, 0 \leq j \leq L\right)$ has the same distribution under $\mathbb{Q}_{x, D}^{z, z^{\prime}, \alpha}(\cdot \mid$ $\left.\mathbf{L}=L, \mathbf{i}_{j}=i_{j}, B_{\mathbf{U}_{j}} \in \mathrm{d} y_{j}, B_{\mathbf{V}_{j}} \in \mathrm{d} y_{j}^{\prime}, 1 \leq j \leq L\right)$ and under $\mathbb{P}_{D}^{z, z^{\prime}}\left(\cdot \mid B_{\mathbf{t}_{i_{j}}} \in \mathrm{d} y_{j}, B_{\mathbf{s}_{i_{j}}} \in\right.$ $\left.\mathrm{d} y_{j}^{\prime}, 1 \leq j \leq L, \mathbf{t}_{i_{L}}<T_{z^{\prime}}\right)$. It implies that for any measurable set $A \in \sigma\left(\mathbf{C}_{j}, 0 \leq j \leq L\right)$,

$$
\begin{aligned}
& \mathbb{Q}_{x, D}^{z, z^{\prime}, \alpha}\left(A \mid \mathbf{L}=L, \mathbf{i}_{j}=i_{j}, B_{\mathbf{U}_{j}} \in \mathrm{d} y_{j}, B_{\mathbf{V}_{j}} \in \mathrm{d} y_{j}^{\prime}, 1 \leq j \leq L\right) \\
= & \mathbb{P}_{D}^{z, z^{\prime}}\left(A \mid B_{\mathbf{t}_{i_{j}}} \in \mathrm{d} y_{j}, B_{\mathbf{s}_{i_{j}}} \in \mathrm{d} y_{j}^{\prime}, 1 \leq j \leq L, \mathbf{t}_{i_{L}}<T_{z^{\prime}}\right) .
\end{aligned}
$$

We introduce the following notation. Let $\mathcal{K}$ denote as before the set of all parametrized continuous planar curves $\gamma$ defined on a finite time-interval $\left[0, t_{\gamma}\right]$ with $t_{\gamma} \in(0, \infty)$.

Notation 3.3 Let $D_{1} \subset D$ be nice domains. Let $\gamma \in \mathcal{K}$.

(i) Let $\mathcal{E}_{D_{1}}$ be the set of excursions away from $D_{1}^{c} \cup\{\gamma(0)\} \cup\left\{\gamma\left(t_{\gamma}\right)\right\}$ in the sense of (2.6); an element of $\mathcal{E}_{D_{1}}$ is called an excursion inside $D_{1}$.

(ii) For $\mathfrak{e} \in \mathcal{E}_{D_{1}}$, call $\mathfrak{e}_{g}$ and $\mathfrak{e}_{d}$ its starting and ending points.

(iii) An excursion away from $\overline{D_{1}} \cup\{\gamma(0)\} \cup\left\{\gamma\left(t_{\gamma}\right)\right\}$ is called an excursion outside $D_{1}$.

Notation 3.4 Let $D_{1} \subset D$ be nice domains. Let $\mathscr{F}_{D_{1}}^{+}$be the sigma-algebra generated by the excursions of Brownian motion outside $D_{1}$ together with the order of their appearances. $^{9}$

\footnotetext{
${ }^{9}$ For any $\gamma \in \mathcal{K}$, we can associate a function $\phi(\gamma)=\left(\left(\mathfrak{e}_{g}, \mathfrak{e}_{d}\right)_{D_{1}}, \mathcal{R}\right)$ where $\left(\mathfrak{e}_{g}, \mathfrak{e}_{d}\right)_{D_{1}}$ are starting and ending points of excursions in $D_{1}$ of $\gamma$ and $\mathcal{R}$ is the order relation defined as $\mathcal{R}\left(\mathfrak{e}_{g}, \mathfrak{e}_{g}^{\prime}\right)=0$ if $\mathfrak{e}_{g}$ is visited before $\mathfrak{e}_{g}^{\prime}$, and 1 otherwise, for any $\mathfrak{e}_{g}, \mathfrak{e}_{g}^{\prime}$ starting points of excursions in $\mathcal{E}_{D_{1}}$. Then the sigma-algebra generated by $\left(\mathfrak{e}_{g}, \mathfrak{e}_{d}\right)$ for $\mathfrak{e} \in \mathcal{E}_{D_{1}}$ with the order of their appearances, is the sigma-algebra generated by $\phi$.
} 
Observe that for any $A \in \mathscr{F}_{D_{1}}^{+}, A \cap\left\{\mathbf{t}_{i_{L}}<T_{z^{\prime}}\right\} \in \sigma\left(\mathbf{C}_{j}, 0 \leq j \leq L\right)$. Hence, by Proposition 3.2 , we have for any $A \in \mathscr{F}_{D_{1}}^{+}$,

$$
\begin{aligned}
& \mathbb{Q}_{x, D}^{z, z^{\prime}, \alpha}\left(A, \mathbf{i}_{j}=i_{j}, B_{\mathbf{U}_{j}} \in \mathrm{d} y_{j}, B_{\mathbf{V}_{j}} \in \mathrm{d} y_{j}^{\prime}, 1 \leq j \leq L \mid \mathbf{L}=L\right) \\
= & \frac{C_{D, D_{1}}(x)^{-(L-1)}}{\xi_{D}\left(x, z, z^{\prime}\right)} \prod_{j=1}^{L} \xi_{D_{1}}\left(x, y_{j}, y_{j}^{\prime}\right) \times \\
& \mathbb{P}_{D}^{z, z^{\prime}}\left(A, B_{\mathbf{t}_{i_{j}}} \in \mathrm{d} y_{j}, B_{\mathbf{s}_{i_{j}}} \in \mathrm{d} y_{j}^{\prime}, 1 \leq j \leq L, \mathbf{t}_{i_{L}}<T_{z^{\prime}}\right),
\end{aligned}
$$

which gives that

$$
\begin{aligned}
& \mathbb{Q}_{x, D}^{z, z^{\prime}, \alpha}(A \mid \mathbf{L}=L) \\
= & \frac{C_{D, D_{1}}(x)^{-(L-1)}}{\xi_{D}\left(x, z, z^{\prime}\right)} \mathbb{E}_{D}^{z, z^{\prime}}\left[1_{A} \sum_{1 \leq i_{1}<\ldots<i_{L}} \prod_{j=1}^{L} \xi_{D_{1}}\left(x, B_{\mathbf{t}_{i_{j}}}, B_{\mathbf{s}_{i_{j}}}\right) 1_{\left\{\mathbf{t}_{i_{L}}<T_{z^{\prime}}\right\}}\right] .
\end{aligned}
$$

Contrarily to the left-hand side in (3.11), the right-hand side depends on $D_{2}$ via the stopping times. To get rid of the dependence on $D_{2}$, we want to compute

$$
\mathbb{E}_{D}^{z, z^{\prime}}\left[\sum_{1 \leq i_{1}<\ldots<i_{L}} \prod_{j=1}^{L} \xi_{D_{1}}\left(x, B_{\mathbf{t}_{i_{j}}}, B_{\mathbf{s}_{i_{j}}}\right) 1_{\left\{\mathbf{t}_{i_{L}}<T_{z^{\prime}}\right\}} \mid \mathscr{F}_{D_{1}}^{+}\right] .
$$

Observe that any $\left(\mathfrak{e}_{g}, \mathfrak{e}_{d}\right)$ for $\mathfrak{e} \in \mathcal{E}_{D_{1}}$ is measurable with respect to $\mathscr{F}_{D_{1}}^{+}$. Conditionally on $\mathscr{F}_{D_{1}}^{+}$, the excursions between $\mathfrak{e}_{g}$ and $\mathfrak{e}_{d}$ for $\mathfrak{e} \in \mathcal{E}_{D_{1}}$ are independent Brownian excursions inside $D_{1}$ with law $\mathbb{P}_{D_{1}}^{\mathfrak{e}_{g} \mathfrak{e}_{d}}$. Notice that each excursion inside $D_{1}$ can be associated to at most one $\mathbf{t}_{i}$ : for any excursion $\mathfrak{e} \in \mathcal{E}_{D_{1}}$, write (if it exists) $\mathbf{t}(\mathfrak{e})$ for the time $\mathbf{t}_{i}$ associated, which is the hitting time of $\partial D_{2}$ by the excursion. Set $\mathbf{t}(\mathfrak{e})=\infty$ otherwise. Similarly, we write

$$
\mathbf{s}(\mathfrak{e}):=\inf \left\{t>\mathbf{t}(\mathfrak{e}): \mathfrak{e}_{t} \in \partial D_{1} \cup\left\{z^{\prime}\right\}\right\}
$$

Consequently,

$$
\begin{aligned}
& \sum_{1 \leq i_{1}<\ldots<i_{L}} \prod_{j=1}^{L} \xi_{D_{1}}\left(x, B_{\mathbf{t}_{i_{j}}}, B_{\mathbf{s}_{i_{j}}}\right) 1_{\left\{\mathbf{t}_{i_{L}}<T_{z^{\prime}}\right\}} \\
= & \sum_{\substack{\mathfrak{e}_{D_{1}}^{L}}} \prod_{j=1}^{L} \xi_{D_{1}}\left(x, B_{\mathbf{t}\left(\mathfrak{e}^{j}\right)}, B_{\left.{\mathbf{s}\left(\mathfrak{e}^{j}\right)}\right)}\right) 1_{\left\{\mathbf{t}\left(\mathfrak{e}^{j}\right)<\infty\right\}},
\end{aligned}
$$

where we write $\sum_{\underline{\mathfrak{e}}_{D_{1}}^{L}}$ as a short way for sum over ordered (distinct) excursions $\left(\mathfrak{e}^{1}, \ldots, \mathfrak{e}^{L}\right) \in$ $\left(\mathcal{E}_{D_{1}}\right)^{L} \cdot{ }^{10}$ Apply $(3.2)$ to $D_{1}=D, z=\mathfrak{e}_{g}$, and $z^{\prime}=\mathfrak{e}_{d}$. Notice that in this case, the sum

\footnotetext{
${ }^{10}$ The excursions $\mathfrak{e}^{1}, \ldots, \mathfrak{e}^{L}$ are naturally ordered in terms of their appearances, in particular any set of $L$ distinct excursions appears only once in the sum.
} 
$\sum_{i \geq 1} \ldots$ in (3.2) has at most one term. This yields that for any $\mathfrak{e} \in \mathcal{E}_{D_{1}}$,

$$
\begin{aligned}
& \mathbb{E}_{D}^{z, z^{\prime}}\left[\xi_{D_{1}}\left(x, B_{\mathbf{t}(\mathfrak{e})}, B_{\mathbf{s}(\mathfrak{e})}\right) 1_{\{\mathbf{t}(\mathfrak{e})<\infty\}} \mid \mathscr{F}_{D_{1}}^{+}\right] \\
= & \mathbb{E}_{D_{1}}^{\mathfrak{e}_{g}, \mathfrak{e}_{d}}\left[\sum_{i \geq 1} \xi_{D_{1}}\left(x, B_{\mathbf{t}_{i}}, B_{\mathbf{s}_{i}}\right) 1_{\left\{\mathbf{t}_{i}<T_{\mathfrak{e}_{d}}\right\}}\right] \\
= & \xi_{D_{1}}\left(x, \mathfrak{e}_{g}, \mathfrak{e}_{d}\right) .
\end{aligned}
$$

Hence,

$$
\begin{aligned}
& \mathbb{E}_{D}^{z, z^{\prime}}\left[\sum_{1 \leq i_{1}<\ldots<i_{L}} \prod_{j=1}^{L} \xi_{D_{1}}\left(x, B_{\mathbf{t}_{i_{j}}}, B_{\mathbf{s}_{i_{j}}}\right) 1_{\left\{\mathbf{t}_{i_{L}}<T_{z^{\prime}}\right\}} \mid \mathscr{F}_{D_{1}}^{+}\right] \\
= & \sum_{\underline{\mathfrak{e}}_{D_{1}}^{L}} \prod_{j=1}^{L} \xi_{D_{1}}\left(x, \mathfrak{e}_{g}^{j}, \mathfrak{e}_{d}^{j}\right) .
\end{aligned}
$$

For future use, we observe that the same argument also gives (noting that $\mathbf{s}_{i_{L}}<T_{z^{\prime}}$ ensures $\left.\mathbf{t}_{i_{L}}<T_{z^{\prime}}\right)$

$$
\begin{aligned}
& \mathbb{E}_{D}^{z, z^{\prime}}\left[\sum_{1 \leq i_{1}<\ldots<i_{L}} \prod_{j=1}^{L} \xi_{D_{1}}\left(x, B_{\mathbf{t}_{i_{j}}}, B_{\mathbf{s}_{i_{j}}}\right) 1_{\left\{T_{\partial D_{1}}<\mathbf{t}_{i_{1}}\right\}} 1_{\left\{\mathbf{s}_{i_{L}}<T_{z^{\prime}}\right\}} \mid \mathscr{F}_{D_{1}}^{+}\right] \\
= & \sum_{\underline{e}_{D_{1}}^{L}} \prod_{j=1}^{L} \xi_{D_{1}}\left(x, \mathfrak{e}_{g}^{j}, \mathfrak{e}_{d}^{j}\right) 1_{\left\{\mathfrak{e}_{g}^{1} \neq z, \mathfrak{e}_{d}^{L} \neq z^{\prime}\right\}} .
\end{aligned}
$$

Going back to (3.11), the following proposition is already proved:

Proposition 3.5 Let $D_{1} \subset D$ be nice domains. Let $x \in D_{1}$. Suppose that $z$ and $z^{\prime}$ are distinct nice points of $\bar{D}$, different from $x$, and not in $\partial D_{1} \backslash \mathcal{J}\left(D, D_{1}\right)$. For any $A \in \mathscr{F}_{D_{1}}^{+}$,

$$
\mathbb{Q}_{x, D}^{z, z^{\prime}, \alpha}(A \mid \mathbf{L}=L)=\frac{C_{D, D_{1}}(x)^{-(L-1)}}{\xi_{D}\left(x, z, z^{\prime}\right)} \mathbb{E}_{D}^{z, z^{\prime}}\left[1_{A} \sum_{\mathfrak{e}_{D_{1}}^{L}} \prod_{j=1}^{L} \xi_{D_{1}}\left(x, \mathfrak{e}_{g}^{j}, \mathfrak{e}_{d}^{j}\right)\right],
$$

where as before, $\sum_{\mathfrak{e}_{D_{1}}^{L}}$ means that the sum runs over all ordered (distinct) excursions $\left(\mathfrak{e}^{1}, \ldots, \mathfrak{e}^{L}\right) \in\left(\mathcal{E}_{D_{1}}\right)^{L}$. In particular, we have

$$
\mathbb{E}_{D}^{z, z^{\prime}}\left[\sum_{\underline{\mathfrak{e}}_{D_{1}}^{L}} \prod_{j=1}^{L} \xi_{D_{1}}\left(x, \mathfrak{e}_{g}^{j}, \mathfrak{e}_{d}^{j}\right)\right]=\xi_{D}\left(x, z, z^{\prime}\right) C_{D, D_{1}}(x)^{L-1} .
$$

Recall that $C_{D, D_{1}}(x)=C_{D_{1}}(x)-C_{D}(x)$ by (2.15). Since $\mathbf{L}-1$ is by construction a Poisson random variable with parameter $\alpha \nu_{D}(x, x)\left(T_{\partial D_{1}}<T_{x}\right)=\alpha C_{D, D_{1}}(x)$, we get the following corollary. 
Corollary 3.6 With the notation and assumptions of Proposition 3.5,

$$
\left.\frac{\mathrm{d} \mathbb{Q}_{x, D}^{z, z^{\prime}, \alpha}}{\mathrm{d} \mathbb{P}_{D}^{z, z^{\prime}}}\right|_{\mathscr{F}_{D_{1}}^{+}}=\frac{M_{D_{1}}(x, \alpha)}{\mathrm{e}^{-\alpha C_{D}(x)} \xi_{D}\left(x, z, z^{\prime}\right)},
$$

where

$$
M_{D_{1}}(x, \alpha):=\mathrm{e}^{-\alpha C_{D_{1}}(x)} \sum_{L \geq 1} \frac{\alpha^{L-1}}{(L-1) !} \sum_{\underline{\mathfrak{e}}_{D_{1}}^{L}} \prod_{j=1}^{L} \xi_{D_{1}}\left(x, \mathfrak{e}_{g}^{j}, \mathfrak{e}_{d}^{j}\right) .
$$

By definition, $M_{D}(x, \alpha)=\mathrm{e}^{-\alpha C_{D}(x)} \xi_{D}\left(x, z, z^{\prime}\right)$ under $\mathbb{P}_{D}^{z, z^{\prime}}$.

Corollary 3.7 With the same notation and assumptions of Proposition 3.5. Let $D_{2} \subset D_{1}$ be another nice domain such that $x \in D_{2}$ and that $z, z^{\prime}$ do not lie in $\partial D_{2} \backslash \mathcal{J}\left(D, D_{2}\right) .{ }^{11}$ Then

$$
\mathbb{E}_{D}^{z, z^{\prime}}\left[M_{D_{2}}(x, \alpha) \mid \mathscr{F}_{D_{1}}^{+}\right]=M_{D_{1}}(x, \alpha)
$$

Proof of Corollary 3.7. Let $C \in \mathscr{F}_{D_{1}}^{+}$. Then $C \in \mathscr{F}_{D_{2}}^{+}$, hence in view of Corollary 3.6, we have

$$
\mathbb{E}_{D}^{z, z^{\prime}}\left[1_{C} \frac{M_{D_{2}}(x, \alpha)}{M_{D}(x, \alpha)}\right]=\mathbb{Q}_{x, D}^{z, z^{\prime}, \alpha}(C)=\mathbb{E}_{D}^{z, z^{\prime}}\left[1_{C} \frac{M_{D_{1}}(x, \alpha)}{M_{D}(x, \alpha)}\right]
$$

which implies Corollary 3.7.

The rest of this section is devoted to Proposition 3.9, which controls the conditional expectation of $M_{D_{2}}(\cdot, \alpha)$ under $\mathbb{Q}_{x, D}^{z, z^{\prime}, \alpha}$, and is also the main technical tool in the proof of the forthcoming Proposition 4.2. At first we compute the expectation of $\sum_{\underline{\mathfrak{e}}_{D_{1}}^{L}} \prod_{j=1}^{L} \xi_{D_{1}}\left(x, \mathfrak{e}_{g}^{j}, \mathfrak{e}_{d}^{j}\right) 1_{\left\{\mathfrak{e}_{g}^{1} \neq z, \mathfrak{e}_{d}^{L} \neq z^{\prime}\right\}}$ under different measures:

Lemma 3.8 Let $x \in D_{1}$. Suppose that $z, z^{\prime}$ are distinct nice points of $\bar{D}$, different from $x$, and do not belong to $\partial D_{1} \backslash \mathcal{J}\left(D, D_{1}\right)$. We take the convention that $H_{D_{1}}(x, y)=0$ if

\footnotetext{
${ }^{11}$ It is elementary to check that if $z \notin \partial D_{1} \backslash \mathcal{J}\left(D, D_{1}\right)$, then saying $z \notin \partial D_{2} \backslash \mathcal{J}\left(D, D_{2}\right)$ and saying $z \notin \partial D_{2} \backslash \mathcal{J}\left(D_{1}, D_{2}\right)$ are equivalent.
} 
$y \notin \bar{D}_{1}$. We have the following equalities: For all $L \geq 1$,

$$
\begin{array}{r}
\frac{C_{D, D_{1}}(x)^{-(L-1)}}{\xi_{D}\left(x, z, z^{\prime}\right)} \mathbb{E}_{D}^{z, z^{\prime}}\left[\sum_{\mathfrak{e}_{D_{1}}^{L}} \prod_{j=1}^{L} \xi_{D_{1}}\left(x, \mathfrak{e}_{g}^{j}, \mathfrak{e}_{d}^{j}\right) 1_{\left\{\mathfrak{e}_{g}^{1} \neq z, \mathfrak{e}_{d}^{L} \neq z^{\prime}\right\}}\right] \\
=\left(1-\frac{H_{D_{1}}(x, z)}{H_{D}(x, z)}\right)\left(1-\frac{H_{D_{1}}\left(x, z^{\prime}\right)}{H_{D}\left(x, z^{\prime}\right)}\right), \\
\mathbb{E}_{D}^{z, x}\left[\sum_{\mathfrak{e}_{D_{1}}^{L}} \prod_{j=1}^{L} \xi_{D_{1}}\left(x, \mathfrak{e}_{g}^{j}, \mathfrak{e}_{d}^{j}\right) 1_{\left\{\mathfrak{e}_{g}^{1} \neq z, \mathfrak{e}_{d}^{L} \neq x\right\}}\right]=\left(1-\frac{H_{D_{1}}(x, z)}{H_{D}(x, z)}\right) C_{D, D_{1}}(x)^{L}, \\
\nu_{D}(x, x)\left[\sum_{\underline{\mathfrak{e}}_{D_{1}}^{L}} \prod_{j=1}^{L} \xi_{D_{1}}\left(x, \mathfrak{e}_{g}^{j}, \mathfrak{e}_{d}^{j}\right) 1_{\left\{\mathfrak{e}_{g}^{1} \neq x, \mathfrak{e}_{d}^{L} \neq x\right\}}\right]=C_{D, D_{1}}(x)^{L+1},
\end{array}
$$

where as before, $\sum_{\underline{\underline{e}}_{D_{1}}^{L}}$ means that the sum runs over all ordered (distinct) excursions $\left(\mathfrak{e}^{1}, \ldots, \mathfrak{e}^{L}\right) \in\left(\mathcal{E}_{D_{1}}\right)^{L} ;$ if furthermore, $z \in D$, then

$$
\begin{aligned}
& C_{D, D_{1}}(x)^{-(L-1)} \nu_{D}(z, z)\left[\sum_{\mathfrak{\varepsilon}_{D_{1}}^{L}} \prod_{j=1}^{L} \xi_{D_{1}}\left(x, \mathfrak{e}_{g}^{j}, \mathfrak{e}_{d}^{j}\right) 1_{\left\{\mathfrak{e}_{g}^{1} \neq z, \mathfrak{e}_{d}^{L} \neq z\right\}}\right] \\
= & (2 \pi)^{2}\left(H_{D}(x, z)-H_{D_{1}}(x, z)\right)^{2} .
\end{aligned}
$$

Proof of Lemma 3.8. Let $A$ be the event that, under $\mathbb{Q}_{x, D}^{z, z^{\prime}, \alpha}$, the first part of the trajectory from $z$ to $x$ hits $\partial D_{1}$, and so does the last part from $x$ to $z^{\prime}$.

We have by Lemma 2.2 (ii),

$$
\begin{aligned}
\mathbb{Q}_{x, D}^{z, z^{\prime}, \alpha}(A \mid \mathbf{L}=L) & =\mathbb{P}_{D}^{z, x}\left(T_{\partial D_{1}}<T_{x}\right) \mathbb{P}_{D}^{x, z^{\prime}}\left(T_{\partial D_{1}}<T_{z^{\prime}}\right) \\
& =\left(1-\frac{H_{D_{1}}(x, z)}{H_{D}(x, z)}\right)\left(1-\frac{H_{D_{1}}\left(x, z^{\prime}\right)}{H_{D}\left(x, z^{\prime}\right)}\right) .
\end{aligned}
$$

Let $D_{2}$ be a nice domain such that $x \in D_{2}, \overline{D_{2}} \subset D_{1}$ and that $z, z^{\prime} \notin D_{2}$. Observe that for all $1 \leq i_{1}<\ldots<i_{L}, A \cap\left\{\mathbf{L}=L, \mathbf{i}_{1}=i_{1}, \mathbf{i}_{L}=i_{L}\right\}=A\left(i_{1}, i_{L}\right) \cap\left\{\mathbf{L}=L, \mathbf{i}_{1}=\right.$ $\left.i_{1}, \mathbf{i}_{L}=i_{L}\right\}$, with $A\left(i_{1}, i_{L}\right):=\left\{T_{\partial D_{1}}<\mathbf{t}_{i_{1}}, B_{\mathbf{s}_{i_{L}}} \neq z^{\prime}\right\} \in \mathscr{F}_{D_{1}}^{+}$. Then

$$
\begin{aligned}
& \mathbb{Q}_{x, D}^{z, z^{\prime}, \alpha}(A \mid \mathbf{L}=L) \\
= & \sum_{1 \leq i_{1}<\ldots<i_{L}} \mathbb{Q}_{x, D}^{z, z^{\prime}, \alpha}\left(A\left(i_{1}, i_{L}\right), \mathbf{i}_{j}=i_{j}, 1 \leq j \leq L \mid \mathbf{L}=L\right) \\
= & \frac{C_{D, D_{1}}(x)^{-(L-1)}}{\xi_{D}\left(x, z, z^{\prime}\right)} \mathbb{E}_{D}^{z, z^{\prime}}\left(\sum_{1 \leq i_{1}<\ldots<i_{L}} 1_{\left\{T_{\partial D_{1}}<\mathbf{t}_{i_{1}}, B_{\mathbf{s}_{L}} \neq z^{\prime}\right\}} \prod_{j=1}^{L} \xi_{D_{1}}\left(x, y_{j}, y_{j}^{\prime}\right)\right),
\end{aligned}
$$


the last identity being a consequence of (3.10). Applying (3.13) gives

$$
\begin{aligned}
& \mathbb{Q}_{x, D}^{z, z^{\prime}, \alpha}(A \mid \mathbf{L}=L) \\
= & \frac{C_{D, D_{1}}(x)^{-(L-1)}}{\xi_{D}\left(x, z, z^{\prime}\right)} \mathbb{E}_{D}^{z, z^{\prime}}\left[\sum_{\mathfrak{e}_{D_{1}}^{L}} \prod_{j=1}^{L} \xi_{D_{1}}\left(x, \mathfrak{e}_{g}^{j}, \mathfrak{e}_{d}^{j}\right) 1_{\left\{\mathfrak{e}_{g}^{1} \neq z, \mathfrak{e}_{d}^{L} \neq z^{\prime}\right\}}\right],
\end{aligned}
$$

which is the same expression as on the left-hand side of (3.16). Together with (3.20), this yields (3.17).

Recall the definition of $C_{D, D_{1}}(x)$ in $(2.15)$ and that $\lim _{z^{\prime} \rightarrow x}\left(G_{D}\left(x, z^{\prime}\right)-G_{D_{1}}\left(x, z^{\prime}\right)\right)=$ $C_{D, D_{1}}(x)$ as proved in $(2.16)$. It follows that

$$
\xi_{D}\left(x, z, z^{\prime}\right)\left(1-\frac{H_{D_{1}}\left(x, z^{\prime}\right)}{H_{D}\left(x, z^{\prime}\right)}\right) \rightarrow C_{D, D_{1}}(x), \quad \text { as } z^{\prime} \rightarrow x .
$$

Then making $z^{\prime} \rightarrow x$ in (3.16) implies (3.17).

Notice that as $z \rightarrow x, 1-\frac{H_{D_{1}}(x, z)}{H_{D}(x, z)}=\frac{G_{D}(x, z)-G_{D_{1}}(x, z)}{G_{D}(x, z)} \sim \frac{C_{D, D_{1}}(x)}{\log 1 /|x-z|}$ by (2.1). Using (2.13), making $z \rightarrow x$ in (3.17) gives (3.18).

Recall that $H_{D}\left(y, y^{\prime}\right)=G_{D}\left(y, y^{\prime}\right) / 2 \pi$ when $y, y^{\prime}$ are in $D$. When $z \in D \backslash \partial D_{1}$, making $z^{\prime} \rightarrow z \neq x$ in (3.16), and using (2.1) and (2.13) give (3.19).

Let us go back to the probability $\mathbb{Q}_{x, D}^{z, z^{\prime}, \alpha}$ for $z \neq z^{\prime}$ which are different from $x$. For any nice domains $S \subset D_{1} \subset D$, such that $x \in S$, we introduce $\mathcal{E}_{x, D_{1}, S}$, the set of excursions away from $D_{1}^{c} \cup\{z\} \cup\left\{z^{\prime}\right\} \cup\{x\}$ (in the sense of (2.6)) but excluding all loops at $x$ that lie in $S .^{12}$

For any nice domain set $S \subset D_{1}$ which contains $x$, any nice domain $D_{2} \subset D_{1}$, and any $u \in D_{2} \backslash\{x\}$, let

$$
M_{x, D_{1}, S, D_{2}}(u, \alpha):=\mathrm{e}^{-\alpha C_{D_{1}}(u)} \sum_{L=1}^{\infty} \frac{\alpha^{L-1}}{(L-1) !} \sum_{\substack{\mathfrak{e}_{x, D_{1}, S}^{L} \\ j}}^{L} \prod_{j=1}^{L} \xi_{x, D_{1}, S, D_{2}}\left(u, \mathfrak{e}_{g}^{j}, \mathfrak{e}_{d}^{j}\right)
$$

where the sum $\sum_{\underline{\mathfrak{e}}_{x, D_{1}, S}^{L}}$ runs over all ordered (distinct) excursions $\left(\mathfrak{e}^{1}, \ldots, \mathfrak{e}^{L}\right)$ in $\left(\mathcal{E}_{x, D_{1}, S}\right)^{L}$

\footnotetext{
${ }^{12}$ In words, $\mathcal{E}_{x, D_{1}, S}$ stands for the set of excursions in $\mathcal{E}_{D_{1}}$ that do not hit $x$, to which we also add: (a) the excursions from $\partial D_{1}$ to $x$ (if they exist); (b) the excursions from $x$ to $\partial D_{1}$ (if they exist); (c) loops at $x$ which lie in $D_{1}$ and hit $\partial S$; (d) if $z \in D_{1}$, the path from $z$ to $x$ if it does not hit $\partial D_{1}$; (e) if $z^{\prime} \in D_{1}$, the path from $x$ to $z^{\prime}$ if it does not hit $\partial D_{1}$.
} 
and

$$
\begin{aligned}
\xi_{x, D_{1}, S, D_{2}}\left(u, \mathfrak{e}_{g}, \mathfrak{e}_{d}\right):= & \\
\begin{cases}\frac{\xi_{D_{1}}\left(u, \mathfrak{e}_{g}, \mathfrak{e}_{d}\right),}{(2 \pi)^{2}\left(H_{D_{1}}(u, x)-H_{D_{2}}(u, x)\right)^{2}} & \text { if } \mathfrak{e}_{g}, \mathfrak{e}_{d} \in \partial D_{1}, \\
\frac{2 \pi\left(H_{D_{1}}\left(u, \mathfrak{e}_{g}\right)-H_{D_{2}}\left(u, \mathfrak{e}_{g}\right)\right)\left(H_{D_{1}}\left(u, \mathfrak{e}_{d}\right)-H_{D_{2}}\left(u, \mathfrak{e}_{d}\right)\right)}{H_{D_{1}}\left(\mathfrak{e}_{g}, \mathfrak{e}_{d}\right)}, & \text { if } \mathfrak{e}_{g}=\mathfrak{e}_{d}=x,\end{cases} & \text { otherwise. }
\end{aligned}
$$

Considering (3.21), and the inequality $\sum_{j \geq 0} \frac{\lambda^{j}}{(j !)^{2}} \leq \mathrm{e}^{2 \sqrt{\lambda}}$ for any $\lambda \geq 0$, we have the following bound:

$$
M_{x, D_{1}, S, D_{2}}(u, \alpha) \leq \Upsilon_{x, D_{1}, S, D_{2}}(u) \mathrm{e}^{-\alpha C_{D_{1}}(u)} \mathrm{e}^{2 \sqrt{\alpha \Upsilon_{x, D_{1}, S, D_{2}}(u)}}
$$

where

$$
\Upsilon_{x, D_{1}, S, D_{2}}(u):=\sum_{\mathfrak{e} \in \mathcal{E}_{x, D_{1}, S}} \xi_{x, D_{1}, S, D_{2}}\left(u, \mathfrak{e}_{g}, \mathfrak{e}_{d}\right) .
$$

Proposition 3.9 Let $S \subset D_{1} \subset D$ be nice domains such that $x \in S$. Let $D_{2} \subset D_{1}$ be a nice domain satisfying $\partial D_{2} \subset S^{c}$. Suppose that $z$ and $z^{\prime}$ are distinct nice points of $\bar{D}$, different from $x$, which belong neither to $\partial D_{1} \backslash \mathcal{J}\left(D, D_{1}\right)$ nor to $\partial D_{2} \backslash \mathcal{J}\left(D_{1}, D_{2}\right)$. Let $u \in D_{2} \backslash\{x\}$. Define

$$
\widetilde{M}_{x, D_{2}}(u, \alpha):=\mathrm{e}^{-\alpha C_{D_{2}}(u)} \sum_{L=1}^{\infty} \frac{\alpha^{L-1}}{(L-1) !} \sum_{\underline{\mathfrak{e}}_{D_{2}}^{L}} \prod_{j=1}^{L} \xi_{D_{2}}\left(u, \mathfrak{e}_{g}^{j}, \mathfrak{e}_{d}^{j}\right) 1_{\{x \notin \mathfrak{e} j\}} .
$$

We have, with the notation of (3.21) and (3.24),

$$
\begin{aligned}
& \mathbb{E}_{\mathbb{Q}_{x, D}^{z, z^{\prime}, \alpha}}\left[\widetilde{M}_{x, D_{2}}(u, \alpha) \mid\left(\mathfrak{e}_{g}, \mathfrak{e}_{d}\right)_{\left.\mathfrak{e} \in \mathcal{E}_{x, D_{1}, S}\right]}\right] \\
= & M_{x, D_{1}, S, D_{2}}(u, \alpha) \\
\leq & \Upsilon_{x, D_{1}, S, D_{2}}(u) \mathrm{e}^{-\alpha C_{D_{1}}(u)} \mathrm{e}^{2 \sqrt{\alpha \Upsilon_{x, D_{1}, S, D_{2}}(u)}} .
\end{aligned}
$$

Proof. Any element of $\mathcal{E}_{D_{2}}$ that does not hit $x$, necessarily hits $\partial D_{2}$, and lies (except, possibly, for the starting or the ending point) in $D_{1} \backslash\left\{x, z, z^{\prime}\right\}$, and is thus contained in an excursion, say $\mathfrak{e}$, away from $D_{1}^{c} \cup\left\{x, z, z^{\prime}\right\}$; $\mathfrak{e}$ cannot be a loop at $x$ lying in $S$ because $\partial D_{2} \subset S^{c}$. In other words, $\mathfrak{e} \in \mathcal{E}_{x, D_{1}, S}$. It follows that

$$
\begin{aligned}
& \widetilde{M}_{x, D_{2}}(u, \alpha) \\
= & \mathrm{e}^{-\alpha C_{D_{2}}(u)} \sum_{L=1}^{\infty} \frac{\alpha^{L-1}}{(L-1) !} \sum_{K=1}^{L} \sum_{\underline{\underline{L}}_{x, D_{1}, S}^{K}} \sum_{\ell_{1}+\ldots+\ell_{K}=L, \ell_{j} \geq 1, \forall j \leq K} \prod_{j=1}^{K} \Upsilon\left(\mathfrak{e}^{j}, \ell_{j}\right),
\end{aligned}
$$


where as before, the sum $\sum_{\mathfrak{e}_{x, D_{1}, S}^{K}}$ runs over ordered (distinct) all excursions $\left(\mathfrak{e}^{1}, \ldots, \mathfrak{e}^{K}\right) \in$ $\left(\mathcal{E}_{x, D_{1}, S}\right)^{K}$, and $\Upsilon\left(\mathfrak{e}^{j}, \ell_{j}\right)$ is the sum, over all $\ell_{j}$ distinct excursions $\mathfrak{e}^{j, 1}, \ldots, \mathfrak{e}^{j, \ell_{j}}$ in $\mathcal{E}_{D_{2}}$ which do not hit $x$ and which is contained in the excursion $\mathfrak{e}^{j}$, of the products $\prod_{i=1}^{\ell_{j}} \xi_{D_{2}}\left(u, \mathfrak{e}_{g}^{j, i}, \mathfrak{e}_{d}^{j, i}\right)$.

Notice that conditionally on $\left(\mathfrak{e}_{g}, \mathfrak{e}_{d}\right)_{\mathfrak{e} \in \mathcal{E}_{x, D_{1}, S}} \in\left(\mathbb{C}^{2}\right)^{\mathcal{E}_{x, D_{1}, S}}$, the family $\left(\Upsilon\left(\mathfrak{e}^{j}, \ell_{j}\right)\right)_{1 \leq j \leq K}$ for distinct $\mathfrak{e}^{1}, \ldots, \mathfrak{e}^{K} \in \mathcal{E}_{x, D_{1}, S}$, is independent. We claim that

$$
\mathbb{E}_{\mathbb{Q}_{x, D}^{z, z^{\prime}, \alpha}}\left[\Upsilon\left(\mathfrak{e}^{j}, \ell_{j}\right) \mid\left(\mathfrak{e}_{g}, \mathfrak{e}_{d}\right)_{\mathfrak{e} \in \mathcal{E}_{x, D_{1}, S}}\right]=C_{D_{1}, D_{2}}(u)^{\ell_{j}-1} \xi_{x, D_{1}, S, D_{2}}\left(u, \mathfrak{e}_{g}^{j}, \mathfrak{e}_{d}^{j}\right) .
$$

Indeed by definition of $\mathcal{E}_{x, D_{1}, S}$, the excursion $\mathfrak{e}^{j}$ can be an excursion from $\partial D_{1}$ to $\partial D_{1}$ which does not hit $x$, or from $\partial D_{1}$ to $x$ or from $x$ to $\partial D_{1}$, or a loop at $x$ in $D_{1}$ conditioned to hit $\partial S$. Let us check (3.27) for each of these four cases. Write $Q_{(3.27)}$ for the conditional expectation term on the left-hand side of (3.27).

If $\mathfrak{e}^{j}$ is an excursion from $\partial D_{1}$ to $\partial D_{1}$ which does not hit $x$, then $\mathfrak{e}_{g}^{j} \in \partial D_{1}, \mathfrak{e}_{d}^{j} \in \partial D_{1}$ and none of $\mathfrak{e}^{j, 1}, \ldots, \mathfrak{e}^{j, \ell_{j}}$ hits $x$. By the Markov property,

$$
Q_{(3.27)}=\mathbb{E}_{D_{1}}^{\mathfrak{s}_{g}^{j}, \mathfrak{e}_{d}^{j}}\left[\sum_{\substack{\ell_{\ell} \\ \underline{\varepsilon}_{D_{2}}}} \prod_{j=1}^{\ell_{j}} \xi_{D_{2}}\left(u, \mathfrak{e}_{g}^{j}, \mathfrak{e}_{d}^{j}\right)\right]=C_{D_{1}, D_{2}}(u)^{\ell_{j}-1} \xi_{D_{1}}\left(u, \mathfrak{e}_{g}^{j}, \mathfrak{e}_{d}^{j}\right),
$$

by using (3.14) for the second equality. This implies (3.27) as $\xi_{x, D_{1}, S, D_{2}}\left(u, \mathfrak{e}_{g}^{j}, \mathfrak{e}_{d}^{j}\right)=$ $\xi_{D_{1}}\left(u, \mathfrak{e}_{g}^{j}, \mathfrak{e}_{d}^{j}\right)$ in this case.

If $\mathfrak{e}^{j}$ is an excursion from $\partial D_{1}$ to $x$, then $\mathfrak{e}_{g}^{j} \in \partial D_{1}$ and $\mathfrak{e}_{d}^{j}=x$. By the Markov property and (3.16),

$$
Q_{(3.27)}=C_{D_{1}, D_{2}}(u)^{\ell_{j}-1} \xi_{D_{1}}\left(u, \mathfrak{e}_{g}^{j}, \mathfrak{e}_{d}^{j}\right)\left(1-\frac{H_{D_{2}}\left(u, \mathfrak{e}_{g}^{j}\right)}{H_{D_{1}}\left(u, \mathfrak{e}_{g}^{j}\right)}\right)\left(1-\frac{H_{D_{2}}\left(u, \mathfrak{e}_{d}^{j}\right)}{H_{D_{1}}\left(u, \mathfrak{e}_{d}^{j}\right)}\right),
$$

which yields (3.27) by the definition of $\xi_{x, D_{1}, S, D_{2}}\left(u, \mathfrak{e}_{g}^{j}, \mathfrak{e}_{d}^{j}\right)$ (recalling that $\mathfrak{e}_{g}^{j} \neq x$ and $\mathfrak{e}_{d}^{j}=x$ in this case). The case when $\mathfrak{e}^{j}$ is an excursion from $x$ to $\partial D_{1}$ follows from the same way.

Finally for the case when $\mathfrak{e}^{j}$ is a loop at $x$ in $D_{1}$ conditioned to hit $\partial S, \mathfrak{e}_{g}^{j}=\mathfrak{e}_{d}^{j}=x$, so

$$
\begin{aligned}
Q_{(3.27)} & =\nu_{D_{1}}(x, x)\left[\sum_{\substack{\ell_{j} \\
\underline{\ell}_{D_{2}}}} \prod_{j=1}^{\ell_{j}} \xi_{D_{2}}\left(u, \mathfrak{e}_{g}^{j}, \mathfrak{e}_{d}^{j}\right) 1_{\left\{\mathfrak{c}_{g}^{1} \neq x, \mathfrak{e}_{d}^{\ell_{j}} \neq x\right\}} \mid T_{\partial S}<T_{x}\right] \\
& =\frac{\nu_{D_{1}}(x, x)\left[\sum_{\underline{\varepsilon}_{D_{2}}^{\ell_{j}}} \prod_{j=1}^{\ell_{j}} \xi_{D_{2}}\left(u, \mathfrak{e}_{g}^{j}, \mathfrak{e}_{d}^{j}\right) 1_{\left\{\mathfrak{e}_{g}^{1} \neq x, \mathfrak{e}_{d}^{\ell_{j}} \neq x\right\}}\right]}{\nu_{D_{1}}(x, x)\left(T_{\partial S}<T_{x}\right)} .
\end{aligned}
$$

Recall from (2.15) that $\nu_{D_{1}}(x, x)\left(T_{\partial S}<T_{x}\right)=C_{D_{1}, S}(x)$. Using (3.19) completes the proof of (3.27). 
By $(3.27), \mathbb{E}_{\mathbb{Q}_{x, D}^{z, z^{\prime}, \alpha}}\left[\widetilde{M}_{x, D_{2}}(u, \alpha) \mid\left(\mathfrak{e}_{g}, \mathfrak{e}_{d}\right)_{\mathfrak{e} \in \mathcal{E}_{x, D_{1}, S}}\right]$ is given by

$$
\begin{aligned}
\mathrm{e}^{-\alpha C_{D_{2}}(u)} \sum_{L=1}^{\infty} \frac{\alpha^{L-1}}{(L-1) !} & \sum_{K=1}^{L} C_{D_{1}, D_{2}}(u)^{L-K} \times \\
& \left\{\sum_{\underline{\mathfrak{e}}_{x, D_{1}, S}^{K}} \sum_{\ell_{1}+\ldots+\ell_{K}=L, \ell_{j} \geq 1, \forall j \leq K} \prod_{j=1}^{K} \xi_{x, D_{1}, S, D_{2}}\left(u, \mathfrak{e}_{g}^{j}, \mathfrak{e}_{d}^{j}\right)\right\} .
\end{aligned}
$$

The $\operatorname{sum} \sum_{L=1}^{\infty} \ldots$ is also

$$
\begin{aligned}
& \sum_{L=1}^{\infty} \frac{\alpha^{L-1}}{(L-1) !} \sum_{K=1}^{L} C_{D_{1}, D_{2}}(u)^{L-K} \sum_{\underline{\mathfrak{e}}_{x, D_{1}, S}^{K}}\left(\begin{array}{c}
L-1 \\
K-1
\end{array}\right) \prod_{j=1}^{K} \xi_{x, D_{1}, S, D_{2}}\left(u, \mathfrak{e}_{g}^{j}, \mathfrak{e}_{d}^{j}\right) \\
= & \sum_{K=1}^{\infty} \sum_{\underline{\mathfrak{e}}_{x, D_{1}, S}^{K}} \prod_{j=1}^{K} \xi_{x, D_{1}, S, D_{2}}\left(u, \mathfrak{e}_{g}^{j}, \mathfrak{e}_{d}^{j}\right) \sum_{L=K}^{\infty} \frac{\alpha^{L-1}}{(K-1) !(L-K) !} C_{D_{1}, D_{2}}(u)^{L-K} .
\end{aligned}
$$

Since

$$
\sum_{L=K}^{\infty} \frac{\alpha^{L-1}}{(K-1) !(L-K) !} C_{D_{1}, D_{2}}(u)^{L-K}=\frac{\alpha^{K-1}}{(K-1) !} \mathrm{e}^{\alpha C_{D_{1}, D_{2}}(u)}
$$

and $C_{D_{1}, D_{2}}(u)=C_{D_{2}}(u)-C_{D_{1}}(u)$, we get (3.25), which, in turn (in view of (3.23)), yields $(3.26)$.

\section{Construction of the measure $\mathcal{M}_{\infty}^{\alpha}$}

Let $D$ be a simply connected nice domain. Let $z$ and $z^{\prime}$ be distinct nice points of $\bar{D}$. For $n \geq 0$, we let $\mathscr{D}_{n}$ be the set of the connected components of $D$ minus the grid of mesh size $2^{-n}$. We choose $\left(\mathscr{D}_{n}, n \geq 0\right)$ such that $z$ and $z^{\prime}$ never lie in any of the grid. We will abusively call an element of $\mathscr{D}_{n}$ a square (it is not necessarily a square near the boundary of $D$ ). We say that $x$ is suitable if $x$ is in some square $D_{n} \in \mathscr{D}_{n}$ at any level $n$, and is different from $z$ and $z^{\prime}$. The set of suitable points has full Lebesgue measure.

For any suitable $x$, we let $D_{n}^{(x)}$ be the square in $\mathscr{D}_{n}$ that contains $x$. We let $\mathscr{F}_{\mathscr{D}_{n}}$ be the sigma-algebra generated by the starting and return points of all excursions inside some square $D_{n}$ together with the order of their appearances. Then $\left(\mathscr{F}_{\mathscr{D}_{n}}\right)_{n \geq 0}$ is a filtration, and $\sigma\left(\cup_{n=0}^{\infty} \mathscr{F}_{\mathscr{D}_{n}}\right)$ coincides with $\sigma\left(B_{t}, t \in\left[0, T_{z^{\prime}}\right]\right)$. ${ }^{13}$ We define for any Borel set $A$,

$$
\mathcal{M}_{\mathscr{D}_{n}}^{\alpha}(A):=\int_{A} M_{D_{n}^{(x)}}(x, \alpha) \mathrm{d} x
$$

\footnotetext{
${ }^{13}$ To identify the two sigma-algebras, note that by continuity, it suffices to show that for any disc $\mathcal{B}(x, r) \subset D$ (where $r>0$ is rational, and $x$ is with rational coordinates), the duration and the exiting position of Brownian motion starting at any $y \in \mathcal{B}(x, r)$ and killed upon exiting from $\mathcal{B}(x, r)$ are mea-
} 
where $M_{D_{n}^{(x)}}(x, \alpha)$ is defined in (3.15) and $M_{D_{n}^{(x)}}(x, \alpha):=0$ if $x$ is not suitable.

Note that $\mathcal{M}_{\mathscr{D}_{n}}^{\alpha}(A)$ is $\mathscr{F}_{\mathscr{D}_{n}}$-adapted. For any suitable $x, \mathscr{F}_{\mathscr{D}_{n}} \subset \mathscr{F}_{D_{n}^{(x)}}^{+}$(defined in Notation 3.4 for the latter), so we can use Corollary 3.7 and integrate over $A$ to yield that $\left(\mathcal{M}_{\mathscr{D}_{n}}^{\alpha}(A)\right)_{n \geq 0}$ is an $\left(\mathscr{F}_{\mathscr{D}_{n}}\right)$-martingale under $\mathbb{P}_{D}^{z, z^{\prime}}$. Consequently, the following limit exists:

$$
\mathcal{M}_{\infty}^{\alpha}(A):=\lim _{n \rightarrow+\infty} \mathcal{M}_{\mathscr{D}_{n}}^{\alpha}(A) \in[0, \infty), \quad \mathbb{P}_{D}^{z, z^{\prime}} \text {-a.s. }
$$

Theorem 4.1 Let $D$ be a simply connected nice domain, and $z$ and $z^{\prime}$ be distinct nice points of $\bar{D}$. Fix $0 \leq \alpha<2$ and a Borel set $A$ of $D$. Under $\mathbb{P}_{D}^{z, z^{\prime}}$, the martingale $\left(\mathcal{M}_{\mathscr{D}_{n}}^{\alpha}(A)\right)_{n \geq 0}$ converges in $L^{1}$ to $\mathcal{M}_{\infty}^{\alpha}(A)$.

In order to prove Theorem 4.1, we first remark that by Corollary 3.6, the (finite) measure $\int_{A} \mathbb{Q}_{x, D}^{z, z^{\prime}, \alpha}(\bullet) M_{D}(x, \alpha) \mathrm{d} x$ has Radon-Nikodym derivative $\mathcal{M}_{\mathscr{D}_{n}}^{\alpha}(A)$ with respect to $\mathbb{P}_{D}^{z, z^{\prime}}$ on $\mathscr{F}_{\mathscr{D}_{n}}$. We assume without loss of generality that $A$ has a positive Lebesgue measure. Let

$$
Q_{A}^{z, z^{\prime}, \alpha}(\bullet):=\frac{1}{\int_{A} M_{D}(x, \alpha) \mathrm{d} x} \int_{A} \mathbb{Q}_{x, D}^{z, z^{\prime}, \alpha}(\bullet) M_{D}(x, \alpha) \mathrm{d} x
$$

be the normalized probability measure defined on $\sigma\left(\cup_{n=0}^{\infty} \mathscr{F}_{\mathscr{D}_{n}}\right)$.

By an elementary fact (see Durrett [6], Theorem 5.3.3), $\mathcal{M}_{\mathscr{D}_{n}}^{\alpha}(A)$ converges in $L^{1}\left(\mathbb{P}_{D}^{z, z^{\prime}}\right)$ if and only if $\mathcal{M}_{\infty}^{\alpha}(A)<\infty, Q_{A}^{z, z^{\prime}, \alpha}$-a.s., where $\mathcal{M}_{\infty}^{\alpha}(A)$ is the $Q_{A}^{z, z^{\prime}, \alpha}$-a.s. limit of $\mathcal{M}_{\mathscr{D}_{n}}^{\alpha}(A)$, which exists since $1 / \mathcal{M}_{\mathscr{D}_{n}}^{\alpha}(A)$ is a nonnegative supermartingale under $Q_{A}^{z, z^{\prime}, \alpha}$.

Then Theorem 4.1 follows from the next proposition.

Proposition 4.2 Let $D$ be a simply connected nice domain, and $z$ and $z^{\prime}$ be distinct nice points of $\bar{D}$. Fix $0 \leq \alpha<2$. Then

$$
\mathbb{Q}_{x, D}^{z, z^{\prime}, \alpha}\left(\mathcal{M}_{\infty}^{\alpha}\left(\mathbb{R}^{2}\right)<\infty\right)=1, \quad \text { Lebesgue-a.e. } x \in D .
$$

As a consequence of Theorem 4.1, we have

Corollary 4.3 Under the assumption of Proposition 4.2, we may define a random finite measure $\mathfrak{m}$ on the Borel sets such that $\mathbb{P}_{D}^{z, z^{\prime}}$-a.s., $\mathfrak{m}$ is the weak limit of $\mathcal{M}_{\mathscr{D}_{n}}^{\alpha}$. Moreover, for any rectangle $A, \mathfrak{m}(A)=\lim _{n \rightarrow \infty} \mathcal{M}_{\mathscr{D}_{n}}^{\alpha}(A), \mathbb{P}_{D}^{z, z^{\prime}}$-a.s.

surable with respect to $\sigma\left(\cup_{n=0}^{\infty} \mathscr{F}_{\mathscr{D}_{n}}\right)$. This, however, is quite straightforward because by continuity, the number of crossings and their positions between two concentric circles (hence the local time on any circle, hence the duration by integration over local time) are measurable with respect to $\sigma\left(\cup_{n=0}^{\infty} \mathscr{F}_{\mathscr{D}_{n}}\right)$. 
Proof of Corollary 4.3. The argument is routine; we give the details for the sake of completeness.

First, note that the sequence $\left(\mathcal{M}_{\mathscr{D}_{n}}^{\alpha}\right)_{n \geq 0}$ is tight, all the measures being supported in the compact set $\bar{D}$; so we can extract a (random) subsequence, say $(n(k), k \geq 1)$, along which $\mathcal{M}_{\mathscr{D}_{n(k)}}^{\alpha}$ converges weakly to some finite random measure $\mathfrak{m}$.

We may define $M_{\infty}^{\alpha}(A)$ such that $\mathbb{P}_{D}^{z, z^{\prime}}$-a.s., (4.2) simultaneously holds for all rectangles $A$ with rational coordinates. For any $\varepsilon>0$ and rectangle $A$, let $A_{+, \varepsilon}$ be an open rectangle and $A_{-, \varepsilon}$ be a closed rectangle both with rational coordinates such that $A_{-, \varepsilon} \subset \AA \subset \bar{A} \subset$ $A_{+, \varepsilon}$ and $\int_{A_{+, \varepsilon} \backslash A_{-, \varepsilon}} M_{D}(x, \alpha) \mathrm{d} x \leq \varepsilon$. By the $L^{1}$-convergence in Theorem 4.1, for any $\varepsilon>0, \mathbb{E}_{D}^{z, z^{\prime}}\left(M_{\infty}^{\alpha}\left(A_{+, \varepsilon}\right)-M_{\infty}^{\alpha}\left(A_{-, \varepsilon}\right)\right)=\int_{A_{+, \varepsilon} \backslash A_{-, \varepsilon}} M_{D}(x, \alpha) \mathrm{d} x \leq \varepsilon$. It implies that for any rectangle $A$,

$$
\inf _{\varepsilon>0}\left(M_{\infty}^{\alpha}\left(A_{+, \varepsilon}\right)-M_{\infty}^{\alpha}\left(A_{-, \varepsilon}\right)\right)=0 \quad \mathbb{P}_{D}^{z, z^{\prime}} \text {-a.s. }
$$

Consider the event $\mathscr{A}$ on which (4.2) and (4.5) hold for all rectangles $A$ with rational coordinates. By the Portmanteau theorem, for any $\varepsilon>0$, we necessarily have $\mathfrak{m}(\bar{A}) \leq$ $\mathfrak{m}\left(A_{+, \varepsilon}\right) \leq \liminf _{k \rightarrow \infty} \mathcal{M}_{\mathscr{D}_{n(k)}}^{\alpha}\left(A_{+, \varepsilon}\right)=M_{\infty}^{\alpha}\left(A_{+, \varepsilon}\right)$, and similarly, $\mathfrak{m}(\stackrel{\AA}{A}) \geq M_{\infty}^{\alpha}\left(A_{-, \varepsilon}\right)$. Consequently,

$$
\mathfrak{m}(\partial A) \leq \inf _{\varepsilon>0}\left(M_{\infty}^{\alpha}\left(A_{+, \varepsilon}\right)-M_{\infty}^{\alpha}\left(A_{-, \varepsilon}\right)\right)=0
$$

for any rectangle $A$ with rational coordinates on the event $\mathscr{A}$. The Portmanteau theorem implies that $\mathfrak{m}(A)=\lim _{k \rightarrow \infty} \mathcal{M}_{\mathscr{D}_{n(k)}}^{\alpha}(A)=M_{\infty}^{\alpha}(A)$ for any such $A$, which by the monotone class theorem yields the uniqueness of the limit measure $\mathfrak{m}$ on $\mathscr{A}$ and proves the $\mathbb{P}_{D}^{z, z^{\prime}}$-a.s. weak convergence of the sequence $\left(\mathcal{M}_{\mathscr{D}_{n}}^{\alpha}\right)_{n \geq 0}$ to $\mathfrak{m}$. Finally, for any rectangle $A$ (regardless of the rationality of the coordinates of $A$ ), equation (4.5) holds $\mathbb{P}_{D}^{z, z^{\prime}}$-a.s., hence the same reasoning shows that $\mathfrak{m}(A)=\lim _{n \rightarrow \infty} \mathcal{M}_{\mathscr{D}_{n}}^{\alpha}(A), \mathbb{P}_{D}^{z, z^{\prime}}$-a.s.

Definition 4.4 (Definition of $M_{\infty}^{\alpha}$ ) In the sequel, by a slight abuse of notation, we still denote by $M_{\infty}^{\alpha}$ the finite random measure $\mathfrak{m}$ in Corollary 4.3.

Theorem 4.1 implies in particular that $\mathbb{E}_{D}^{z, z^{\prime}}\left(\mathcal{M}_{\infty}^{\alpha}(D)\right)>0$. Furthermore, $\mathbb{P}_{D}^{z, z^{\prime}}$-a.s., $\mathcal{M}_{\infty}^{\alpha}$ is not trivial, see Proposition 5.3.

The rest of this section is devoted to the proof of Proposition 4.2. Firstly we present in Section 4.1 some preliminary estimates under $\mathbb{Q}_{x, D}^{z, z^{\prime}, \alpha}$ by means of elementary properties of $\xi_{D}$; then we give the proof in Section 4.2. 


\subsection{Preliminary estimates}

Let $x \in D$. Let $y, z \in \bar{D}$ be distinct nice points, different from $x$. Recall from (3.1) that $\xi_{D}(x, y, z)=\frac{2 \pi H_{D}(x, y) H_{D}(x, z)}{H_{D}(y, z)}$. By properties of harmonic functions under conformal transformations, we see that $\xi_{D}$ is invariant under conformal transformations: if $D$ and $D^{\prime}$ are two nice domains, $z \neq z^{\prime}$ nice points of $\bar{D}, \Psi: D \rightarrow D^{\prime}$ a conformal transformation such that $\Psi(z)$ and $\Psi\left(z^{\prime}\right)$ are nice points of $\overline{D^{\prime}}$, then

$$
\xi_{D^{\prime}}\left(\Psi(x), \Psi(z), \Psi\left(z^{\prime}\right)\right)=\xi_{D}\left(x, z, z^{\prime}\right) .
$$

Lemma 4.5 Let $D$ be a simply connected nice domain and $z^{\prime} \in \partial D$ be a nice point.

(i) For any $x \in D$, and any nice point $z \in \partial D$ different from $z^{\prime}$, we have

$$
\xi_{D}\left(x, z, z^{\prime}\right) \leq 2
$$

(ii) For any $\delta \in(0,1)$, there exists some positive constant $c_{4}=c_{4}(\delta, D)$ such that for any $x, z \in D$ with $|x-z|>\delta$, we have

$$
\xi_{D}\left(x, z, z^{\prime}\right) \leq c_{4}
$$

Proof of Lemma 4.5. Observe that $\xi_{D}\left(x, z, z^{\prime}\right)=\xi_{\mathcal{B}(0,1)}\left(0, \Psi(z), \Psi\left(z^{\prime}\right)\right)$ for $\Psi$ a conformal map from $D$ to $\mathcal{B}(0,1)$ which maps $x$ to 0 . It is well-known (see Lawler [10], Chapter 2, formula (2.6)) that for any $|a|<1$ and $|b|=1$,

$$
H_{\mathcal{B}(0,1)}(a, b)=\frac{1-|a|^{2}}{2 \pi|a-b|^{2}}
$$

It follows that for $\left|b^{\prime}\right|=|b|=1, H_{\mathcal{B}(0,1)}\left(b^{\prime}, b\right)=\frac{1}{\pi\left|b-b^{\prime}\right|^{2}}$ (see Lawler [10], Chapter 5, Example 5.6).

If $z \in \partial D$, then $|\Psi(z)|=1$ and $\xi_{\mathcal{B}(0,1)}\left(0, \Psi(z), \Psi\left(z^{\prime}\right)\right)=\frac{1}{2}\left|\Psi(z)-\Psi\left(z^{\prime}\right)\right|^{2} \leq 2$ yielding (4.6).

It remains to treat the case $z \in D$ with $|z-x|>\delta$. Write $a:=\Psi(z)$ for notational brevity. Using the elementary fact that for any $|a|<1, H_{\mathcal{B}(0,1)}(0, a)=\frac{1}{2 \pi} \log \frac{1}{|a|}$, we have

$$
\xi_{\mathcal{B}(0,1)}\left(0, \Psi(z), \Psi\left(z^{\prime}\right)\right)=\left(\log \frac{1}{|a|}\right) \frac{\left|a-\Psi\left(z^{\prime}\right)\right|^{2}}{1-|a|^{2}} \leq \frac{4 \log \frac{1}{|a|}}{1-|a|}
$$

where we used $\left|a-\Psi\left(z^{\prime}\right)\right| \leq 2$ by the triangular inequality and $1-|a|^{2} \geq 1-|a|$.

Now we estimate $|a|$ from below. Observe that $|z-x|=\left|\Psi^{-1}(a)-\Psi^{-1}(0)\right| \leq$ $|a| \sup _{0 \leq t \leq 1}\left|\left(\Psi^{-1}\right)^{\prime}(a t)\right|$. Applying Corollary 3.19 (Lawler [10], Chapter 3) to the conformal transformation $\Psi^{-1}$ which maps $\mathcal{B}(0,1)$ to $D$, we get that for any $0 \leq t \leq 1$, 
$\left|\left(\Psi^{-1}\right)^{\prime}(a t)\right| \leq 4 \frac{d\left(\Psi^{-1}(a t), \partial D\right)}{1-|a t|} \leq 4 \frac{\operatorname{diam}(D)}{1-|a|}$, where $\operatorname{diam}(D)$ denotes the diameter of $D$. Then $|z-x| \leq 4 \operatorname{diam}(D) \frac{|a|}{1-|a|}$. Since $|z-x|>\delta$ by assumption, we deduce that $|a| \geq \eta$, with $\eta:=\frac{\delta}{4 \operatorname{diam}(D)+\delta}$. Going back to $(4.9)$, we get that $\xi_{D}\left(x, z, z^{\prime}\right)=\xi_{\mathcal{B}(0,1)}\left(0, \Psi(z), \Psi\left(z^{\prime}\right)\right) \leq$ $\sup _{\eta \leq r<1} \frac{4 \log \frac{1}{r}}{1-r}=: c_{4}$, proving (4.7).

From (4.8), we have the following lemma.

Lemma 4.6 For each $\varepsilon>0$, there exists $\eta>0$ such that $\left|\frac{H_{\mathcal{B}(0,1)}(a, b)}{H_{\mathcal{B}(0,1)}(0, b)}-1\right|<\varepsilon$ for any $|b|=1$ and $|a|<\eta$.

In view of the proof of Proposition 4.2, we now study loops from $x$ to $x$ under $\mathbb{Q}_{x, D}^{z, z^{\prime}, \alpha}$. For any $r>0$, we denote by

$$
\begin{aligned}
N(x, r) & :=\#\{\text { loops from } x \text { to } x \text { which hit } \mathcal{C}(x, r)\} \\
\Upsilon(x, r) & :=\sum_{\mathfrak{e} \in \mathcal{E}_{\mathcal{B}(x, r)}} \xi_{\mathcal{B}(x, r)}\left(x, \mathfrak{e}_{g}, \mathfrak{e}_{d}\right) 1_{\{x \notin \mathfrak{e}\}},
\end{aligned}
$$

where $\mathcal{E}_{\mathcal{B}(x, r)}$ is the set of excursions inside $\mathcal{B}(x, r)$ as defined in Notation 3.3, and $\xi$ is defined in (3.1).

Let $z \neq z^{\prime}$ be distinct nice points of $\bar{D}$, different from $x$. Let $r_{0} \in(0,1)$ be such that $\mathcal{B}\left(x, r_{0}\right) \subset D$ and $z, z^{\prime} \notin \overline{\mathcal{B}\left(x, r_{0}\right)}$. Let $r_{k}:=\frac{r_{0}}{2^{k}}$ for $k \geq 0$.

Lemma 4.7 Let $\alpha \geq 0$. As $k \rightarrow \infty$,

(i) $N\left(x, r_{k}\right) \sim \alpha \log \frac{1}{r_{k}}, \quad \mathbb{Q}_{x, D}^{z, z^{\prime}, \alpha}$-a.s.;

(ii) $\Upsilon\left(x, r_{k}\right) \sim \alpha\left(\log \frac{1}{r_{k}}\right)^{2}, \quad \mathbb{Q}_{x, D}^{z, z^{\prime}, \alpha}$-a.s.

Proof of Lemma 4.7. (i) We start by mentioning a simple fact on the concentration of a Poisson variable: Let $N_{\lambda}$ be a Poisson random variable with parameter $\lambda>0$. By the standard large deviation principle, for any $\varepsilon>0$, there exist some $\delta>0$ and $\lambda_{0}>0$ such that for all $\lambda>\lambda_{0}$,

$$
\mathbb{P}\left(\left|N_{\lambda}-\lambda\right| \geq \varepsilon \lambda\right) \leq \mathrm{e}^{-\delta \lambda}
$$

Under $\mathbb{Q}_{x, D}^{z, z^{\prime}, \alpha}$, the number of loops from $x$ to $x$ that hit $\mathcal{C}\left(x, r_{k}\right)$ is a Poisson variable of parameter $\alpha \nu_{D}(x, x)\left(T_{\mathcal{C}\left(x, r_{k}\right)}<T_{x}\right)$ (see Section 1.2). By Lemma 2.3 (ii), $\nu_{D}(x, x)\left(T_{\mathcal{C}\left(x, r_{k}\right)}<\right.$ $\left.T_{x}\right)=\int_{\mathcal{C}\left(x, r_{k}\right)} G_{D}(x, y) H_{\mathcal{B}\left(x, r_{k}\right)}(x, y) \mathrm{d} y \sim \log \left(1 / r_{k}\right)$ as $k \rightarrow \infty$. By (4.12) and the BorelCantelli lemma, $N\left(x, r_{k}\right) \sim \alpha \log \left(1 / r_{k}\right)$ as $k \rightarrow \infty, \mathbb{Q}_{x, D}^{z, z^{\prime}, \alpha}$-a.s. This proves (i). 
(ii) For each $r>0$, the contribution to the right-hand side of (4.11) comes from the paths of the excursion from $z$ to $x$, of the $N(x, r)$ loops which hit $\mathcal{C}(x, r)$, and of the excursion from $x$ to $z^{\prime}$. Consequently for any $k \geq 1$,

$$
\Upsilon\left(x, r_{k}\right)=\Upsilon_{\text {begin }}+\sum_{j=1}^{N\left(x, r_{k}\right)} \Upsilon_{j}+\Upsilon_{e n d}
$$

where under $\mathbb{Q}_{x, D}^{z, z^{\prime}, \alpha}, \Upsilon_{\text {begin }}, \Upsilon_{\text {end }}, \Upsilon_{1}, \Upsilon_{2}, \ldots$, are mutually independent (and independent of $\left.N\left(x, r_{k}\right)\right)$ such that $\Upsilon_{\text {begin }}$ and $\Upsilon_{\text {end }}$ are distributed as $\Upsilon\left(x, r_{k}\right)$ under $\mathbb{P}_{D}^{z, x}$ and $\mathbb{P}_{D}^{x, z^{\prime}}$ respectively, and for any $j \geq 1, \Upsilon_{j}$ has the same distribution as that of $\Upsilon\left(x, r_{k}\right)$ under $\nu_{D}(x, x)\left(\bullet \mid T_{\mathcal{C}\left(x, r_{k}\right)}<T_{x}\right)$. [For the sake of presentation, we have introduced $\Upsilon_{j}$ for all $j \geq 1$; we have also omitted the dependence on $k$ in the notation $\Upsilon_{\text {begin }}, \Upsilon_{\text {end }}$ and $\Upsilon_{j}$, $j \geq 1$.]

We claim that

$$
\mathbb{Q}_{x, D}^{z, z^{\prime}, \alpha}\left(\Upsilon_{\text {begin }}\right)=\mathbb{Q}_{x, D}^{z, z^{\prime}, \alpha}\left(\Upsilon_{\text {end }}\right)=\mathbb{Q}_{x, D}^{z, z^{\prime}, \alpha}\left(\Upsilon_{1}\right)=C_{D, \mathcal{B}\left(x, r_{k}\right)}(x)
$$

In fact, notice that $H_{\mathcal{B}\left(x, r_{k}\right)}(x, z)=0$ as well as $H_{\mathcal{B}\left(x, r_{k}\right)}\left(x, z^{\prime}\right)=0$. Applying (3.17) to $L=1$ gives that $\mathbb{Q}_{x, D}^{z, z^{\prime}, \alpha}\left(\Upsilon_{\text {begin }}\right)=C_{D, \mathcal{B}\left(x, r_{k}\right)}(x)$. In the definition of $\Upsilon(x, r)$ in $(4.11)$, $\xi_{\mathcal{B}(x, r)}\left(x, \mathfrak{e}_{g}, \mathfrak{e}_{d}\right)=\xi_{\mathcal{B}(x, r)}\left(x, \mathfrak{e}_{d}, \mathfrak{e}_{g}\right)$, so by the time-reversal property (2.9) for the path from $x$ to $z^{\prime}$ and another application of $(3.17)$ to $L=1$, we get $\mathbb{Q}_{x, D}^{z, z^{\prime}, \alpha}\left(\Upsilon_{\text {end }}\right)=C_{D, \mathcal{B}\left(x, r_{k}\right)}(x)$. Finally, recall that $\nu_{D}(x, x)\left(T_{\mathcal{C}\left(x, r_{k}\right)}<T_{x}\right)=C_{D, \mathcal{B}\left(x, r_{k}\right)}(x)$ by definition (see Lemma 2.3). Applying (3.18) to $L=1$ gives that $\mathbb{Q}_{x, D}^{z, z^{\prime}, \alpha}\left(\Upsilon_{1}\right)=C_{D, \mathcal{B}\left(x, r_{k}\right)}(x)$ and completes the justification of (4.13).

By Lemma 2.3 (ii), $C_{D, \mathcal{B}\left(x, r_{k}\right)}(x)=\int_{\mathcal{C}\left(x, r_{k}\right)} G_{D}(x, y) H_{\mathcal{B}\left(x, r_{k}\right)}(x, y) \mathrm{d} y \sim \log \left(1 / r_{k}\right)$ as $k \rightarrow \infty$. [A fact already used in the proof of (i)].

From (i), the statement (ii) immediately follows once we have shown that for any deterministic sequence $n_{k}$ such that $\liminf _{k \rightarrow \infty} \frac{n_{k}}{k}>0$,

$$
\frac{1}{n_{k}^{2}}\left[\Upsilon_{\text {begin }}+\Upsilon_{\text {end }}+\sum_{j=1}^{n_{k}} \Upsilon_{j}-\left(n_{k}+2\right) C_{D, \mathcal{B}\left(x, r_{k}\right)}(x)\right] \rightarrow 0, \quad \mathbb{Q}_{x, D}^{z, z^{\prime}, \alpha} \text {-a.s. }
$$

To get (4.14), we shall use the following inequality (see Petrov [17], Theorem 2.10): There exists some constant $c_{1}>0$ such that for any sequence of independent real-valued integrable random variables $\left(\eta_{i}\right)_{i \geq 1}$,

$$
\mathbb{E}\left|\sum_{i=1}^{n}\left(\eta_{i}-\mathbb{E}\left(\eta_{i}\right)\right)\right|^{3} \leq c_{1} n^{1 / 2} \sum_{i=1}^{n} \mathbb{E}\left[\left|\eta_{i}\right|^{3}\right], \quad \forall n \geq 1
$$


Using the fact that $\xi_{\mathcal{B}\left(x, r_{k}\right)}\left(x, \mathfrak{e}_{g}, \mathfrak{e}_{d}\right)$ is bounded by 2 (see (4.6)), we deduce from the definition of $\Upsilon\left(x, r_{k}\right)$ in (4.11) that for some numerical constant $c_{2}$ [we may take $c_{2}=24$ ],

$$
\Upsilon\left(x, r_{k}\right)^{3} \leq c_{2} \sum_{L=1}^{3} \sum_{\underline{\underline{e}}_{\mathcal{B}^{L}\left(x, r_{k}\right)}^{L}} \prod_{j=1}^{L} \xi_{\mathcal{B}\left(x, r_{k}\right)}\left(x, \mathfrak{e}_{g}^{j}, \mathfrak{e}_{d}^{j}\right) 1_{\left\{\mathfrak{e}_{g}^{1} \neq x, \mathfrak{e}_{d}^{L} \neq x\right\}}
$$

where as in the previous section, $\sum_{\underline{\underline{e}}_{\mathcal{B}\left(x, r_{k}\right)}^{L}}$ is a short way to denote sum over all ordered (distinct) excursions $\left(\mathfrak{e}^{1}, \ldots, \mathfrak{e}^{L}\right) \in\left(\mathcal{E}_{\mathcal{B}\left(x, r_{k}\right)}\right)^{L}$.

From the equations (3.17) and (3.18) with $L \in\{1,2,3\}, D_{1}=\mathcal{B}\left(x, r_{k}\right)$ there, we see that there exists some positive constant $c_{3}=c_{3}(x)$ such that the third moments of $\Upsilon_{\text {begin }}$, of $\Upsilon_{\text {end }}$ and of $\Upsilon_{1}$ are less than $c_{3}\left(\log \left(1 / r_{k}\right)\right)^{3}$.

Recalling (4.13). It follows from (4.15) that

$$
\begin{aligned}
& \mathbb{Q}_{x, D}^{z, z^{\prime}, \alpha}\left[\left|\frac{1}{n_{k}^{2}}\left(\Upsilon_{\text {begin }}+\Upsilon_{\text {end }}+\sum_{j=1}^{n_{k}} \Upsilon_{j}-\left(n_{k}+2\right) C_{D, \mathcal{B}\left(x, r_{k}\right)}(x)\right)\right|^{3}\right] \\
\leq & c_{1} c_{3} \frac{\left(n_{k}+2\right)^{3 / 2}}{n_{k}^{6}}\left(\log \frac{1}{r_{k}}\right)^{3},
\end{aligned}
$$

which is summable in $k$ thanks to the assumption on $n_{k}: \liminf \inf _{k \rightarrow \infty} \frac{n_{k}}{k}>0$. The BorelCantelli lemma yields (4.14) and completes the proof of (ii).

\subsection{Proof of Proposition 4.2}

We fix $D, z \neq z^{\prime}$ nice points of $\bar{D}$. Let $0 \leq \alpha<2$ and $0 \leq \varrho<2-\alpha$. Take $\gamma=\gamma(\varrho)$ and $\varepsilon=\varepsilon(\varrho)$ such that $\gamma>\alpha, \varepsilon>0$ and

$$
2(1+\varepsilon) \sqrt{\gamma \alpha}-\alpha+\varrho<2
$$

Let $\eta \in(0,1)$ be the constant in Lemma 4.6 associated with our choice of $\varepsilon$. Let $K \geq 5$ be such that $2^{-K}<\frac{\eta}{16}$. Constants $c_{5}, c_{6}, \ldots$ in the proof can depend on $D, z, z^{\prime}, \alpha, \varrho, \gamma$, $\varepsilon, K$ even if not specified.

Let $x \in D$ be a suitable point, meaning, as before, that $x$ is in some square $D_{n} \in \mathscr{D}_{n}$ at any level $n$, and is different from $z$ and $z^{\prime}$ (recall that the set of suitable points has full Lebesgue measure). Let $r_{0} \in(0,1)$ and $r_{k}=\frac{r_{0}}{2^{k}}$, let

$$
\begin{aligned}
E^{(x)}\left(r_{0}, \gamma\right):= & \left\{d\left(x, \partial D \cup\left\{z, z^{\prime}\right\}\right)>r_{0}\right\} \cap \\
& \cap_{k \geq 0}\left\{N\left(x, r_{k}\right) \leq \gamma \log \frac{1}{r_{k}}, \Upsilon\left(x, r_{k}\right) \leq \gamma\left(\log \frac{1}{r_{k}}\right)^{2}\right\} .
\end{aligned}
$$


We assume, for the moment, that for all suitable $x \in D$,

$$
\limsup _{n \rightarrow+\infty} 1_{\left\{d\left(x, \partial D_{n}^{(x)}\right) \geq \frac{2^{-n}}{4}\right\}} \mathbb{E}_{\mathbb{Q}_{x, D}^{z, z^{\prime}, \alpha}}\left[\int_{D} \frac{\mathcal{M}_{\mathscr{D}_{n}}^{\alpha}(\mathrm{d} u)}{|u-x|^{\varrho}}, E^{(x)}\left(r_{0}, \gamma\right)\right] \leq c_{5}\left(r_{0}\right),
$$

where $c_{5}\left(r_{0}\right)$ is some positive constant depending on $r_{0}$. We claim that Proposition 4.2 will follow from Lemma 4.7 and (the case $\varrho=0$ of) (4.18). In fact, for any $c>0$, we deduce from (4.18) (with $\varrho=0$ ) that

$$
\begin{aligned}
& \liminf _{n \rightarrow+\infty} \mathbb{E}_{\mathbb{Q}_{x, D}^{z, z^{\prime}, \alpha}}\left[\min \left(c, \mathcal{M}_{\mathscr{D}_{n}}^{\alpha}(D)\right), E^{(x)}\left(r_{0}, \gamma\right)\right] \\
\leq & c_{5}\left(r_{0}\right)+c \liminf _{n \rightarrow+\infty} 1_{\left\{d\left(x, \partial D_{n}^{(x)}\right)<\frac{r_{n}}{4}\right\}} \\
= & c_{5}\left(r_{0}\right),
\end{aligned}
$$

for Lebesgue-a.e. $x \in D$. By Fatou's lemma, it gives that for any $c>0$,

$$
\mathbb{E}_{\mathbb{Q}_{x, D}^{z, z^{\prime}, \alpha}}\left[\min \left(c, \mathcal{M}_{\infty}^{\alpha}(D)\right), E^{(x)}\left(r_{0}, \gamma\right)\right] \leq c_{5}\left(r_{0}\right)
$$

then, by monotone convergence,

$$
\mathbb{E}_{\mathbb{Q}_{x, D}^{z, z^{\prime}, \alpha}}\left[\mathcal{M}_{\infty}^{\alpha}(D), E^{(x)}\left(r_{0}, \gamma\right)\right] \leq c_{5}\left(r_{0}\right)
$$

We deduce that for Lebesgue-a.e. $x \in D, \mathbb{Q}_{x, D}^{z, z^{\prime}, \alpha}$-a.s., $\mathcal{M}_{\infty}^{\alpha}(D)<\infty$ on the event $E^{(x)}\left(r_{0}, \gamma\right)$. By Lemma 4.7, we have for any $x \in D \backslash\left\{z, z^{\prime}\right\}$,

$$
\mathbb{Q}_{x, D}^{z, z^{\prime}, \alpha}\left(\bigcup_{\ell=1}^{\infty} E^{(x)}\left(2^{-\ell}, \gamma\right)\right)=1 .
$$

Hence we get (4.4). [The case $0<\varrho<2-\alpha$ in (4.18) will be used in Section 6.]

It remains to prove (4.18). We fix $r_{0}>0$ and suitable $x \in D$ such that $d(x, \partial D \cup$ $\left.\left\{z, z^{\prime}\right\}\right)>r_{0}$. We write $\mathcal{B}_{0}:=D$ and $\mathcal{B}_{k}:=\mathcal{B}\left(x, r_{k}\right)$ for any $k \geq 1$. We distinguish three possible situations: (i) $|u-x|>r_{K}$ with $u \notin D_{n}^{(x)}$, (ii) $|u-x|<r_{K}$ with $u \notin D_{n}^{(x)}$, and (iii) $u \in D_{n}^{(x)}$. We suppose that $d\left(x, \partial D_{n}^{(x)}\right) \geq \frac{2^{-n}}{4}$, which means that $x$ is not too close to the boundary of $D_{n}^{(x)}$.

First case: $|u-x|>r_{K}$ with $u \notin D_{n}^{(x)} \quad$ Since we are going to integrate over $u$ with respect to the Lebesgue measure, we suppose, without loss of generality, that $u$ is a suitable point. With the notation of Proposition 3.9, observe that $\widetilde{M}_{x, D_{n}^{(u)}}(u, \alpha)=M_{D_{n}^{(u)}}(u, \alpha)$ since the excursions inside $D_{n}^{(u)}$ cannot hit $x$. Recall the definition of $\Upsilon_{x, D_{1}, S, D_{2}}(u)$ in (3.24). We are going to take $D_{1}=D, S=\mathcal{B}_{K}$ and $D_{2}=D_{n}^{(u)}$. Then $\Upsilon_{x, D, \mathcal{B}_{K}, D_{n}^{(u)}}(u)$ is 
(possibly) contributed by excursions from $z$ to $x$, from $x$ to $z^{\prime}$ and by the loops at $x$ in $D$ which hit $\mathcal{C}\left(x, r_{K}\right)$. By definition,

$$
\begin{aligned}
& \Upsilon_{x, D, \mathcal{B}_{K}, D_{n}^{(u)}}(u) \\
= & \frac{2 \pi}{H_{D}(z, x)}\left(H_{D}(u, z)-H_{D_{n}^{(u)}}(u, z)\right)\left(H_{D}(u, x)-H_{D_{n}^{(u)}}(u, x)\right) \\
& +\frac{2 \pi}{H_{D}\left(x, z^{\prime}\right)}\left(H_{D}\left(u, z^{\prime}\right)-H_{D_{n}^{(u)}}\left(u, z^{\prime}\right)\right)\left(H_{D}(u, x)-H_{D_{n}^{(u)}}(u, x)\right) \\
& +\frac{(2 \pi)^{2}}{C_{D, \mathcal{B}_{K}}(x)}\left(H_{D}(u, x)-H_{D_{n}^{(u)}}(u, x)\right)^{2} N\left(x, r_{K}\right),
\end{aligned}
$$

by recalling that $N\left(x, r_{K}\right)$ denotes the number of loops at $x$ which hit $\mathcal{C}\left(x, r_{K}\right)$. This implies that

$$
\Upsilon_{x, D, \mathcal{B}_{K}, D_{n}^{(u)}}(u) \leq \xi_{D}(u, x, z)+\xi_{D}\left(u, x, z^{\prime}\right)+\frac{G_{D}(u, x)^{2}}{C_{D, \mathcal{B}_{K}}(x)} N\left(x, r_{K}\right)=: c_{(4.21)},
$$

recalling that $G_{D}(u, x)=2 \pi H_{D}(u, x)$.

By applying Proposition 3.9 to $D_{1}=D, S=\mathcal{B}_{K}$ and $D_{2}=D_{n}^{(u)}$, we get that

$$
\begin{aligned}
\mathbb{E}_{\mathbb{Q}_{x, D}^{z, z^{\prime}, \alpha}}\left[M_{D_{n}^{(u)}}(u, \alpha) \mid N\left(x, r_{K}\right)\right] & =\mathbb{E}_{\mathbb{Q}_{x, D}^{z, z^{\prime}, \alpha}}\left[\widetilde{M}_{x, D_{n}^{(u)}}(u, \alpha) \mid N\left(x, r_{K}\right)\right] \\
& \leq c_{(4.21)} \mathrm{e}^{-\alpha C_{D}(u)} \mathrm{e}^{2 \sqrt{\alpha c(4.21)}} .
\end{aligned}
$$

Let us control the three terms in $c_{(4.21)}$. For the last term in $c_{(4.21)}$, we remark that since $|u-x|>r_{K}$, we have, by Lawler [10] Proposition 2.36, $G_{D}(u, x) \leq \log \operatorname{diam}(D)+$ $\log \frac{1}{r_{K}}$ (where $\operatorname{diam}(D)$ denotes, as before, the diameter of $\left.D\right)$. For any $|y-x|=r_{K}$, $G_{D}(x, y) \geq \log d(x, \partial D)+\log \frac{1}{r_{K}}$ (again by Lawler [10] Proposition 2.36). It follows that $C_{D, \mathcal{B}_{K}}(x)=\int_{\mathcal{C}\left(x, r_{K}\right)} G_{D}(x, y) H_{\mathcal{B}\left(x, r_{K}\right)}(x, y) \mathrm{d} y \geq \log \left(r_{0} / r_{K}\right)$. On the event $E^{(x)}\left(r_{0}, \gamma\right)$, we have $N\left(x, r_{K}\right) \leq \gamma\left(\log r_{K}\right)^{2}$, hence for some constant $c_{6}=c_{6}\left(r_{0}, K, D, \gamma\right)$,

$$
\frac{G_{D}(u, x)^{2}}{C_{D, \mathcal{B}_{K}}(x)} N\left(x, r_{K}\right) \leq c_{6} .
$$

To control $\xi_{D}(u, x, z)+\xi_{D}\left(u, x, z^{\prime}\right)$ in $c_{(4.21)}$, we discuss separately two cases: If $z \in \partial D$, then we apply (4.7) to see that $\xi_{D}(u, x, z) \leq c_{4}$. If $z \in D, H_{D}(u, x)$ is bounded as seen in the previous paragraph (recalling that $\left.|u-x|>r_{K}\right), H_{D}(x, z) \geq$ $\inf _{y: d(y, \partial D \cup\{z\}) \geq r_{0}} H_{D}(y, z)=: c_{7}\left(r_{0}, z, D\right)>0$, hence $\xi_{D}(u, x, z)=2 \pi \frac{H_{D}(u, x)}{H_{D}(x, z)} H_{D}(u, z) \leq$ $2 \pi c_{7} H_{D}(u, z)$. We get that for $z \in \bar{D}, \xi_{D}(u, x, z) \leq c_{4} 1_{\{z \in \partial D\}}+2 \pi c_{7} H_{D}(u, z) 1_{\{z \in D\}}=$ : $f_{z}(u)$. A similar bound holds for $\xi_{D}\left(u, x, z^{\prime}\right)$. Therefore we have shown that on the event $E^{(x)}\left(r_{0}, \gamma\right)$

$$
c_{(4.21)} \leq c_{6}+f_{z}(u)+f_{z^{\prime}}(u)=: f_{z, z^{\prime}}(u)
$$


Going back to (4.22), we see that

$$
\begin{aligned}
& \mathbb{E}_{\mathbb{Q}_{x, D}^{z, z^{\prime}, \alpha}}\left[M_{D_{n}^{(u)}}(u, \alpha), E^{(x)}\left(r_{0}, \gamma\right)\right] \\
\leq & \mathbb{E}_{\mathbb{Q}_{x, D}^{z, z^{\prime}, \alpha}}\left[M_{D_{n}^{(u)}}(u, \alpha), N\left(x, r_{K}\right) \leq \gamma\left(\log r_{K}\right)^{2}\right] \\
\leq & f_{z, z^{\prime}}(u) \mathrm{e}^{-\alpha C_{D}(u)} \mathrm{e}^{2 \sqrt{\alpha f_{z, z^{\prime}}(u)}},
\end{aligned}
$$

which implies that

$$
\begin{aligned}
& \mathbb{E}_{\mathbb{Q}_{x, D}^{z, z^{\prime}, \alpha}}\left[\int_{|u-x|>r_{K}} \frac{\mathcal{M}_{\mathscr{D}_{n}}^{\alpha}(\mathrm{d} u)}{|u-x|^{\varrho}}, E^{(x)}\left(r_{0}, \gamma\right)\right] \\
\leq & \int_{|u-x|>r_{K}} \frac{\mathrm{d} u}{|u-x|^{\varrho}} f_{z, z^{\prime}}(u) \mathrm{e}^{-\alpha C_{D}(u)} \mathrm{e}^{2 \sqrt{\alpha f_{z, z^{\prime}}(u)}} \\
\leq & c_{9},
\end{aligned}
$$

with $c_{9}:=r_{K}^{-\varrho} \int_{D} f_{z, z^{\prime}}(u) \mathrm{e}^{-\alpha C_{D}(u)} \mathrm{e}^{2 \sqrt{\alpha f_{z, z^{\prime}}(u)}} \mathrm{d} u<\infty$.

Second case: $r_{k+K+1} \leq|u-x|<r_{k+K}$ for some $k \geq 0$, with $u \notin D_{n}^{(x)} \quad$ We still have $\widetilde{M}_{x, D_{n}^{(u)}}(u, \alpha)=M_{D_{n}^{(u)}}(u, \alpha)$. We claim

$$
\overline{D_{n}^{(u)}} \subset \mathcal{B}_{k}, \quad \partial D_{n}^{(u)} \subset \mathcal{B}_{k+K+5}^{c} .
$$

To see why $\overline{D_{n}^{(u)}} \subset \mathcal{B}_{k}$ holds: It suffices to check $|u-x|+\sqrt{2} \times 2^{-n}<r_{k}$, which is easy. Indeed, $|u-x|<r_{k+K}<\frac{1}{2} r_{k}$ (since $K>1$ ), and since $d\left(x, \partial D_{n}^{(x)}\right) \geq \frac{1}{4} 2^{-n}$, we also have $\sqrt{2} \times 2^{-n}<\sqrt{2} \times 4 d\left(x, \partial D_{n}^{(x)}\right) \leq \sqrt{2} \times 4|u-x|<\sqrt{2} \times 4 r_{k+K}$ which is smaller than $\frac{1}{2} r_{k}$ (since $\left.K \geq 4\right)$.

We now prove the second inclusion in (4.24) by discussing on two possible situations. If $r_{k+K+1} \geq \frac{4}{2^{n}}$, then trivially $|u-x| \geq r_{k+K+1}>r_{k+K+5}+\sqrt{2} \times 2^{-n}$, which yields $\partial D_{n}^{(u)} \subset \mathcal{B}_{k+K+5}^{c}$. If, on the other hand, $r_{k+K+1}<\frac{4}{2^{n}}$, then $r_{k+K+5}<\frac{1}{4} 2^{-n}$, which yields $\mathcal{B}_{k+K+5} \subset\left(\partial D_{n}^{(u)}\right)^{c}$ (because $\left.d\left(x, \partial D_{n}^{(x)}\right) \geq \frac{1}{4} 2^{-n}\right)$, which, in turn, implies $\partial D_{n}^{(u)} \subset$ $\mathcal{B}_{k+K+5}^{c}$. As such, (4.24) is proved.

Recall the definition of $\Upsilon_{x, D_{1}, S, D_{2}}(u)$ in (3.24); we take $D_{1}=\mathcal{B}_{k}, S=\mathcal{B}_{k+K+5}$ and $D_{2}=D_{n}^{(u)}$. Then $\Upsilon_{x, \mathcal{B}_{k}, \mathcal{B}_{k+K+5}, D_{n}^{(u)}}(u)$ is (possibly) contributed by excursions from $\mathcal{C}\left(x, r_{k}\right)$ to $x$, from $x$ to $\mathcal{C}\left(x, r_{k}\right)$, by the loops in $\mathcal{B}_{k}$ which hit $\mathcal{C}\left(x, r_{k+K+5}\right)$, and by excursions from $\mathcal{C}\left(x, r_{k}\right)$ to itself without hitting $x$. [The latter excursions make the most significant contribution to $\Upsilon_{x, \mathcal{B}_{k}, \mathcal{B}_{k+K+5}, D_{n}^{(u)}}(u)$.] We claim that for some positive constant $c_{10}$ only depending on $K$ and all $\mathfrak{e} \in \mathcal{E}_{x, \mathcal{B}_{k}, \mathcal{B}_{k+K+5}}$,

$$
\xi_{x, \mathcal{B}_{k}, \mathcal{B}_{k+K+5}, D_{n}^{(u)}}\left(u, \mathfrak{e}_{g}, \mathfrak{e}_{d}\right) \leq c_{10}
$$


To prove $(4.25)$, we start by noting that $H_{D_{n}^{(u)}}(u, x)=0$ (because $x \notin \overline{D_{n}^{(u)}}$ ) and $H_{D_{n}^{(u)}}(u, y)=0$ for any $y \in \partial \mathcal{B}_{k}=\mathcal{C}\left(x, r_{k}\right)$. Hence by $(3.22)$,

$$
\begin{aligned}
\xi_{x, \mathcal{B}_{k}, \mathcal{B}_{k+K+5}, D_{n}^{(u)}}\left(u, \mathfrak{e}_{g}, \mathfrak{e}_{d}\right)= \\
\begin{cases}(2 \pi)^{2}\left(H_{\mathcal{B}_{k}}(u, x)\right)^{2} / C_{\mathcal{B}_{k}, \mathcal{B}_{k+K+5}}(x), & \text { if } \mathfrak{e}_{g}=\mathfrak{e}_{d}=x, \\
\xi_{\mathcal{B}_{k}}\left(u, \mathfrak{e}_{g}, \mathfrak{e}_{d}\right), & \text { otherwise. }\end{cases}
\end{aligned}
$$

If $\mathfrak{e}_{g}, \mathfrak{e}_{d} \in \mathcal{C}\left(x, r_{k}\right)$, we use (4.7) to see that $\xi_{x, \mathcal{B}_{k}, \mathcal{B}_{k+K+5}, D_{n}^{(u)}}\left(u, \mathfrak{e}_{g}, \mathfrak{e}_{d}\right) \leq 2$; otherwise, we use the following explicit computations: $H_{\mathcal{B}_{k}}(u, x)=\frac{1}{2 \pi} \log \frac{r_{k}}{|u-x|}, C_{\mathcal{B}_{k}, \mathcal{B}_{k+K+5}}(x)=$ $\log \frac{r_{k}}{r_{k+K+5}}=(K+5) \log 2, H_{\mathcal{B}_{k}}(u, y)=\frac{1}{2 \pi r_{k}} \frac{r_{k}^{2}-|u-x|^{2}}{|u-y|^{2}}$ and $H_{\mathcal{B}_{k}}(x, y)=\frac{1}{2 \pi r_{k}}$ for any $y \in$ $\mathcal{C}\left(x, r_{k}\right)$. Since $r_{k+K+1} \leq|u-x|<r_{k+K}$, we easily get (4.25).

For $\mathfrak{e}$ inside $\mathcal{C}\left(x, r_{k}\right)$ which does not hit $x$, we have by our choice of $K$ and Lemma 4.6, $\xi_{\mathcal{B}_{k}}\left(u, \mathfrak{e}_{g}, \mathfrak{e}_{d}\right) \leq(1+\varepsilon)^{2} \xi_{\mathcal{B}_{k}}\left(x, \mathfrak{e}_{g}, \mathfrak{e}_{d}\right)$.

With the notation of (4.10), the number of excursions from $\mathcal{C}\left(x, r_{k}\right)$ to $x$, from $x$ to $\mathcal{C}\left(x, r_{k}\right)$, and the loops in $\mathcal{B}_{k}$ which hit $\mathcal{C}\left(x, r_{k+K+5}\right)$, is less than $2 N\left(x, r_{k+K+5}\right)+2$. [The presence of +2 is due to the path from $z$ to $x$, and to the path from $x$ to $z^{\prime}$.] In the notation of (4.11), we have

$$
\Upsilon_{x, D, \mathcal{B}(x, r), D_{n}^{(u)}}(u) \leq(1+\varepsilon)^{2} \Upsilon\left(x, r_{k}\right)+2 c_{10}\left(N\left(x, r_{k+K+5}\right)+1\right)=: c_{(4.26)}
$$

In view of (4.24), we are entitled to apply Proposition 3.9 to $D_{1}=\mathcal{B}_{k}, S=\mathcal{B}_{k+K+5}$ and $D_{2}=D_{n}^{(u)}$, to see that,

$$
\begin{aligned}
& \mathbb{E}_{\mathbb{Q}_{x, D}^{z, z^{\prime}, \alpha}}\left[M_{D_{n}^{(u)}}(u, \alpha) \mid N\left(x, r_{k+K+5}\right), \Upsilon\left(x, r_{k}\right)\right] \\
= & \mathbb{E}_{\mathbb{Q}_{x, D}^{z, z^{\prime}, \alpha}}\left[\widetilde{M}_{x, D_{n}^{(u)}}(u, \alpha) \mid N\left(x, r_{k+K+5}\right), \Upsilon\left(x, r_{k}\right)\right] \\
\leq & c_{(4.26)} \mathrm{e}^{-\alpha C_{\mathcal{B}_{k}}(u)} \mathrm{e}^{2 \sqrt{\alpha c(4.26)}}
\end{aligned}
$$

Recall the definition of $C_{S}(u)$ in Lemma 2.3. We see that $C_{\mathcal{B}_{k}}(u) \geq \log \left(1 /\left(2 r_{k}\right)\right)$. By the choice of the event $E^{(x)}\left(r_{0}, \gamma\right)$, we deduce the existence of a constant $c_{11}$ depending on $(K, \varepsilon, \alpha, \gamma)$ such that uniformly in $u$ satisfying $|u-x|<r_{K}$ and $u \notin D_{n}^{(x)}$,

$$
\mathbb{E}_{\mathbb{Q}_{x, D}^{z, z^{\prime}, \alpha}}\left[M_{D_{n}^{(u)}}(u, \alpha), E^{(x)}\left(r_{0}, \gamma\right)\right] \leq \frac{c_{11}}{|u-x|^{2(1+\varepsilon) \sqrt{\alpha \gamma}-\alpha}}\left(\log \frac{1}{|u-x|}\right)^{2} .
$$

Third (and last) case: $u \in D_{n}^{(x)}$ Here we assume that $n$ is large enough so that $D_{n}^{(x)} \subset \mathcal{B}\left(x, r_{0}\right)$. Recall that $d\left(x, \partial D_{n}^{(x)}\right) \geq \frac{2^{-n}}{4}$. Let $\mathfrak{a}_{n}$ be the smallest integer $j \geq K$ such that $r_{j}<\frac{2^{-n}}{4}$. In particular, $\mathcal{B}_{\mathfrak{a}_{n}} \subset D_{n}^{(x)}$, so the number of excursions in $\mathcal{E}_{D_{n}^{(x)}}$ 
which hit $x$ is smaller than $N:=N\left(x, r_{\mathfrak{a}_{n}}\right)+1$. For all $0 \leq m \leq L$, and for all $\widetilde{\mathfrak{e}}^{1}, \ldots, \widetilde{\mathfrak{e}}^{m}$ distinct and ordered excursions in $\mathcal{E}_{D_{n}^{(x)}}$ which do not hit $x$, consider the set of $\mathfrak{e}^{1}, \ldots, \mathfrak{e}^{L}$ distinct and ordered excursions in $\mathcal{E}_{D_{n}^{(x)}}$ such that $\left\{\widetilde{\mathfrak{e}}^{1}, \ldots, \widetilde{\mathfrak{e}}^{m}\right\} \subset\left\{\mathfrak{e}^{1}, \ldots, \mathfrak{e}^{L}\right\}$ and any $\mathfrak{e} \in$ $\left\{\mathfrak{e}^{1}, \ldots, \mathfrak{e}^{L}\right\} \backslash\left\{\widetilde{\mathfrak{e}}^{1}, \ldots, \widetilde{\mathfrak{e}}^{m}\right\}$ hits $x$. We remark that the cardinality of this set is less than $\left(\begin{array}{c}N \\ L-m\end{array}\right)$.

Recall from (4.6) that $\xi_{D_{n}^{(x)}}\left(u, y, y^{\prime}\right) \leq 2$ for any $y, y^{\prime} \in \partial D_{n}^{(x)}$. It follows that

$$
\sum_{\underline{\mathfrak{e}}_{D_{n}^{(x)}}^{L}} \prod_{j=1}^{L} \xi_{D_{n}^{(x)}}\left(u, \mathfrak{e}_{g}^{j}, \mathfrak{e}_{d}^{j}\right) \leq \sum_{m=0}^{L} 2^{L-m}\left(\begin{array}{c}
N \\
L-m
\end{array}\right) \Theta_{n}^{(m)}(u),
$$

where $\Theta_{n}^{(0)}(u):=1$ and for any $m \geq 1^{14}$

$$
\Theta_{n}^{(m)}(u):=\sum_{\underline{\mathfrak{e}}_{D_{n}^{(x)}}^{m}} \prod_{j=1}^{m} \xi_{D_{n}^{(x)}}\left(u, \mathfrak{e}_{g}^{j}, \mathfrak{e}_{d}^{j}\right) 1_{\left\{x \notin \mathfrak{e}^{j}\right\}} .
$$

We get that for any $u \in D_{n}^{(x)}$,

$$
\begin{aligned}
& M_{D_{n}^{(x)}}(u, \alpha) \\
\leq & \mathrm{e}^{-\alpha C_{D_{n}^{(x)}}(u)} \sum_{L=1}^{\infty} \frac{\alpha^{L-1}}{(L-1) !} \sum_{m=0}^{L} 2^{L-m}\left(\begin{array}{c}
N \\
L-m
\end{array}\right) \Theta_{n}^{(m)}(u) \\
\leq & \mathrm{e}^{-\alpha C_{D_{n}^{(x)}}^{(u)}} \sum_{m=0}^{\infty} \Theta_{n}^{(m)}(u) \sum_{L=\max (m, 1)}^{\infty} 2^{L-m} \frac{\alpha^{L-1}}{(L-1) !}\left(\begin{array}{c}
N \\
L-m
\end{array}\right) .
\end{aligned}
$$

To estimate the $\operatorname{sum} \sum_{L=\max (m, 1)}^{\infty}(\cdots)$ in (4.28), we shall use the following elementary inequalities: for any $b$ and $j$ two integers and for all $s \geq 0$,

$$
\sum_{\ell=0}^{\infty} \frac{s^{\ell}}{(b+\ell) !}\left(\begin{array}{l}
j \\
\ell
\end{array}\right) \leq \frac{1}{b !} \sum_{\ell=0}^{\infty} \frac{s^{\ell}}{\ell !}\left(\begin{array}{l}
j \\
\ell
\end{array}\right) \leq \frac{1}{b !} \sum_{\ell=0}^{\infty} \frac{(s j)^{\ell}}{(\ell !)^{2}} \leq \frac{1}{b !} \mathrm{e}^{2 \sqrt{s j}} .
$$

When $m=0$, the $\operatorname{sum} \sum_{L=\max (m, 1)}^{\infty}(\cdots)$ in (4.28) is equal to

$$
\begin{aligned}
\sum_{L=1}^{\infty} 2^{L} \frac{\alpha^{L-1}}{(L-1) !}\left(\begin{array}{c}
N \\
L
\end{array}\right) & =2 \sum_{\ell=0}^{\infty} \frac{(2 \alpha)^{\ell}}{\ell !}\left(\begin{array}{c}
N \\
\ell+1
\end{array}\right) \\
& \leq 2 N \sum_{\ell=0}^{\infty} \frac{(2 \alpha)^{\ell}}{\ell !}\left(\begin{array}{c}
N-1 \\
\ell
\end{array}\right) \\
& \leq 2 N \mathrm{e}^{2 \sqrt{2 \alpha(N-1)}},
\end{aligned}
$$

\footnotetext{
${ }^{14}\left(\begin{array}{c}N \\ L-m\end{array}\right)=0$ if $N<L-m$.
} 
by using (4.29) for the last inequality. When $m \geq 1$, the $\operatorname{sum} \sum_{L=\max (m, 1)}^{\infty}(\cdots)$ in $(4.28)$ is equal to, after a change of variables $L=m+\ell$,

$$
\alpha^{m-1} \sum_{\ell=0}^{\infty} \frac{(2 \alpha)^{\ell}}{(m-1+\ell) !}\left(\begin{array}{c}
N \\
\ell
\end{array}\right) \leq \frac{\alpha^{m-1}}{(m-1) !} \mathrm{e}^{2 \sqrt{2 \alpha N}},
$$

by using again (4.29) for the last inequality. It follows from (4.28) that

$$
\begin{aligned}
M_{D_{n}^{(x)}}(u, \alpha) & \leq \mathrm{e}^{-\alpha C_{D_{n}^{(x)}}(u)}\left[2 N+\sum_{m=1}^{\infty} \frac{\alpha^{m-1}}{(m-1) !} \Theta_{n}^{(m)}(u)\right] \mathrm{e}^{2 \sqrt{2 \alpha N}} \\
& =\left[2 N \mathrm{e}^{-\alpha C_{D_{n}^{(x)}}^{(u)}}+\widetilde{M}_{x, D_{n}^{(x)}}(u, \alpha)\right] \mathrm{e}^{2 \sqrt{2 \alpha N}}
\end{aligned}
$$

with the notation of Proposition 3.9. We want to use Proposition 3.9 with $D_{1}=\mathcal{B}_{\mathfrak{a}_{n}-K}$, $D_{2}=D_{n}^{(x)}$ and $S=\mathcal{B}_{\mathfrak{a}_{n}}$ (Note that $D_{n}^{(x)} \subset \mathcal{B}_{\mathfrak{a}_{n}-K}$ as $K \geq 4$ ). Remark that uniformly in $u \in D_{n}^{(x)}$,

$$
0 \leq H_{\mathcal{B}_{\mathfrak{a}_{n}-K}}(u, x)-H_{D_{n}^{(x)}}(u, x) \leq c_{12}(K)
$$

In fact, if $|u-x| \geq \frac{2^{-n}}{8}$, then $H_{\mathcal{B}_{\mathfrak{a}_{n}-K}}(u, x)=\frac{1}{2 \pi} \log \frac{r_{\mathfrak{a}_{n}-K}}{|u-x|} \leq \frac{\log 2}{2 \pi}(K+1)$, by using the fact that $r_{\mathfrak{a}_{n}}<\frac{2^{-n}}{4}$. Now if $|u-x|<\frac{2^{-n}}{8}$, then $d\left(u, \partial D_{n}^{(x)}\right)>\frac{2^{-n}}{8}$ (recall that $d\left(x, \partial D_{n}^{(x)}\right)>$ $\left.\frac{2^{-n}}{4}\right)$. By Lawler [10], Proposition 2.36, $H_{\mathcal{B}_{\mathfrak{a}_{n}-K}}(u, x)-H_{D_{n}^{(x)}}(u, x)=\frac{1}{2 \pi} \mathbb{E}^{x} \log \mid B_{T_{\partial \mathcal{B}_{\mathfrak{a}_{n}-K}}}-$ $u\left|-\frac{1}{2 \pi} \mathbb{E}^{x} \log \right| B_{T_{\partial D_{n}^{(x)}}}-u \mid \leq \frac{1}{2 \pi} \log \frac{2 r_{\mathfrak{a}_{n}-K}}{\left(2^{-n} / 8\right)} \leq \frac{\log 2}{2 \pi}(K+2)=: c_{12}(K)$. This proves (4.31).

Using (4.31), the similar computations leading to (4.25) show that there is some positive constant $c_{13}$ only depending on $K$ such that for all $u \in D_{n}^{(x)}$, for all $\mathfrak{e} \in \mathcal{E}_{x, \mathcal{B}_{\mathfrak{a}_{n}-K}, \mathcal{B}_{\mathfrak{a}_{n}}}$,

$$
\xi_{x, \mathcal{B}_{\mathfrak{a}_{n}-K}, \mathcal{B}_{\mathfrak{a}_{n}, D_{n}}^{(x)}}\left(u, \mathfrak{e}_{g}, \mathfrak{e}_{d}\right) \leq c_{13} .
$$

Recall the definition of $\Upsilon_{x, D_{1}, S, D_{2}}(u)$ in (3.24); we take $D_{1}=\mathcal{B}_{\mathfrak{a}_{n}-K}, D_{2}=D_{n}^{(x)}$ and $S=\mathcal{B}_{\mathfrak{a}_{n}}$. Remark that for all $u \in D_{n}^{(x)},|u-x|<\sqrt{2} 2^{-n}<\sqrt{2} r_{\mathfrak{a}_{n}-1}<\eta r_{\mathfrak{a}_{n}-K}$ by the choice of $K$. By Lemma 4.6, for all $y \in \mathcal{C}\left(x, r_{\mathfrak{a}_{n}-K}\right), H_{\mathcal{B}_{\mathfrak{a}_{n}-K}}(u, y) \leq(1+\varepsilon) H_{\mathcal{B}_{\mathfrak{a}_{n}-K}}(x, y)$. Then similarly to (4.26), we have that

$$
\Upsilon_{x, \mathcal{B}_{\mathfrak{a}_{n}-K}, \mathcal{B}_{\mathfrak{a}_{n}}, D_{n}^{(x)}}(u) \leq(1+\varepsilon)^{2} \Upsilon\left(x, r_{\mathfrak{a}_{n}-K}\right)+2 c_{13}\left(N\left(x, r_{\mathfrak{a}_{n}}\right)+1\right)=: c_{(4.32)}
$$

Applying Proposition 3.9 to $D_{1}=\mathcal{B}_{\mathfrak{a}_{n}-K}, D_{2}=D_{n}^{(x)}$ and $S=\mathcal{B}_{\mathfrak{a}_{n}}$ gives that

$$
\begin{aligned}
& \mathbb{E}_{\mathbb{Q}_{x, D}^{z, z^{\prime}, \alpha}}\left[\widetilde{M}_{D_{n}^{(u)}}(u, \alpha) \mid N\left(x, r_{\mathfrak{a}_{n}}\right),(1+\varepsilon)^{2} \Upsilon\left(x, r_{\mathfrak{a}_{n}-K}\right)\right] \\
\leq & c_{(4.32)} \mathrm{e}^{-\alpha C_{\mathcal{B}_{\mathfrak{a}_{n}-K}}(u)} \mathrm{e}^{2 \sqrt{\alpha c(4.32)}} .
\end{aligned}
$$


On the event $E^{(x)}\left(r_{0}, \gamma\right), N\left(x, r_{\mathfrak{a}_{n}}\right) \leq \gamma \log \frac{1}{r_{\mathfrak{a}_{n}}}$ and $\Upsilon\left(x, r_{\mathfrak{a}_{n}-K}\right) \leq \gamma\left(\log \frac{1}{r_{\mathfrak{a}_{n}-K}}\right)^{2}$, hence

$$
c_{(4.32)} \leq(1+\varepsilon)^{2} \gamma\left(\log \frac{1}{r_{\mathfrak{a}_{n}-K}}\right)^{2}+2 c_{13}\left(\gamma \log \frac{1}{r_{\mathfrak{a}_{n}}}+1\right) \leq(1+\varepsilon)^{2} \gamma\left(\log \frac{c_{14}}{r_{\mathfrak{a}_{n}}}\right)^{2}
$$

where $c_{14}=c_{14}\left(K, \gamma, r_{0}\right)>0$ denotes some constant in the last inequality. Therefore

$$
\begin{aligned}
& \mathbb{E}_{\mathbb{Q}_{x, D}^{z, z^{\prime}, \alpha}}\left[\widetilde{M}_{D_{n}^{(u)}}(u, \alpha), E^{(x)}\left(r_{0}, \gamma\right)\right] \\
\leq & (1+\varepsilon)^{2} \gamma\left(\log \frac{c_{14}}{r_{\mathfrak{a}_{n}}}\right)^{2} \mathrm{e}^{-\alpha C_{\mathcal{B}_{\mathfrak{a}_{n}-K}}(u)}\left(\frac{c_{14}}{r_{\mathfrak{a}_{n}}}\right)^{2(1+\varepsilon) \sqrt{\alpha \gamma}} .
\end{aligned}
$$

By definition, $C_{\mathcal{B}_{\mathfrak{a}_{n}-K}}(u)=\int_{\mathcal{C}\left(x, r_{\mathfrak{a}_{n}-K}\right)} \log \frac{1}{|u-y|} H_{\mathcal{B}_{\mathfrak{a}_{n}-K}}(u, y) \mathrm{d} y \geq \log \frac{1}{2 r_{\mathfrak{a}_{n}-K}}$ and in the same way, $C_{D_{n}^{(x)}}(u) \geq \log \frac{1}{\sqrt{2} 2^{-n}}$. Using (4.30) yields that uniformly in $u \in D_{n}^{(x)}$,

$$
\begin{aligned}
& \mathbb{E}_{\mathbb{Q}_{x, D}^{z, z^{\prime}, \alpha}}\left[M_{D_{n}^{(u)}}(u, \alpha), E^{(x)}\left(r_{0}, \gamma\right)\right] \\
\leq & c_{15}\left(\log \frac{1}{r_{\mathfrak{a}_{n}}}\right)^{2} \mathrm{e}^{2 \sqrt{2 \alpha \gamma \log \frac{1}{r_{\mathfrak{a}_{n}}}}}\left(\frac{1}{r_{\mathfrak{a}_{n}}}\right)^{2(1+\varepsilon) \sqrt{\alpha \gamma}-\alpha} .
\end{aligned}
$$

Recall that $r_{\mathfrak{a}_{n}}<\frac{1}{4} 2^{-n} \leq r_{\mathfrak{a}_{n}-1}$. We deduce from (4.33) that

$$
\mathbb{E}_{\mathbb{Q}_{x, D}^{z, z^{\prime}, \alpha}}\left[\int_{u \in D_{n}^{(x)}} \frac{\mathcal{M}_{\mathscr{D}_{n}}^{\alpha}(\mathrm{d} u)}{|u-x|^{\varrho}}, E^{(x)}\left(r_{0}, \gamma\right)\right] \rightarrow 0, \quad n \rightarrow \infty .
$$

Then equation (4.18) comes from (4.16), (4.23), (4.27) and (4.34). This completes the proof of Proposition 4.2.

\section{Image of of $\mathcal{M}_{\infty}^{\alpha}$ under a conformal mapping}

The following result is analogous to [1], Theorem 5.2, with the extensions to all $\alpha \in(0,2)$. Recall that $C_{D}(x)=-\int_{\partial D} \log (|x-y|) H_{D}(x, y) \mathrm{d} y$ and $\xi_{D}\left(x, z, z^{\prime}\right)=\frac{2 \pi H_{D}(x, z) H_{D}\left(x, z^{\prime}\right)}{H_{D}\left(z, z^{\prime}\right)}$.

Proposition 5.1 Let $0 \leq \alpha<2$. Recall that $D$ is a simply connected nice domain and $z, z^{\prime}$ nice points of $\bar{D}$. For any nonnegative measurable function $f$, we have

$$
\mathbb{E}_{D}^{z, z^{\prime}} \int_{D} f(x, B) \mathcal{M}_{\infty}^{\alpha}(\mathrm{d} x)=\int_{D} \mathbb{E}_{\mathbb{Q}_{x, D}^{z, z^{\prime}, \alpha}}(f(x, B)) \mathrm{e}^{-\alpha C_{D}(x)} \xi_{D}\left(x, z, z^{\prime}\right) \mathrm{d} x .
$$

Consequently, if for a family of events $\left(E_{x}\right)_{x \in D}, \mathbb{Q}_{x, D}^{z, z^{\prime}, \alpha}\left(E_{x}\right)=1$ for Lebesgue-a.e. $x \in D$, then with probability one, the event $E_{x}$ holds for $\mathcal{M}_{\infty}^{\alpha}$-almost all $x$. 
Proof of Proposition 5.1. By the standard monotone class argument, it is enough to prove the equality $(5.1)$ for $f(x, B)=1_{A}(x) 1_{F}$, where $A \subset D$ a Borel set and $F \in \mathscr{F}_{\mathscr{D}_{n}}$ for an arbitrary $n \geq 1$. Then the left-hand-side of (5.1) is equal to

$$
\begin{aligned}
\mathbb{E}_{D}^{z, z^{\prime}}\left(\mathcal{M}_{\infty}^{\alpha}(A) 1_{F}\right) & =\int_{A} \mathbb{E}_{D}^{z, z^{\prime}}\left(M_{D_{n}^{(x)}}(x, \alpha) 1_{F}\right) \mathrm{d} x \\
& =\int_{A} \mathbb{Q}_{x, D}^{z, z^{\prime}, \alpha}(F) \mathrm{e}^{-\alpha C_{D}(x)} \xi_{D}\left(x, z, z^{\prime}\right) \mathrm{d} x
\end{aligned}
$$

where the first equality follows from Theorem 4.1 and the second from Corollary 3.6.

As pointed out in [1], equation (5.1) characterizes the measure $\mathcal{M}_{\infty}^{\alpha}$. To be more precise, under the assumptions of Proposition 5.1, suppose that there is a random finite measure $\mathfrak{m}$ on the Borel sets of $\mathbb{R}^{2}$, measurable with respect to the Brownian $B:=$ $\left(B_{t}\right)_{0 \leq t \leq T_{z^{\prime}}}$, and which also verifies equation (5.1), replacing $\mathcal{M}_{\infty}^{\alpha}$ by $\mathfrak{m}$ there. Then $\mathfrak{m}=M_{\infty}^{\alpha} \mathbb{P}_{D}^{z, z^{\prime}}$-a.s. To see it, define $\mathfrak{m}^{\prime}:=\mathfrak{m}-M_{\infty}^{\alpha}$. Equation (5.1) applied for $\mathfrak{m}$ and $M_{\infty}^{\alpha}$ yields that, for any bounded measurable function $f$,

$$
\mathbb{E}_{D}^{z, z^{\prime}} \int_{D} f(x, B) \mathfrak{m}^{\prime}(\mathrm{d} x)=0
$$

Let $A$ a Borel set of $\mathbb{R}^{2}$. For $c>0$, taking $f(x, B):=\mathfrak{m}^{\prime}(A) 1_{x \in A} 1_{\left|\mathfrak{m}^{\prime}(A)\right|<c}$, we get that $\mathbb{E}_{D}^{z, z^{\prime}}\left[\mathfrak{m}^{\prime}(A)^{2} 1_{\left|\mathfrak{m}^{\prime}(A)\right|<c}\right]=0$, hence by monotone convergence, $\mathbb{E}_{D}^{z, z^{\prime}}\left[\mathfrak{m}^{\prime}(A)^{2}\right]=0$. We deduce the claim.

Let $D, D^{\prime}$ be simply connected nice domains, and $\Psi: D \rightarrow D^{\prime}$ a conformal transformation. Let $z, z^{\prime}$ be distinct nice points of $\bar{D}$ such that $\Psi(z)$ and $\Psi\left(z^{\prime}\right)$ are nice points of $\overline{D^{\prime}}$ ( $\Psi$ can be extended to a conformal transformation of $D \cup \mathcal{B}(z, \varepsilon) \cup \mathcal{B}\left(z^{\prime}, \varepsilon\right)$ for some $\varepsilon>0$, see Lawler [10], p. 48).

Proposition 5.2 The image measure of $\mathcal{M}_{\infty}^{\alpha}$ by $\Psi$ under $\mathbb{P}_{D}^{z, z^{\prime}}$ has the same law as the measure $\left|\Psi^{\prime}\left(\Psi^{-1}(x)\right)\right|^{-2-\alpha} \mathcal{M}_{\infty}^{\alpha}(\mathrm{d} x)$ under $\mathbb{P}_{D^{\prime}}^{\Psi(z), \Psi\left(z^{\prime}\right)}$.

Proof of Proposition 5.2. It suffices to show that for any nonnegative measurable function $f$,

$$
\begin{aligned}
& \mathbb{E}_{D}^{z, z^{\prime}} \int_{D} f(\Psi(x), \Psi(B)) \mathcal{M}_{\infty}^{\alpha}(\mathrm{d} x) \\
= & \mathbb{E}_{D^{\prime}}^{\Psi(z), \Psi\left(z^{\prime}\right)} \int_{D^{\prime}} f(x, B)\left|\Psi^{\prime}\left(\Psi^{-1}(x)\right)\right|^{-2-\alpha} \mathcal{M}_{\infty}^{\alpha}(\mathrm{d} x) .
\end{aligned}
$$


From Proposition 5.1, the left-hand side is

$$
\int_{D} \mathbb{E}_{\mathbb{Q}_{x, D}^{z, z^{\prime}, \alpha}}(f(\Psi(x), \Psi(B))) \mathrm{e}^{-\alpha C_{D}(x)} \xi_{D}\left(x, z, z^{\prime}\right) \mathrm{d} x .
$$

The conformal invariance of $\mathbb{Q}_{x, D}^{z, z^{\prime}, \alpha}$ (which results from the conformal invariance of the Brownian measures $\mathbb{P}_{D}^{z, x}, \mathbb{P}_{D}^{x, z^{\prime}}$ and $\left.\nu_{D}(x, x)\right)$ implies that

$$
\mathbb{E}_{\mathbb{Q}_{x, D}^{z, z^{\prime}, \alpha}}(f(\Psi(x), \Psi(B)))=\mathbb{E}_{\mathbb{Q}_{\Psi(x), D^{\prime}}^{\Psi(z), \Psi\left(z^{\prime}\right), \alpha}}(f(\Psi(x), B)) .
$$

Moreover, $\xi_{D}\left(x, z, z^{\prime}\right)=\xi_{D^{\prime}}\left(\Psi(x), \Psi(z), \Psi\left(z^{\prime}\right)\right)$ and $C_{D}(x)=C_{D^{\prime}}(\Psi(x))+\log \left|\Psi^{\prime}(x)\right|$. By the change of variables $y=\Psi(x)$, equation (5.3) becomes

$$
\int_{D^{\prime}} \mathbb{E}_{\mathbb{Q}_{y, D^{\prime}}^{\Psi(z),\left(z^{\prime}\right), \alpha}}(f(y, B)) \mathrm{e}^{-\alpha C_{D^{\prime}}(y)} \xi_{D^{\prime}}\left(y, \Psi(z), \Psi\left(z^{\prime}\right)\right)\left|\Psi^{\prime}\left(\Psi^{-1}(y)\right)\right|^{-2-\alpha} \mathrm{d} y
$$

which proves (5.2) by another use of Proposition 5.1.

Proposition 5.3 Let $z, z^{\prime}$ be distinct nice points of $\bar{D}$. We have $\mathbb{P}_{D}^{z, z^{\prime}}\left(\mathcal{M}_{\infty}^{\alpha}(D)>0\right)=1$.

Proof of Proposition 5.3. To stress the dependence of $D$ in $\mathcal{M}_{\infty}^{\alpha}$, we write $\mathcal{M}_{\infty, D}^{\alpha}$ in this proof. We want to prove that $\mathbb{P}_{D}^{z, z^{\prime}}\left(\mathcal{M}_{\infty, D}^{\alpha}=0\right)=0$. By Proposition 5.2, $c:=$ $\mathbb{P}_{D}^{z, z^{\prime}}\left(\mathcal{M}_{\infty, D}^{\alpha}=0\right)$ is independent of $z, z^{\prime}$ and $D$. Consider $D=(0,1)^{2}$, and $\widetilde{D} \in \mathscr{D}_{2}$ a square of mesh $\frac{1}{4}$. The event $\left\{\mathcal{M}_{\infty, D}^{\alpha}=0\right\}$ implies that $\left\{\mathcal{M}_{\infty, D}^{\alpha}(\widetilde{D})=0\right\}$. Conditionally on $\mathscr{F}_{\widetilde{D}}^{+}$and on $y, y^{\prime}$ the starting and ending points of an excursion inside $\widetilde{D}$ (if it exists),

$$
\mathbb{P}_{D}^{z, z^{\prime}}\left(\mathcal{M}_{\infty, D}^{\alpha}(\widetilde{D})=0 \mid \mathscr{F}_{\widetilde{D}}^{+}\right) \leq \mathbb{P}_{\widetilde{D}}^{y, y^{\prime}}\left(\mathcal{M}_{\infty, \widetilde{D}}^{\alpha}=0\right)=c
$$

Let $\mathscr{K}:=\#\left\{\widetilde{D} \in \mathscr{D}_{2}:\right.$ there is an excursion inside $\left.\widetilde{D}\right\}$. It follows that

$$
c=\mathbb{P}_{D}^{z, z^{\prime}}\left(\mathcal{M}_{\infty, D}^{\alpha}(\widetilde{D})=0\right) \leq \mathbb{E}_{D}^{z, z^{\prime}}\left(c^{\mathscr{K}}\right)
$$

Note that $c<1, \mathscr{K} \in\{1,2,3,4\}, \mathbb{P}_{D}^{z, z^{\prime}}$-a.s. and $\mathscr{K} \geq 2$ holds with positive probability. Then the only possibility is that $c=0$.

\section{Thick points}

Recalling $D$ a simply connected nice domain and $z \neq z^{\prime}$ nice points of $\bar{D}$. By using Proposition 5.1, we immediately deduce from Lemma 4.7 (i) the following result. Let $N(x, r)$ be as in (4.10) the number of loops from $x$ that hit $\mathcal{C}(x, r)$. 
Corollary 6.1 Let $0<\alpha<2$. With $\mathbb{P}_{D}^{z, z^{\prime}}$-probability one, the measure $\mathcal{M}_{\infty}^{\alpha}$ is supported on the set of points $x$ such that

$$
\lim _{r \rightarrow 0^{+}} \frac{N(x, r)}{\log 1 / r}=\alpha
$$

Corollary 6.1 is an extension of [1], Corollary 5.1, to all $\alpha \in(0,2)$.

In the sequel, we establish the relationship between $\mathcal{M}_{\infty}^{\alpha}$ and the thick points defined in (1.2).

Theorem 6.2 Let $\alpha \in(0,2)$. With $\mathbb{P}_{D}^{z, z^{\prime}}$-probability one, the measure $\mathcal{M}_{\infty}^{\alpha}$ is supported by the set of $\alpha$-thick points.

By assuming Theorem 6.2, we are able to complete the proof of Theorem 1.1: Proof of Theorem 1.1. By Corollary 6.1 and Theorem 6.2, it remains to check (1.3).

The upper bound of (1.3) follows from that of Theorem B. In fact, for any sufficiently small $r>0$, the law of $\left(B_{T_{\mathcal{C}(z, r) \cap D}+t}, 0 \leq t \leq T_{\mathcal{C}\left(z^{\prime}, r\right) \cap D}-T_{\mathcal{C}(z, r) \cap D}\right)$ under $\mathbb{P}_{D}^{z, z^{\prime}}$ is absolutely continuous with respect to that of a planar Brownian motion. Then we deduce from Theorem B that

$$
\operatorname{dim}_{\mathrm{H}}\{\alpha \text {-thick points }\}=2-\alpha, \quad \mathbb{P}_{D}^{z, z^{\prime}} \text {-a.s. }
$$

which in view of Theorem 6.2 yields the upper bound in (1.3).

For the lower bound in (1.3), let $E^{(x)}\left(r_{0}, \gamma\right)$ be the event defined in (4.17). Corollary 4.3 says that $\mathbb{P}_{D}^{z, z^{\prime}}$-a.s., $\mathcal{M}_{\mathscr{D}_{n}}^{\alpha}$ converges weakly to $\mathcal{M}_{\infty}^{\alpha}$. By Theorem 4.1 , the probability $Q_{D}^{z, z^{\prime}, \alpha}$ defined in (4.3) with $A=D$ there, is absolutely continuous with respect to $\mathbb{P}_{D}^{z, z^{\prime}}$. Hence $\mathcal{M}_{\mathscr{D}_{n}}^{\alpha}$ converges weakly to $\mathcal{M}_{\infty}^{\alpha}, Q_{D}^{z, z^{\prime}, \alpha}$-a.s. In other words, for Lebesgue-a.e. $x \in D$, $\mathbb{Q}_{x, D}^{z, z^{\prime}, \alpha}$-a.s., $\mathcal{M}_{\mathscr{D}_{n}}^{\alpha}$ converges weakly to $\mathcal{M}_{\infty}^{\alpha}$.

Let $0<\varrho<2-\alpha$. Using the same arguments leading to (4.19) and by replacing $\mathcal{M}_{\mathscr{D}_{n}}^{\alpha}(D)$ by $\int_{D} \frac{\mathcal{M}_{\mathscr{D} n}^{\alpha}(\mathrm{d} u)}{|u-x|^{\varrho}}$, we deduce from (4.18) that

$$
\mathbb{E}_{\mathbb{Q}_{x, D}^{z, z^{\prime}, \alpha}}\left[\int_{D} \frac{\mathcal{M}_{\infty}^{\alpha}(\mathrm{d} u)}{|u-x|^{\varrho}}, E^{(x)}\left(r_{0}, \gamma\right)\right] \leq c_{5}\left(r_{0}\right)
$$

for Lebesgue-a.e. $x \in D$.

Let $D^{\left(r_{0}\right)}$ be the set of points $x \in D$ such that $d(x, \partial D)>r_{0}$. It follows from (5.1) 
that for any $r_{0}>0$,

$$
\begin{aligned}
& \mathbb{E}_{D}^{z, z^{\prime}}\left[\int_{D \times D} 1_{E^{(x)}\left(r_{0}, \gamma\right)} \frac{\mathcal{M}_{\infty}^{\alpha}(\mathrm{d} u) \mathcal{M}_{\infty}^{\alpha}(\mathrm{d} x)}{|u-x|^{\varrho}}\right] \\
= & \int_{D} \mathrm{~d} x \mathrm{e}^{-\alpha C_{D}(x)} \xi_{D}\left(x, z, z^{\prime}\right) \mathbb{E}_{\mathbb{Q}_{x, D}^{z, z^{\prime}, \alpha}}\left[\int_{D} \frac{\mathcal{M}_{\infty}^{\alpha}(\mathrm{d} u)}{|u-x|^{\varrho}}, E^{(x)}\left(r_{0}, \gamma\right)\right] \\
\leq & c_{5}\left(r_{0}\right) \int_{D} \mathrm{~d} x \mathrm{e}^{-\alpha C_{D}(x)} \xi_{D}\left(x, z, z^{\prime}\right) \\
< & \infty
\end{aligned}
$$

Hence $\mathbb{P}_{D}^{z, z^{\prime}}$-almost surely, for all $r_{0}>0$,

$$
\int_{D \times D} 1_{E^{(x)}\left(r_{0}, \gamma\right)} \frac{\mathcal{M}_{\infty}^{\alpha}(\mathrm{d} u) \mathcal{M}_{\infty}^{\alpha}(\mathrm{d} x)}{|u-x|^{\varrho}}<\infty
$$

By (4.20) and Proposition 5.1, $\mathbb{P}_{D}^{z, z^{\prime}}$-almost surely, $\mathcal{M}_{\infty}^{\alpha}$-almost all $x, \cup_{\ell=1}^{\infty} E^{(x)}\left(2^{-\ell}, \gamma\right)$ holds. Then by Proposition 5.3, for some $\ell \geq 1$, the measure $\mathfrak{m}_{\ell}(\mathrm{d} x):=1_{E^{(x)}\left(2^{-\ell}, \gamma\right)} \mathcal{M}_{\infty}^{\alpha}(\mathrm{d} x)$ is not trivial and $\int_{D \times D} \frac{\mathfrak{m}_{\ell}(\mathrm{d} u) \mathfrak{m}_{\ell}(\mathrm{d} x)}{|u-x|^{\varrho}}<\infty$.

For any Borel set $A$ such that $\mathcal{M}_{\infty}^{\alpha}\left(A^{c}\right)=0$, we have $\mathfrak{m}_{\ell}\left(A^{c}\right)=0$ and $\int_{A \times A} \frac{\mathfrak{m}_{\ell}(\mathrm{d} u) \mathfrak{m}_{\ell}(\mathrm{d} x)}{|u-x|^{\varrho}}=$ $\int_{D \times D} \frac{\mathfrak{m}_{\ell}(\mathrm{d} u) \mathfrak{m}_{\ell}(\mathrm{d} x)}{|u-x|^{\varrho}}<\infty$. It follows from Frostman's lemma that $\operatorname{dim}_{\mathrm{H}}(A) \geq \varrho$. This yields that a.s., $\operatorname{Dim}\left(\mathcal{M}_{\infty}^{\alpha}\right) \geq \varrho$. As $\varrho$ can be as close as possible to $2-\alpha$, we get the lower bound in (1.3). This completes the proof of Theorem 1.1.

Denote by $\zeta(\mathfrak{e})$ the lifetime of a loop $\mathfrak{e}$ and let

$$
\Lambda_{s}:=\sum_{v \leq s} \zeta\left(\mathfrak{e}_{v}\right), \quad 0 \leq s \leq \alpha
$$

Note that $\Lambda$ is a subordinator on $[0, \alpha]$ whose sample paths are strictly increasing. The law of $\zeta$ under $\nu_{D}(x, x)$ can easily be computed: it follows from (2.12) that

$$
\begin{aligned}
\nu_{D}(x, x)(\zeta \in \mathrm{d} t) & =\pi \mu_{D}(x, x ; t)(\zeta \in \mathrm{d} t) \\
& =\lim _{\varepsilon \rightarrow 0^{+}} \frac{1}{\varepsilon^{2}} \mathbb{P}^{x}\left(t<T_{\partial D},\left|B_{t}-x\right|<\varepsilon\right) \mathrm{d} t \\
& =\pi p_{D}(t, x, x) \mathrm{d} t .
\end{aligned}
$$

Let us define $\left(\ell_{t}^{x}\right)_{t \geq 0}$ by the inverse of $\Lambda$ :

$$
\ell_{t}^{x}:= \begin{cases}0, & \text { if } t \leq T_{x}, \\ \inf \left\{s>0: \Lambda_{s}>t-T_{x}\right\}, & \text { if } T_{x} \leq t<T_{x}+\Lambda_{\alpha}, \\ \alpha, & \text { if } t \geq T_{x}+\Lambda_{\alpha} .\end{cases}
$$


By construction, $\mathbb{Q}_{x, D}^{z, z^{\prime}, \alpha}$-almost surely, $t \rightarrow \ell_{t}^{x}$ is continuous. Moreover, by imitating the proof in the one-dimensional Brownian motion case (for instance the proof of Proposition VI.2.5, [20]), we get that $\mathbb{Q}_{x, D}^{z, z^{\prime}, \alpha}$-almost surely, the support of $\mathrm{d} \ell_{t}^{x}$ is exactly equal to the level set $\left\{T_{x} \leq t \leq T_{x}+\Lambda_{\beta}: B_{t}=x\right\}$. We call $\left(\ell_{t}^{x}\right)_{t \geq 0}$ the family of local times of $B$ at $x$. We mention that after $T_{x}+\Lambda_{\beta}, B$ is a Brownian motion started at $x$ and conditioned to hit $z^{\prime}$. Theorems 6.2 and 1.2 will be a consequence of the following result:

Proposition 6.3 For any $x \in D, \mathbb{Q}_{x, D}^{z, z^{\prime}, \alpha}-$ a.s. for all $0 \leq t \leq T_{z^{\prime}}$,

$$
\lim _{r \rightarrow 0^{+}} \frac{1}{r^{2}(\log r)^{2}} \int_{0}^{t} 1_{\left\{B_{s} \in \mathcal{B}(x, r)\right\}} \mathrm{d} s=\ell_{t}^{x},
$$

where $\ell_{t}^{x}$, defined in (6.3), denotes the local time at $x$ up to time $t$ (under $\mathbb{Q}_{x, D}^{z, z^{\prime}, \alpha}$ ).

By assuming Proposition 6.3 for the moment, we give the proofs of Theorems 6.2 and 1.2:

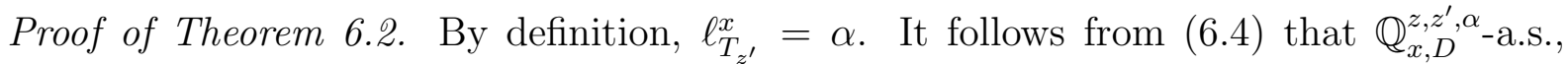
$\lim _{r \rightarrow 0^{+}} \frac{1}{r^{2}(\log r)^{2}} \int_{0}^{T_{z^{\prime}}} 1_{\left\{B_{s} \in \mathcal{B}(x, r)\right\}} \mathrm{d} s=\alpha$, which in view of Proposition 5.1 yields Theorem 6.2 .

Proof of Theorem 1.2. For any $x \in D$, we define

$$
\mathfrak{L}_{t}^{x}:=\lim _{r \rightarrow 0^{+}} \frac{1}{r^{2}(\log r)^{2}} \int_{0}^{t \wedge T_{z^{\prime}}} 1_{\left\{B_{s} \in \mathcal{B}(x, r)\right\}} \mathrm{d} s, \text { if the limit exists for all } t \geq 0,
$$

and $\mathfrak{L}_{t}^{x}:=0, \forall t \geq 0$, otherwise. By Proposition 6.3, for any $x \in D, \mathbb{Q}_{x, D}^{z, z^{\prime}, \alpha}$-a.s., $\mathfrak{L}_{t}^{x}=\ell_{t \wedge T_{z^{\prime}}}^{x}$ for all $t \geq 0$. Then $\mathbb{Q}_{x, D}^{z, z^{\prime}, \alpha}$-a.s., $t \rightarrow \mathfrak{L}_{t}^{x}$ is a continuous additive functional and such that $t \rightarrow \mathrm{d} \mathfrak{L}_{t}^{x}$ is supported by $\left\{t \in\left[0, T_{z^{\prime}}\right]: B_{t}=x\right\}$. By Proposition 5.1, we get Theorem 1.2.

The rest of this section is devoted to the proof of Proposition 6.3. Recall $\Lambda$ defined in (6.1). Let for any $r>0$ and $0 \leq \beta<\alpha$,

$$
I_{\beta}(r):=\int_{0}^{T_{x}+\Lambda_{\beta}} 1_{\left\{B_{s} \in \mathcal{B}(x, r)\right\}} \mathrm{d} s \quad \text { and } \quad I_{\alpha}(r):=\int_{0}^{T_{z^{\prime}}} 1_{\left\{B_{s} \in \mathcal{B}(x, r)\right\}} \mathrm{d} s .
$$

Notice that $I_{0}(r)=\int_{0}^{T_{x}} 1_{\left\{B_{s} \in \mathcal{B}(x, r)\right\}} \mathrm{d} s$ and $I_{\alpha}(r)-I_{\alpha-}(r)=\int_{T_{x}+\Lambda_{\alpha}}^{T_{z^{\prime}}} 1_{\left\{B_{s} \in \mathcal{B}(x, r)\right\}} \mathrm{d} s$ correspond to the times spent in $\mathcal{B}(x, r)$ by a conditioned Brownian motion under $\mathbb{P}_{D}^{z, x}$ and under $\mathbb{P}_{D}^{x, z^{\prime}}$ respectively. In the literature, there is a law of the iterated logarithm for their asymptotics as $r \rightarrow 0^{+}$, see Ray [19] and Le Gall [12]. 
Proof of Proposition 6.3. Proposition 6.3 will follow from the following statement: $\mathbb{Q}_{x, D}^{z, z^{\prime}, \alpha}$-a.s., for all $\beta \in[0, \alpha]$,

$$
\lim _{r \rightarrow 0^{+}} \frac{I_{\beta}(r)}{r^{2}(\log r)^{2}}=\beta
$$

In fact, take $\beta=0$ in (6.5) implies (6.4) for all $t \in\left[0, T_{x}\right]$. For $t \in\left[\Lambda_{\alpha}+T_{x}, T_{z^{\prime}}\right]$, we remark that $I_{\alpha-}(r) \leq \int_{0}^{t} 1_{\left\{B_{s} \in \mathcal{B}(x, r)\right\}} \mathrm{d} s \leq I_{\alpha}(r)$, then by (6.5) we see that (6.4) holds for these $t$. Now consider $T_{x}<t<T_{x}+\Lambda_{\alpha}, \beta:=\ell_{t}^{x} \in(0, \alpha)$. We have $\Lambda_{\beta-}+T_{x} \leq$ $t \leq \Lambda_{\beta}+T_{x}$. Then $I_{\beta-}(r) \leq \int_{0}^{t} 1_{\left\{B_{s} \in \mathcal{B}(x, r)\right\}} \mathrm{d} s \leq I_{\beta}(r)$. Applying (6.5) gives (6.4) for any $T_{x}<t<\Lambda_{\beta}+T_{x}$.

In order to prove (6.5), by the monotonicity, we only need to show that for any fixed $\beta \in(0, \alpha], \mathbb{Q}_{x, D}^{z, z^{\prime}, \alpha}$-a.s.,

$$
\lim _{r \rightarrow 0^{+}} \frac{I_{\beta}(r)}{r^{2}(\log r)^{2}}=\beta
$$

To this end, let $r_{0}>0$ such that $\mathcal{B}\left(x, r_{0}\right) \subset D \backslash\left\{z, z^{\prime}\right\}$. For any $0<r<r_{0}$, denote by $L(r)$ the local time at $\mathcal{C}(x, r)$ of $\left(B_{t}\right)_{t \geq 0}$ (as the occupation time density at $r$ of the process $\left.\left(\left|B_{t}-x\right|\right)_{t \geq 0}\right)$ till $T_{x}+\Lambda_{\beta}$ if $\beta<\alpha$ and till $T_{z^{\prime}}$ if $\beta=\alpha$. Then

$$
I_{\beta}(r)=\int_{0}^{r} L(u) \mathrm{d} u .
$$

We will prove that $\mathbb{Q}_{x, D}^{z, z^{\prime}, \alpha}$-a.s.,

$$
\lim _{r \rightarrow 0^{+}} \frac{L(r)}{r(\log r)^{2}}=2 \beta,
$$

which gives (6.6) by integrating $L(\cdot)$.

Without loss of generality, we suppose that $x=0$. We can decompose the trajectories in $\mathcal{B}\left(0, r_{0}\right)$ as

- Brownian excursions from $\mathcal{C}\left(0, r_{0}\right)$ to $\mathcal{C}\left(0, r_{0}\right)$ which do not hit 0 ,

- Brownian excursions from $\mathcal{C}\left(0, r_{0}\right)$ to 0 or from 0 to $\mathcal{C}\left(0, r_{0}\right)$,

- and Brownian loops at 0 in $\mathcal{B}\left(0, r_{0}\right)$.

The Brownian excursions which do not hit 0 can be ignored to understand the asymptotics of the local time around 0 since none will hit $\mathcal{C}(0, r)$ for $r$ small enough.

Let us consider the Brownian excursions from 0 to $\mathcal{C}\left(0, r_{0}\right)$. These excursions are of finite number almost surely. The norm of an excursion is a two-dimensional Bessel process stopped at the first hitting time of $r_{0}$. Its local time process at $r \in\left(0, r_{0}\right)$ is 
equal in law to the process $\left(r U_{\log \left(r_{0} / r\right)}\right)_{r \in\left(0, r_{0}\right)}$ where $U$. is the square of Bessel processes of dimension 2 starting from 0 (see Exercise 2.6, Chapter XI in Revuz and Yor [20]). By the classical law of the iterated logarithm for the process $U$, almost surely as $r \rightarrow 0^{+}$, $U_{\log (1 / r)}=O(\log (1 / r)(\log \log \log 1 / r))$. Hence the contribution to $L(r)$ by the excursions from 0 to $\mathcal{C}\left(0, r_{0}\right)$ are $o\left(r(\log r)^{2}\right)$. The same is true, by time-reversal, of excursions from $\mathcal{C}\left(0, r_{0}\right)$ to 0 .

If we denote by $L_{(\mathfrak{e} .)}(r)$ the local time at $\mathcal{C}(0, r)$ of Brownian loops $\left(\mathfrak{e}_{s}\right)_{s \leq \alpha}$ in $\mathcal{B}\left(0, r_{0}\right)$, then we have shown that almost surely, $L(r)-L_{(\mathfrak{e} .)}(r)=o\left(r(\log r)^{2}\right)$ as $r \rightarrow 0^{+}$. It remains to prove that almost surely, $\lim _{r \rightarrow 0^{+}} \frac{L_{(\mathfrak{e})}(r)}{r(\log r)^{2}}=2 \beta$, or equivalently

$$
\lim _{t \rightarrow \infty} \frac{\mathrm{e}^{t} L_{(\mathfrak{e} .)}\left(\mathrm{e}^{-t}\right)}{t^{2}}=2 \beta
$$

By scaling, we may take $r_{0}=1$. For a loop $\mathfrak{e}$, denote by $h(\mathfrak{e}):=\max _{u \geq 0}|\mathfrak{e}(u)|$ its maximum norm (recalling that $x=0$ ). By Lemma 2.3 (ii), we get that

$$
\nu_{\mathcal{B}(0,1)}(0,0)(h(\mathfrak{e})>r)=\log (1 / r), \quad 0<r \leq 1,
$$

which implies that the point process $\mathcal{P}(\beta):=\sum_{s \leq \beta: \mathfrak{e}_{s} \in \mathcal{B}(0,1)} \delta_{\left\{\log \left(1 / h\left(\mathfrak{e}_{s}\right)\right)\right\}}$ is a Poisson point process on $(0, \infty)$ with intensity $\beta \mathrm{d} t$. Write $0<\mathfrak{u}_{1}<\mathfrak{u}_{2}<\ldots<\mathfrak{u}_{k}<\ldots$ for the points of $\mathcal{P}(\beta)$ and let $N_{t}:=\sum_{k=1}^{\infty} 1_{\left\{\mathfrak{u}_{k} \leq t\right\}}, t \geq 0$. Then $\left(N_{t}\right)_{t \geq 0}$ is a Poisson process with parameter $\beta$.

Conditionally on $\{h(\mathfrak{e})=r\}$, the norm of a Brownian loop $|\mathfrak{e}(\cdot)|$ can be decomposed as a two-dimensional Bessel process from 0 to its first hitting time of $r$, followed by an independent copy of the same process going backwards in time, see Pitman and Yor [18]. Therefore, conditionally on $\left\{h(\mathfrak{e})=\mathrm{e}^{-s}\right\}$, the process of local times at $\mathrm{e}^{-t}$ of $|\mathfrak{e}(\cdot)|$, for $t \geq s$, has the law of the sum of two independent copies of $\left(\mathrm{e}^{-t} U_{t-s}\right)_{t \geq s}$ which by the additivity of the square of Bessel processes, is equal in law to the process $\left(\mathrm{e}^{-t} U_{t-s}^{(4)}\right)_{t \geq s}$, where $U^{(4)}$ denotes the square of Bessel processes of dimension 4, starting from 0 .

Let $U_{k}^{(4)}, k \geq 1$, be an i.i.d. copies of $U^{(4)}$, independent of $\left\{\mathfrak{u}_{k}\right\}_{k \geq 1}$. Then the process $\left(\mathrm{e}^{t} L_{(\mathfrak{e} .)}\left(\mathrm{e}^{-t}\right)\right)_{t \geq 0}$ is equal in law to the process $\left(X_{t}\right)_{t \geq 0}$ where

$$
X_{t}:=\sum_{k=1}^{\infty} 1_{\left\{\mathfrak{u}_{k} \leq t\right\}} U_{t-\mathfrak{u}_{k}}^{(k)}, \quad t \geq 0 .
$$

It is enough to show that almost surely,

$$
\lim _{t \rightarrow \infty} \frac{X_{t}}{t^{2}}=2 \beta .
$$


To this end, write

$$
X_{t}=4 \sum_{k=1}^{\infty}\left(t-\mathfrak{u}_{k}\right) 1_{\left\{\mathfrak{u}_{k} \leq t\right\}}+\widehat{X}_{t}=4 \int_{(0, t]}(t-s) \mathrm{d} N_{s}+\widehat{X}_{t},
$$

where $\widehat{X}_{t}:=\sum_{k=1}^{\infty} 1_{\left\{\mathfrak{u}_{k} \leq t\right\}}\left(U_{t-\mathfrak{u}_{k}}^{(k)}-4\left(t-\mathfrak{u}_{k}\right)\right)$. Notice that $\int_{(0, t]}(t-s) \mathrm{d} N_{s}=\int_{(0, t]} N_{s} \mathrm{~d} s$. By the law of large numbers $\lim _{s \rightarrow \infty} \frac{N_{s}}{s}=\beta$, which yields that $\frac{4}{t^{2}} \int_{(0, t]}(t-s) \mathrm{d} N_{s} \rightarrow 2 \beta$ a.s.

To complete the proof, we only need to check that $\widehat{X}_{t}=o\left(t^{2}\right)$ a.s. It is well-known that $U_{s}-4 s$ is a martingale and $\operatorname{Var}\left(U_{s}\right)=8 s^{2}$ (see Chapter XI in Revuz-Yor [20]). Conditionally on $\left\{\mathfrak{u}_{k}\right\}_{k \geq 1}, \widehat{X}$ is a (finite) sum of independent martingale hence is a martingale. By Doob's $L^{2}$-inequality, we get that for any $t>0$,

$$
\mathbb{E}\left(\sup _{0 \leq s \leq t} \widehat{X}_{s}^{2}\right) \leq 4 \mathbb{E}\left(\widehat{X}_{t}^{2}\right)=32 \mathbb{E} \sum_{k=1}^{\infty} 1_{\left\{\mathfrak{u}_{k} \leq t\right\}}\left(t-\mathfrak{u}_{k}\right)^{2}=\frac{32 \beta}{3} t^{3} .
$$

Using the Borel-Cantelli lemma gives that almost surely, $\sup _{0 \leq s \leq t}\left|\widehat{X}_{s}\right|=O\left(t^{3 / 2+o(1)}\right)$ as $t \rightarrow \infty$. Hence $\widehat{X}_{t}=o\left(t^{2}\right)$ a.s., which completes the proof of Proposition 6.3.

\section{$7 \quad$ Further discussions}

\subsection{The case $\alpha=0$}

In the case $\alpha=0, \mathbb{Q}_{x, D}^{z, z^{\prime}, \alpha}$ is the law of a Brownian excursion from $z$ to $x$ in $D$, followed by an independent Brownian excursion from $x$ to $z^{\prime}$ in $D$. We claim that for any $z, z^{\prime}$ two distinct nice points of $\bar{D}$,

$$
\mathcal{M}_{\infty}^{0}(\bullet)=\pi \mu(\bullet \cap D), \quad \mathbb{P}_{D}^{z, z^{\prime}} \text {-a.s. }
$$

where $\mu$ denotes the occupation measure of the Brownian motion (under $\mathbb{P}_{D}^{z, z^{\prime}}$ ) defined by $\mu(A):=\int_{0}^{T_{z^{\prime}}} 1_{\left\{B_{t} \in A\right\}} \mathrm{d} t$ for any Borel set $A$. To identify the two measures, we only need to show that

$$
\pi \mathbb{E}_{D}^{z, z^{\prime}} \int_{D} f(x, B) \mu(\mathrm{d} x)=\int_{D} \mathbb{E}_{\mathbb{Q}_{x, D}^{z, z^{\prime}, 0}}(f(x, B)) \xi_{D}\left(x, z, z^{\prime}\right) \mathrm{d} x,
$$

for $f(x, B)=\tilde{h}(x) \mathrm{e}^{-\int_{0}^{\infty} h\left(t, B_{t}\right) d t}$ with nonnegative Borel functions $h$ and $\tilde{h}$.

It is enough to prove (7.2) for $z \in D$; the case when $z \in \partial D$ follows by considering $z_{n}:=z+\varepsilon_{n} \mathbf{n}_{z}$ with $\varepsilon_{n} \rightarrow 0^{+}$as $n \rightarrow \infty$.

Consider the right-hand side of (7.2). By the strong Markov property at time $T_{x}$,

$$
\mathbb{E}_{\mathbb{Q}_{x, D}^{z, z^{\prime}, 0}}(f(x, B))=\tilde{h}(x) \mathbb{E}_{D}^{z, x}\left[\mathrm{e}^{-\int_{0}^{T_{x}} h\left(t, B_{t}\right) \mathrm{d} t}\right] \mathbb{E}_{D}^{x, z^{\prime}}\left[\mathrm{e}^{-\int_{0}^{T_{z^{\prime}}} h\left(t, B_{t}\right) \mathrm{d} t}\right] .
$$


The law $\mathbb{P}_{D}^{z, x}$ is the normalized excursion measure denoted by $\mu_{D}^{\#}(z, x)$ in Lawler ([10], Chapter 5.2). It is $\mu_{D}(z, x)$ divided by the mass $2 H_{D}(z, x)$, and

$$
\mu_{D}(z, x):=\int_{0}^{\infty} \mu_{D}(z, x ; s) \mathrm{d} s,
$$

where $\mu_{D}(z, x ; s)$ denotes the measure on the Brownian paths in $D$ of length $s$, from $z$ to $x$ (the factor 2 comes from the renormalization of $H_{D}$ in (2.4)). We refer to Lawler ([10], Chapter 5.2) for the precise definition of $\mu_{D}(z, x ; s)$ and the following equality: For any nonnegative Borel function $F$ and for any $s>0$,

$$
\int_{D} \mu_{D}(z, x ; s)\left(F\left(B_{s}\right) \mathrm{e}^{-\int_{0}^{s} h\left(t, B_{t}\right) \mathrm{d} t}\right) \mathrm{d} x=\mathbb{E}^{z}\left[F\left(B_{s}\right) \mathrm{e}^{-\int_{0}^{s} h\left(t, B_{t}\right) \mathrm{d} t}, s<T_{\partial D}\right] .
$$

It follows that

$$
\begin{aligned}
& \int_{D} F(x) \mathbb{E}_{D}^{z, x}\left[\mathrm{e}^{-\int_{0}^{T_{x}} h\left(t, B_{t}\right) \mathrm{d} t}\right] \mathrm{d} x \\
= & \int_{D} \frac{F(x)}{2 H_{D}(z, x)} \mu_{D}(z, x)\left(\mathrm{e}^{-\int_{0}^{T_{x}} h\left(t, B_{t}\right) \mathrm{d} t}\right) \mathrm{d} x \\
= & \mathbb{E}_{D}^{z}\left[\int_{0}^{T_{\partial D}} \frac{F\left(B_{s}\right)}{2 H_{D}\left(z, B_{s}\right)} \mathrm{e}^{-\int_{0}^{s} h\left(t, B_{t}\right) \mathrm{d} t} \mathrm{~d} s\right],
\end{aligned}
$$

which implies that, with $g(x):=\mathbb{E}_{D}^{x, z^{\prime}}\left[\mathrm{e}^{-\int_{0}^{T_{z^{\prime}}} h\left(t, B_{t}\right) \mathrm{d} t}\right]$,

$$
\begin{aligned}
& \int_{D} \mathbb{E}_{\mathbb{Q}_{x, D}^{z, z^{\prime}, 0}}(f(x, B)) \xi_{D}\left(x, z, z^{\prime}\right) \mathrm{d} x \\
= & \pi \mathbb{E}_{D}^{z}\left[\int_{0}^{T_{\partial D}} \tilde{h}\left(B_{s}\right) g\left(B_{s}\right) \mathrm{e}^{-\int_{0}^{s} h\left(t, B_{t}\right) \mathrm{d} t} \frac{H_{D}\left(B_{s}, z^{\prime}\right)}{H_{D}\left(z, z^{\prime}\right)} \mathrm{d} s\right] .
\end{aligned}
$$

It is, by the $h$-transform (2.7),

$$
\begin{aligned}
& \pi \mathbb{E}_{D}^{z, z^{\prime}}\left[\int_{0}^{T_{z^{\prime}}} \tilde{h}\left(B_{s}\right) g\left(B_{s}\right) \mathrm{e}^{-\int_{0}^{s} h\left(t, B_{t}\right) \mathrm{d} t} \mathrm{~d} s\right] \\
= & \pi \mathbb{E}_{D}^{z, z^{\prime}}\left[\int_{0}^{T_{z^{\prime}}} \tilde{h}\left(B_{s}\right) \mathrm{e}^{-\int_{0}^{T} z^{\prime} h\left(t, B_{t}\right) \mathrm{d} t} \mathrm{~d} s\right] \\
= & \pi \mathbb{E}_{D}^{z, z^{\prime}}\left[\int_{0}^{T_{z^{\prime}}} f\left(B_{s}, B\right) \mathrm{d} s\right],
\end{aligned}
$$

where the first equality follows from the Markov property at time $s$. This proves (7.2). 


\subsection{The intersection local times}

Fix $p \geq 2$ an integer. We take $\alpha_{1}, \alpha_{2}, \ldots, \alpha_{p} \geq 0$ such that $\sum_{i=1}^{p} \alpha_{i}<2$ and $p$ pairs of distinct points $\left(z_{1}, z_{1}^{\prime}\right), \ldots,\left(z_{p}, z_{p}^{\prime}\right)$ in $\bar{D}$. The law of $p$ independent excursions $B^{(i)}$, $1 \leq i \leq p$, inside $D$, from $z_{i}$ to $z_{i}^{\prime}$ is denoted by $\otimes_{i=1}^{p} \mathbb{P}_{D}^{z_{i}, z_{i}^{\prime}}$. For any $x \in D$, we consider $\otimes_{i=1}^{p} \mathbb{Q}_{x, D}^{z_{i}, z_{i}^{\prime}, \alpha_{i}}$ the product measure.

Recall the notation $M_{D_{n}^{(x)}}\left(x, \alpha_{i}\right)$ in Section 4. For any Borel set $A$, the Radon-Nykodim derivative of $\int_{A} \otimes_{i=1}^{p} \mathbb{Q}_{x, D}^{z_{i}, z_{i}^{\prime}, \alpha_{i}} \prod_{i=1}^{p} M_{D}^{(i)}\left(x, \alpha_{i}\right) \mathrm{d} x$ with respect to $\otimes_{i=1}^{p} \mathbb{P}_{D}^{z_{i}, z_{i}^{\prime}}$ on the $\sigma$-field $\mathscr{F}_{\mathscr{D}_{n}}$ is given by

$$
\mathcal{M}_{\mathscr{D}_{n}}^{\alpha_{1}, \ldots, \alpha_{p}}(A):=\int_{A} \prod_{i=1}^{p} M_{D_{n}^{(x)}}^{(i)}\left(x, \alpha_{i}\right) \mathrm{d} x
$$

where $M_{D_{n}^{(x)}}^{(i)}\left(x, \alpha_{i}\right)$ is associated to the trajectory $B^{(i)}$. It is a nonnegative martingale (in $n$, adapted to the filtration $\left.\left(\mathscr{F}_{\mathscr{D}_{n}}\right)_{n \geq 0}\right)$ hence has an almost sure limit $\mathcal{M}_{\infty}^{\alpha_{1}, \ldots, \alpha_{p}}$. We want to show the uniform integrability of this martingale. We need to follow the proof of Proposition 4.2 in Section 4.2.

We let $0 \leq \varrho<2-\sum_{i=1}^{p} \alpha_{i}$. Take $\gamma_{i}=\gamma_{i}(\varrho), i=1 \ldots p$ and $\varepsilon=\varepsilon(\varrho)$ such that $\gamma_{i}>\alpha_{i}$, $\varepsilon>0$ and

$$
\varrho+\sum_{i=1}^{p} 2(1+\varepsilon) \sqrt{\gamma_{i} \alpha_{i}}-\alpha_{i}<2
$$

We keep the notation $\eta, K, r_{0} \in(0,1)$ and $r_{k}=\frac{r_{0}}{2^{k}}$. We define $E^{(x), i}\left(r_{0}, \gamma_{i}\right)$ as in equation (4.17), associated to the trajectory $B^{(i)}$ and the parameters $\alpha_{i}, \gamma_{i}$. We let $E^{(x)}\left(r_{0}, \gamma\right):=\bigcap_{i=1}^{p} E^{(x), i}\left(r_{0}, \gamma_{i}\right)$. We will prove the analog of equation (4.18):

$$
\limsup _{n \rightarrow+\infty} 1_{\left\{d\left(x, \partial D_{n}^{(x)}\right) \geq \frac{2^{-n}}{4}\right\}} \mathbb{E}_{\otimes_{i=1}^{p} \mathbb{Q}_{x, D}^{z_{i}, z_{i}^{\prime}, \alpha_{i}}}\left[\int_{D} \frac{\mathcal{M}_{\mathscr{D}_{n}, \ldots, \alpha_{p}}^{\alpha_{1}}(\mathrm{~d} u)}{|u-x|^{\varrho}}, E^{(x)}\left(r_{0}, \gamma\right)\right] \leq c_{5}\left(r_{0}\right) .
$$

The case $\varrho=0$ implies uniform integrability. We have

$$
\begin{aligned}
& \mathbb{E}_{\otimes_{i=1}^{p} \mathbb{Q}_{x, D}^{z_{i}, z_{i}^{\prime}, \alpha_{i}}}\left[\int_{D} \frac{\mathcal{M}_{\mathscr{D}_{n}}^{\alpha_{1}, \ldots, \alpha_{p}}(\mathrm{~d} u)}{|u-x|^{\varrho}}, E^{(x)}\left(r_{0}, \gamma\right)\right] \\
= & \int_{D} \prod_{i=1}^{p} \mathbb{E}_{\mathbb{Q}_{x, D}^{z_{i}, z_{i}^{\prime}, \alpha_{i}}}\left[\frac{M_{D_{n}^{(u)}}^{(i)}\left(u, \alpha_{i}\right)}{|u-x|^{\varrho}}, E^{(x), i}\left(r_{0}, \gamma_{i}\right)\right] \mathrm{d} u .
\end{aligned}
$$

We now use for each trajectory $B^{(i)}$ :

- if $|u-x|>r_{K}$, the equation before equation (4.23):

$$
\mathbb{E}_{\mathbb{Q}_{x, D}^{z_{i}, z_{i}^{\prime}, \alpha_{i}}}\left[M_{D_{n}^{(u)}}^{(i)}\left(u, \alpha_{i}\right), E^{(x), \alpha_{i}}\left(r_{0}, \gamma_{i}\right)\right] \leq f_{z_{i}, z_{i}^{\prime}}(u) \mathrm{e}^{-\alpha_{i} C_{D}(u)} \mathrm{e}^{2 \sqrt{\alpha_{i} f_{z_{i}, z_{i}^{\prime}}(u)}}
$$


- if $r_{k+K+1} \leq|u-x|<r_{k+K}$ for some $k \geq 0$, with $u \notin D_{n}^{(x)}$, equation (4.27):

$$
\mathbb{E}_{\mathbb{Q}_{x, D}^{z_{i}, z_{i}^{\prime}, \alpha_{i}}}\left[M_{D_{n}^{(u)}}^{(i)}\left(u, \alpha_{i}\right), E^{(x)}\left(r_{0}, \gamma_{i}\right)\right] \leq \frac{c_{11}}{|u-x|^{2(1+\varepsilon) \sqrt{\alpha_{i} \gamma_{i}}-\alpha_{i}}}\left(\log \frac{1}{|u-x|}\right)^{2} .
$$

- if $u \in D_{n}^{(x)}$, equation (4.33), (where $\mathfrak{a}_{n}$ is the smallest integer $j \geq K$ such that $\left.r_{j}<\frac{2^{-n}}{4}\right):$

$$
\begin{aligned}
& \mathbb{E}_{\mathbb{Q}_{x, D}^{z_{i}, z_{i}^{\prime}, \alpha_{i}}}\left[M_{D_{n}^{(u)}}^{(i)}\left(u, \alpha_{i}\right), E^{(x)}\left(r_{0}, \gamma_{i}\right)\right] \\
\leq & c_{15}\left(\log \frac{1}{r_{\mathfrak{a}_{n}}}\right)^{2} \mathrm{e}^{2 \sqrt{2 \alpha_{i} \gamma_{i} \log \frac{1}{r_{\mathfrak{a}_{n}}}}}\left(\frac{1}{r_{\mathfrak{a}_{n}}}\right)^{2(1+\varepsilon) \sqrt{\alpha_{i} \gamma_{i}}-\alpha_{i}} .
\end{aligned}
$$

We can then conclude by integrating over $u$.

By construction, the measure $\mathcal{M}_{\infty}^{\alpha_{1}, \ldots, \alpha_{p}}$ satisfies that for any nonnegative measurable functions $f_{i}, 1 \leq i \leq p$,

$$
\begin{aligned}
& \otimes_{i=1}^{p} \mathbb{E}_{D}^{z_{i}, z_{i}^{\prime}} \int_{D} \prod_{i=1}^{p} f_{i}\left(x, B^{(i)}\right) \mathcal{M}_{\infty}^{\alpha_{1}, \ldots, \alpha_{p}}(\mathrm{~d} x) \\
= & \int_{D} \mathbb{E}_{\otimes_{i=1}^{p} \mathbb{Q}_{x, D}^{z_{i}, z_{i}^{\prime}, \alpha_{i}}}\left(\prod_{i=1}^{p} f_{i}\left(x, B^{(i)}\right)\right) \mathrm{e}^{-C_{D}(x) \sum_{i=1}^{p} \alpha_{i}} \prod_{i=1}^{p} \xi_{D}\left(x, z_{i}, z_{i}^{\prime}\right) \mathrm{d} x .
\end{aligned}
$$

We refer to Le Gall ([14], Chapter 9, Theorem 1) for a similar decomposition involving the self-intersection local times. The measure $\mathcal{M}_{\infty}^{\alpha_{1}, \ldots, \alpha_{p}}$ was constructed by other means by Jego [9], see Proposition 1.2 there.

When $\alpha_{1}=\ldots=\alpha_{p}=0, \pi^{-p} \mathcal{M}_{\infty}^{0, \ldots, 0}$ is nothing else than the image of the $p$-th intersection local times measure. In fact, let $\gamma\left(\mathrm{d} s_{1} \ldots \mathrm{d} s_{p}\right)$ be the intersection local times of $p$ independent excursions $B^{(1)}, \ldots, B^{(p)}$, which means that $\gamma$ is a measure supported by $\left\{\left(s_{1}, \ldots, s_{p}\right) \in \mathbb{R}_{+}^{p}: B_{s_{1}}^{(1)}=\ldots=B_{s_{p}}^{(p)}\right\}$ and formally defined by

$$
\gamma\left(\mathrm{d} s_{1} \ldots \mathrm{d} s_{p}\right):=\delta_{\{0\}}\left(B_{s_{1}}^{(1)}-B_{s_{2}}^{(2)}\right) \cdots \delta_{\{0\}}\left(B_{s_{p-1}}^{(p-1)}-B_{s_{p}}^{(p)}\right) \mathrm{d} s_{1} \cdots \mathrm{d} s_{p} .
$$

Then we may check as in the previous subsection that $\otimes_{i=1}^{p} \mathbb{P}_{D}^{z_{i}, z_{i}^{\prime}}$-a.s., $\pi^{-p} \mathcal{M}_{\infty}^{0, \ldots, 0}$ is exactly the image of $\gamma$ by the application $\left(s_{1}, \ldots ., s_{p}\right) \rightarrow B_{s_{1}}^{(1)}$. The details are omitted. 


\section{A Appendix}

\section{A.1 Justification of (2.8)}

The existence of the limiting probability measure $\mathbb{P}_{D}^{z, z^{\prime}}$ follows from the Kolmogorov extension theorem once we have proven that

$$
\lim _{\varepsilon \rightarrow 0^{+}} \mathbb{E}_{D}^{z+\varepsilon \mathbf{n}_{z}, z^{\prime}}\left(F\left(B_{T_{D \cap \mathcal{C}(z, r)+t}}, t \geq 0\right)\right) \quad \text { exists }
$$

for any $0<r<\left|z-z^{\prime}\right|$ and for any bounded measurable function $F$ on $\mathcal{K}$.

Let $\nu_{D}(z)$ be the excursion measure in $D$ at $z$ defined by

$$
\nu_{D}(z):=\lim _{\varepsilon \rightarrow 0^{+}} \frac{1}{\varepsilon} \mathbb{P}_{D}^{z+\varepsilon \mathbf{n}_{z}},
$$

in the sense that for any $r>0$ such that $D \cap \mathcal{C}(z, r) \neq \emptyset$, any $\mathbf{A} \in \sigma\left\{B_{T_{D \cap \mathcal{C}(z, r)}+t}, t \geq 0\right\}$,

$$
\nu_{D}(z)\left(\mathbf{A} \cap\left\{T_{D \cap \mathcal{C}(z, r)}<T_{\partial D}\right\}\right)=\lim _{\varepsilon \rightarrow 0^{+}} \frac{1}{\varepsilon} \mathbb{P}_{D}^{z+\varepsilon \mathbf{n}_{z}}\left(\mathbf{A} \cap\left\{T_{D \cap \mathcal{C}(z, r)}<T_{\partial D}\right\}\right)
$$

We refer to Burdzy ([3], Theorem 4.1 and p. 34-35) for a justification of (A.3) and the fact that $\nu_{D}(z)\left(T_{D \cap \mathcal{C}(z, r)}<T_{\partial D}\right)<\infty$. By linearity, (A.3) is true when replacing $1_{\mathbf{A}}$ by simple functions, then by any bounded measurable function (any measurable bounded function being limit of simple functions for the uniform norm).

Now let $0<r<\left|z-z^{\prime}\right|$. Using the strong Markov property at time $T_{D \cap \mathcal{C}(z, r)}$ it is enough to show (A.1) for $f\left(B_{T_{D \cap \mathcal{C}(z, r)}}\right)$ instead of $F\left(B_{T_{D \cap \mathcal{C}(z, r)+t}}, t \geq 0\right)$, where $f$ denotes some bounded Borel function.

To this end, we have by the definition of $\mathbb{P}_{D}^{y, z^{\prime}}$ in $(2.7)$ that for any $0<r^{\prime}<\left|z-z^{\prime}\right|-r$,

$$
\begin{aligned}
& \lim _{\varepsilon \rightarrow 0^{+}} \mathbb{E}_{D}^{z+\varepsilon \mathbf{n}_{z}, z^{\prime}}\left(f\left(B_{\left.T_{D \cap \mathcal{C}(z, r)}\right)}\right)\right) \\
& =\lim _{\varepsilon \rightarrow 0^{+}} \mathbb{E}_{D}^{z+\varepsilon \mathbf{n}_{z}}\left[\frac{H_{D}\left(B_{T_{D \cap \mathcal{C}\left(z^{\prime}, r^{\prime}\right)}}, z^{\prime}\right)}{H_{D}\left(z+\varepsilon \mathbf{n}_{z}, z^{\prime}\right)} f\left(B_{\left.T_{D \cap \mathcal{C}(z, r)}\right)}\right), T_{\mathcal{C}\left(z^{\prime}, r^{\prime}\right)}<T_{\partial D}\right] \\
& =\lim _{\varepsilon \rightarrow 0^{+}} \frac{1}{\varepsilon H_{D}\left(z, z^{\prime}\right)} \mathbb{E}_{D}^{z+\varepsilon \mathbf{n}_{z}}\left[H_{D}\left(B_{T_{D \cap \mathcal{C}\left(z^{\prime}, r^{\prime}\right)}}, z^{\prime}\right) f\left(B_{\left.T_{D \cap \mathcal{C}(z, r)}\right)}\right) T_{\mathcal{C}\left(z^{\prime}, r^{\prime}\right)}<T_{\partial D}\right] \\
& =\frac{1}{H_{D}\left(z, z^{\prime}\right)} \nu_{D}(z)\left[H_{D}\left(B_{T_{D \cap \mathcal{C}\left(z^{\prime}, r^{\prime}\right)}}, z^{\prime}\right) f\left(B_{T_{D \cap \mathcal{C}(z, r)}}\right), T_{\mathcal{C}\left(z^{\prime}, r^{\prime}\right)}<T_{\partial D}\right]
\end{aligned}
$$

by (A.2). This completes the proof of (A.1) and justifies the definition of $\mathbb{P}_{D}^{z, z^{\prime}}$ in $(2.8)$. 


\section{A.2 Justification of $(2.14)$}

Let $r>0$ be small such that $\mathcal{B}(x, r) \subset D$ and let $\Phi$ be a bounded measurable function on $\mathcal{K}$. By the strong Markov property at $T_{\mathcal{C}(x, r)}$,

$$
\mathbb{E}_{D}^{z, x}\left[\Phi\left(B_{T_{\mathcal{C}(x, r)}+s}, s \geq 0\right), T_{\mathcal{C}(x, r)}<T_{x}\right]=\mathbb{E}_{D}^{z, x}\left[f\left(B_{T_{\mathcal{C}(x, r)}}\right), T_{\mathcal{C}(x, r)}<T_{x}\right],
$$

with $f(b):=\mathbb{E}_{x, D}^{b}\left[\Phi\left(B_{s}, s \geq 0\right)\right]$. By the $h$-transform,

$$
\begin{aligned}
& \mathbb{E}_{D}^{z, x}\left[f\left(B_{T_{\mathcal{C}(x, r)}}\right), T_{\mathcal{C}(x, r)}<T_{x}\right] \\
= & \lim _{\varepsilon \rightarrow 0^{+}} \mathbb{E}_{D}^{z, x}\left[f\left(B_{T_{\mathcal{C}(x, r)}}\right), T_{\mathcal{C}(x, r)}<T_{\mathcal{C}(x, \varepsilon)}\right] \\
= & \lim _{\varepsilon \rightarrow 0^{+}} \mathbb{E}^{z}\left[\frac{G_{D}\left(x, B_{T_{\mathcal{C}(x, r)}}\right)}{G_{D}(x, z)} f\left(B_{T_{\mathcal{C}(x, r)}}\right), T_{\mathcal{C}(x, r)}<T_{\mathcal{C}(x, \varepsilon)}\right] \\
= & \mathbb{E}^{z}\left[\frac{G_{D}\left(x, B_{T_{\mathcal{C}(x, r)}}\right)}{G_{D}(x, z)} f\left(B_{T_{\mathcal{C}(x, r)}}\right)\right] .
\end{aligned}
$$

It follows that

$$
\begin{aligned}
& \lim _{z \rightarrow x}\left(\log \frac{1}{|z-x|}\right) \mathbb{E}_{D}^{z, x}\left[\Phi\left(B_{\left.T_{\mathcal{C}(x, r)}+\cdot\right)}\right), T_{\mathcal{C}(x, r)}<T_{x}\right] \\
= & \mathbb{E}^{z}\left[G_{D}\left(x, B_{T_{\mathcal{C}(x, r)}}\right) f\left(B_{T_{\mathcal{C}(x, r)}}\right)\right] .
\end{aligned}
$$

Therefore the proof of (2.14) reduces to show that

$$
\nu_{D}(x, x)\left[\Phi\left(\mathfrak{e}_{T_{\mathcal{C}(x, r)}+}\right), T_{\mathcal{C}(x, r)}<T_{x}\right]=\mathbb{E}^{z}\left[G_{D}\left(x, B_{\left.T_{\mathcal{C}(x, r)}\right)}\right) f\left(B_{T_{\mathcal{C}(x, r)}}\right)\right],
$$

where as before $f(b):=\mathbb{E}_{x, D}^{b}[\Phi(B)]$ for any $b \in \mathcal{C}(x, r)$. By the monotone class theorem, it is enough to consider $\Phi\left(\mathfrak{e}_{T_{\mathcal{C}(x, r)}+}\right)$ of form $\Phi_{r, \delta}(\mathfrak{e}):=\Phi\left(\mathfrak{e}_{T_{\mathcal{C}(x, r)}+s}, s \leq T_{\mathcal{C}(x, \delta)} \circ \theta_{T_{\mathcal{C}(x, r)}}\right)$ where $0<\delta<r$ is arbitrary and $\theta$ denotes the usual shift operator. Then

$$
\begin{aligned}
f(b) & =\mathbb{E}_{D}^{b, x}\left[\Phi\left(B_{s}, s \leq T_{\mathcal{C}(x, \delta)}\right)\right] \\
& =\mathbb{E}^{b}\left[\frac{G_{D}\left(x, B_{T_{\mathcal{C}(x, \delta)}}\right)}{G_{D}(x, b)} \Phi\left(B_{s}, s \leq T_{\mathcal{C}(x, \delta)}\right), T_{\mathcal{C}(x, \delta)}<T_{\partial D}\right]
\end{aligned}
$$

where the second equality is due to the $h$-transform. By the definition of $\mu_{D}(x, x ; t)$ and the strong Markov property at $T_{\mathcal{C}(x, \delta)} \circ \theta_{T_{\mathcal{C}(x, r)}}$,

$$
\begin{aligned}
& \mu_{D}(x, x ; t)\left[\Phi_{r, \delta}(\mathfrak{e}), T_{\mathcal{C}(x, r)}<T_{x}\right] \\
& =\lim _{\varepsilon \rightarrow 0^{+}} \frac{1}{\pi \varepsilon^{2}} \mathbb{E}^{x}\left[\Phi_{r, \delta}(B), T_{\mathcal{C}(x, \delta)} \circ \theta_{T_{\mathcal{C}(x, r)}}<t \wedge T_{\partial D}, \mathbb{P}^{a}\left(\left|B_{s}-x\right|<\varepsilon, s<T_{\partial D}\right)\right] \\
& =\mathbb{E}^{x}\left[\Phi_{r, \delta}(B) p_{D}(s, x, a), T_{\mathcal{C}(x, \delta)} \circ \theta_{T_{\mathcal{C}(x, r)}}<t \wedge T_{\partial D}\right],
\end{aligned}
$$


where $s:=t-T_{\mathcal{C}(x, \delta)} \circ \theta_{T_{\mathcal{C}(x, r)}}, a:=B_{T_{\mathcal{C}(x, \delta)} \circ \theta_{T_{\mathcal{C}(x, r)}}}$ and the last equality follows from the definition of the transition probabilities $p_{D}$. Taking the integral over $t$ gives that

$$
\begin{aligned}
& \nu_{D}(x, x)\left[\Phi_{r, \delta}(\mathfrak{e}), T_{\mathcal{C}(x, r)}<T_{x}\right] \\
= & \mathbb{E}^{x}\left[\Phi_{r, \delta}(B) G_{D}\left(x, B_{T_{\mathcal{C}(x, \delta)} \circ \theta_{T_{\mathcal{C}(x, r)}}}\right), T_{\mathcal{C}(x, \delta)} \circ \theta_{T_{\mathcal{C}(x, r)}}<T_{\partial D}\right] .
\end{aligned}
$$

By the strong Markov property at $T_{\mathcal{C}(x, r)}$ and the $h$-transform, the above expectation term $\mathbb{E}^{x}[\cdots]$ is equal to

$$
\mathbb{E}^{x}\left[G_{D}\left(x, B_{T_{\mathcal{C}(x, r)}}\right) f\left(B_{T_{\mathcal{C}(x, r)}}\right), T_{\mathcal{C}(x, r)}<T_{\partial D}\right],
$$

yielding (A.4) because $T_{\mathcal{C}(x, r)}<T_{\partial D}$ holds with probability one.

Acknowledgements. We are grateful to Chris Burdzy for raising the question about existence of local times for thick points. The project was partly supported by ANR MALIN (ANR-16-CE93-0003); E.A. acknowledges supports from ANR GRAAL (ANR14-CE25-0014) and ANR Liouville (ANR-15-CE40-0013), Y.H. acknowledges supports from ANR SWiWS (ANR-17-CE40-0032).

\section{References}

[1] Bass, R.F., Burdzy, K. and Khoshnevisan, D. (1994). Intersection local time for points of infinite multiplicity. Ann. Probab. 22 no. 2, 566-625.

[2] Biggins, J.D. (1977). Martingale convergence in the branching random walk. J. Appl. Probab. 14, 25-37.

[3] Burdzy, K. (1987). Multidimensional Brownian Excursions and Potential Theory. Pitman Research Notes in Mathematics Series, 164 Longman Scientific \& Technical, Harlow; John Wiley \& Sons, Inc., New York.

[4] Cammarota, V. and Mörters, P. (2012). On the most visited sites of planar Brownian motion. Electron. Commun. Probab. 17, no. 15, 1-9.

[5] Dembo, A., Peres, Y., Rosen, J. and Zeitouni, O. (2001). Thick points for planar Brownian motion and the Erdős-Taylor conjecture on random walk. Acta Math. 186, no. 2, 239-270.

[6] Durrett, R. (2010). Probability: Theory and Examples. Fourth edition. Cambridge University Press, Cambridge.

[7] Dvoretzky, A., Erdős, P. and Kakutani, S. (1958). Points of multiplicity $c$ of plane Brownian paths. Bull. Res. Council Israel Sect. F 7, 175-180. 
[8] Jego, A. (2020). Planar Brownian motion and Gaussian multiplicative chaos. Ann. Probab., 48, 1597-1643.

[9] Jego, A. (2019). Characterisation of planar Brownian multiplicative chaos. arXiv:1909.0506\%.

[10] Lawler, G.F. (2005). Conformally Invariant Processes in the Plane. Mathematical Surveys and Monographs, 114. American Mathematical Society, Providence, RI.

[11] Lawler, G.F. and Werner, W. (2004). The Brownian loop soup. Probab. Theory Related Fields 128 no. 4, 565-588.

[12] Le Gall, J.-F. (1985). Sur la mesure de Hausdorff de la courbe brownienne. In: Azéma J., Yor M. (eds) Séminaire de Probabilités XIX 1983/84. Lecture Notes in Mathematics, vol 1123. pp. 297-313. Springer, Berlin, Heidelberg.

[13] Le Gall, J.-F. (1987). Le comportement du mouvement brownien entre les deux instants où il passe par un point double. J. Funct. Anal. 71, no. 2, 246-262.

[14] Le Gall, J.-F. (1990). Some Properties of Planar Brownian Motion. Saint-Flour probability school 1990, Lecture Notes in Mathematics 1527, Springer, Berlin, 1992.

[15] Lyons, R. (1997). A simple path to Biggins' martingale convergence for branching random walk. In: Classical and Modern Branching Processes (Eds.: K.B. Athreya and P. Jagers). IMA Volumes in Mathematics and its Applications 84, 217-221. Springer, New York.

[16] Lyons, R., Pemantle, R. and Peres, Y. (1995). Conceptual proofs of $L \ln L$ criteria for mean behavior of branching processes. Ann. Probab. 23, 1125-1138.

[17] Petrov, V. V. (1995). Limit theorems of probability theory. Sequences of independent random variables. Oxford Studies in Probability, 4. Oxford Science Publications. The Clarendon Press, Oxford University Press, New York.

[18] Pitman, J.W. and Yor, M. (1996). Decomposition at the maximum for excursions and bridges of one-dimensional diffusions. Itô's stochastic calculus and probability theory pp. 293-310, Springer, Tokyo.

[19] Ray, D. (1963). Sojourn times and the exact Hausdorff measure of the sample path for planar Brownian motion. Trans. Amer. Math. Soc. 106, 436-444.

[20] Revuz, D. and Yor, M. (1999). Continuous Martingales and Brownian Motion. Third Edition. Springer-Verlag, Berlin.

[21] Taylor, S.J. (1986). The measure theory of random fractals. Math. Proc. Cambridge Phil. Soc. 100, 383-406. 